Supporting Information

\title{
Full-Color, Narrowband, and High-Efficiency Electroluminescence from Boron and Carbazole Embedded Polycyclic Heteroaromatics
}

Minlang Yang, In Seob Park, and Takuma Yasuda*

INAMORI Frontier Research Center (IFRC) and Department of Applied Chemistry, Graduate School of Engineering, Kyushu University, 744 Motooka, Nishi-ku, Fukuoka 819-0395, Japan

*E-mail: yasuda@ifrc.kyushu-u.ac.jp

\section{Table of contents}

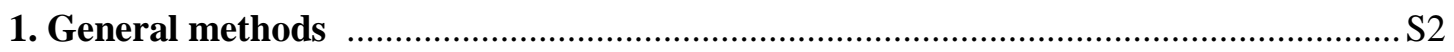

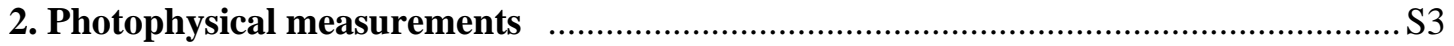

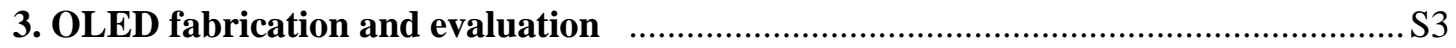

4. Synthesis and characterization

Figures S1-S12. ${ }^{1} \mathrm{H}$ and ${ }^{13} \mathrm{C}\left\{{ }^{1} \mathrm{H}\right\}$ NMR spectra of 1-12 ......................................S10-S20

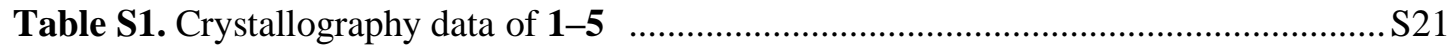

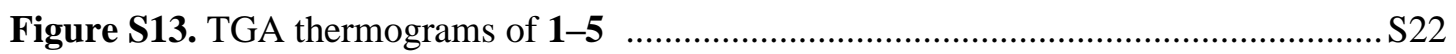

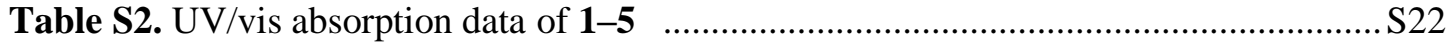

Figures S14-S18. DFT calculations and simulated absorption spectra of 1-5 ..........S22-S24

Figure S19. Fluorescence and phosphorescence spectra of 1-5 in solutions .................... S25

Figure S20. PL spectra and transient PL decay curves of 1-5 in doped films .................. S25

Table S3. Photophysical data of 1-5 in doped films .................................................... S25

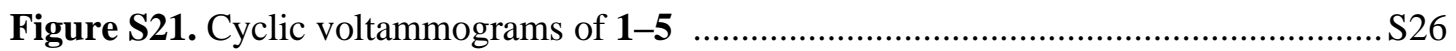

Figure S22. Photoelectron yield spectra of 1-5 in neat films ........................................ S27

Figure S23. Angle-dependent p-polarized PL characteristics of 1-5 in doped films ......... S27

Figure S24. Energy-level diagram of OLEDs (devices A-E) …...................................... S28

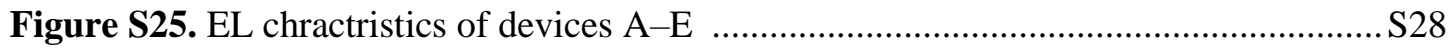

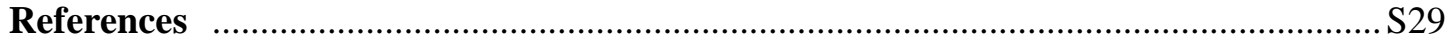

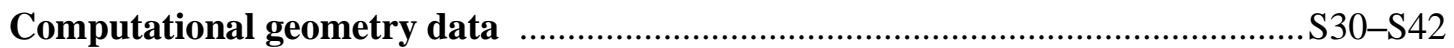




\section{General methods}

All regents and anhydrous solvents were purchased from Sigma-Aldrich, Tokyo Chemical Industry (TCI), or Fujifilm Wako Pure Chemical Corp., and were used without further purification unless otherwise noted. 2,8-Di-tert-butyl-5,11-dihydroindolo[3,2-b]carbazole ${ }^{1}$ and 2,8bis(diphenylphosphoryl)dibenzo[ $[b, d]$ furan $(\mathrm{PPF})^{2}$ were synthesized according to the literature procedures. 2,3,6,7,10,11-Hexacyano-1,4,5,8,9,12-hexaazatriphenylene (HAT-CN) was generously provided by Nippon Soda Co., Ltd. and purified by vacuum sublimation before use. Other OLED materials were purchased from e-Ray Optoelectronics Technology Co., Ltd. and used without further purification.

${ }^{1} \mathrm{H}$ and ${ }^{13} \mathrm{C}\left\{{ }^{1} \mathrm{H}\right\}$ NMR spectra were recorded on a Bruker Avance III 400 spectrometer. Chemical shifts of ${ }^{1} \mathrm{H}$ and ${ }^{13} \mathrm{C}\left\{{ }^{1} \mathrm{H}\right\}$ NMR signals were quoted to tetramethylsilane $(\delta=0.00)$ and $\mathrm{CDCl}_{3}(\delta=77.0)$ as internal standards, respectively. Matrix-assisted laser desorption ionization time-of-flight (MALDI-TOF) mass spectra were collected on a Bruker Daltonics Autoflex III spectrometer using dithranol as the matrix. Elemental analysis was carried out with a Yanaco MT$5 \mathrm{CHN}$ corder. The final products were purified by temperature-gradient vacuum sublimation using an ALS P-100 system before the measurements and device fabrication. Thermogravimetric analysis (TGA) were performed using a Hitachi High-Tech Science TG/DTA7300 analyzer under a $\mathrm{N}_{2}$ atmosphere. Cyclic voltammetry was performed using an ALS CHI 612E electrochemical analyzer and a three-electrode cell equipped with Pt working and counter electrodes and an $\mathrm{Ag} / \mathrm{Ag}^{+}$reference electrode, and the redox potentials were calibrated with ferrocene as an internal standard.

Single crystals suitable for the crystallographic analysis were grown from solutions of chlorobenzene/methanol for $\mathbf{1}$, chloroform/methanol for $\mathbf{2}, \mathbf{4}$, and $\mathbf{5}$, and 1,2dichloroethane/methanol for 3. Single-crystal X-ray diffraction measurements were performed on a Rigaku AFC-10 diffractometer with a Saturn724+ CCD detector. The diffraction data were collected using Mo- $K \alpha$ radiation $(\lambda=0.71073 \AA)$ monochromated with multi-layer mirror. The structures were solved by direct methods (SIR-2014) and refined by the full-matrix least-squares on $F^{2}$ (SHELXL-2018). All non-hydrogen atoms were refined anisotropically, and all hydrogen atoms were placed using AFIX instructions. The crystallographic data have been deposited in the Cambridge Crystallographic Data Centre (CCDC) as supplementary publication materials (CCDC numbers: 2032784-2032788 for compounds 1-5, respectively). These data can be obtained free of charge from the CCDC via www.ccdc.cam.ac.uk/data_request/cif.

Density functional theory (DFT) calculations were performed using the ADF2018 program package. ${ }^{3}$ The molecular geometries in the ground state were optimized using the PBE functional and the DZP basis set in the gas phase. The computational geometry data of 1-5 were appended to the end of the Supporting Information (S30-S42). 


\section{Photophysical measurements}

Organic thin films for photophysical measurements were deposited on quartz or $\mathrm{Si}(100)$ substrates via vacuum deposition under high vacuum $\left(<7 \times 10^{-5} \mathrm{~Pa}\right)$ using an ALS E-200 vacuum evaporation system. UV-vis absorption and photoluminescence (PL) spectra were measured using a Jasco V-670 spectrometer and a Jasco FP-8600 spectrophotometer, respectively. The absolute PL quantum yields $\left(\Phi_{\mathrm{PL}}\right)$ were determined using a Jasco ILF-835 integrating sphere system. The transient PL decay measurements were performed using a Hamamatsu Photonics C11367 Quantaurus-tau fluorescence lifetime spectrometer with an LED excitation source $\left(\lambda_{\mathrm{ex}}=\right.$ $340 \mathrm{~nm}$, pulse width $=100 \mathrm{ps}$, repetition rate $=20 \mathrm{~Hz}$ ) under $\mathrm{N}_{2}$. The ionization potentials (IPs) of materials in neat films were determined using a Riken-Keiki AC-2 ultraviolet photoelectron spectrometer under air. The electron affinities (EAs) were estimated by subtracting the optical energy gap $\left(E_{\mathrm{g}}\right)$ from the measured IP values; $E_{\mathrm{g}}$ values were determined from the high-energy onset position of the PL spectra of the thin films. The photophysical rate constants for radiative decay $\left(k_{\mathrm{r}}\right)$, intersystem crossing $\left(k_{\mathrm{ISC}}\right)$, and reverse intersystem crossing $\left(k_{\mathrm{RISC}}\right)$ were calculated according to the literature method; ${ }^{4} k_{\mathrm{r}}=\Phi_{\mathrm{p}} / \tau_{\mathrm{p}} ; k_{\mathrm{ISC}}=\left(1-\Phi_{\mathrm{p}}\right) / \tau_{\mathrm{p}} ; k_{\mathrm{RISC}}=\Phi_{\mathrm{d}} /\left(k_{\mathrm{ISC}} \cdot \tau_{\mathrm{p}} \cdot \tau_{\mathrm{d}} \cdot \Phi_{\mathrm{p}}\right)$, where $\Phi_{\mathrm{p}}$ and $\Phi_{\mathrm{d}}$ are fractional quantum yields for prompt fluorescence and delayed fluorescence, and $\tau_{\mathrm{p}}$ and $\tau_{\mathrm{d}}$ are emission lifetimes for prompt fluorescence and delayed fluorescence, respectively.

For angle-dependent PL spectroscopy, $2 \mathrm{wt} \%$-emitter:mCBP doped films were vacuumdeposited on quartz substrates and encapsulated with glass plates under $\mathrm{N}_{2}$, which were then attached to an antireflection-coated half cylinder prism using an matching oil. The p-polarized emissions were measured using a Hamamatsu Photonics C13472 molecular orientation measurement system by rotating the stage from $0^{\circ}$ to $90^{\circ}$ under excitation at $340 \mathrm{~nm}$. The angledependent PL spectra were analyzed using Setfos 5.0 software, and the anisotropy factors $(\Theta)$ for the doped films of 1-5 were determined according to the literature method. ${ }^{5}$

\section{OLED fabrication and evaluation}

Indium tin oxide (ITO)-coated glass substrates were cleaned with detergent, deionized water, acetone, and isopropanol. The substrates were then subjected to UV-ozone treatment for $30 \mathrm{~min}$ before being loaded into an ALS E-200 vacuum evaporation system. The organic layers and a cathode aluminum layer were thermally evaporated on the substrates under vacuum $\left(<6 \times 10^{-5}\right.$ Pa) with a deposition rate of $<0.3 \mathrm{~nm} \mathrm{~s}^{-1}$ through a shadow mask, defining a pixel size of 0.04 $\mathrm{cm}^{2}$. The layer thickness and deposition rate were monitored in situ during deposition by an oscillating quartz thickness monitor. OLED characteristics were measured using a Keithley 2400 source meter and a Konica Minolta CS-2000 spectroradiometer. Luminance $(L)$ and external EL quantum efficiency $\left(\eta_{\text {ext }}\right)$ were corrected by Lambertian factors of the devices estimated from the angular dependence of the EL intensity. 


\section{Synthesis and characterization}

All chemical reactions were performed under a dry $\mathrm{N}_{2}$ atmosphere using anhydrous solvents unless otherwise noted.

\section{Synthesis of BBCz-DB (1):}
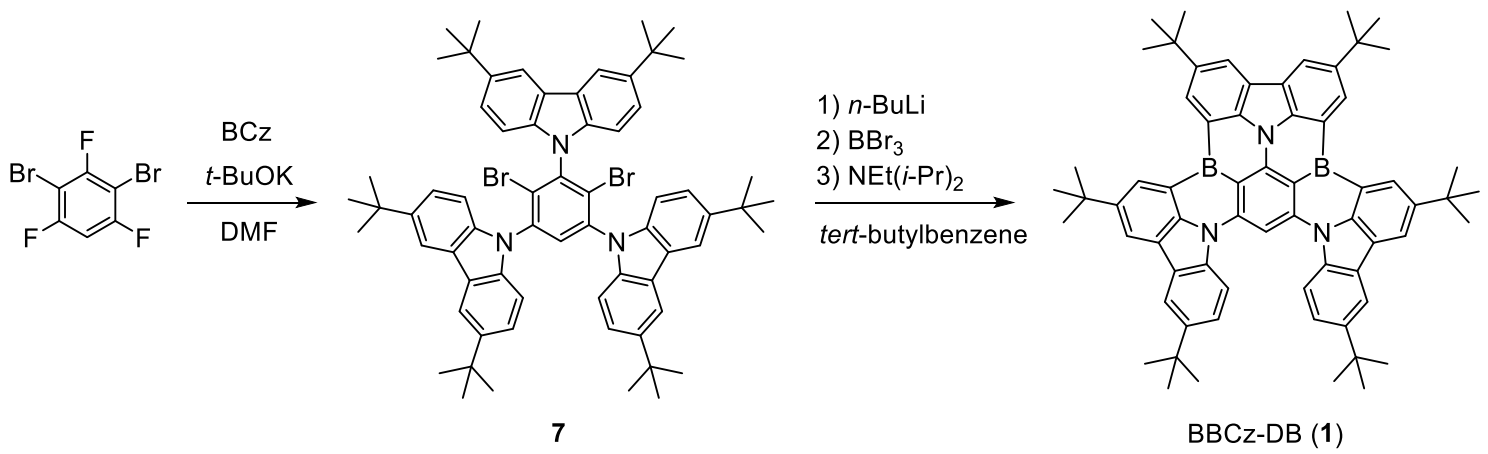

Scheme S1. Synthesis of 1

\section{2,4-Dibromo-1,3,5-tris(3,6-di-tert-butylcarbazol-9-yl)benzene}

(7):

3,6-Di-tertbutylcarbazole $(\mathrm{BCz}, 1.84 \mathrm{~g}, 6.6 \mathrm{mmol})$ and $t$ - BuOK $(0.74 \mathrm{~g}, 6.6 \mathrm{mmol})$ were dissolved in DMF $(60 \mathrm{~mL})$ at room temperature. After stirring for $30 \mathrm{~min}, 2,4$-dibromo-1,3,5-trifluorobenzene $(0.58$ $\mathrm{g}, 2.0 \mathrm{mmol}$ ) was added to the solution. The mixture was stirred at $100{ }^{\circ} \mathrm{C}$ for $12 \mathrm{~h}$. After cooling to room temperature, the reaction mixture was poured into a large amount of water. The product was extracted with chloroform, and the combined organic layer was dried over anhydrous $\mathrm{Na}_{2} \mathrm{SO}_{4}$. After filtration and evaporation, the crude product was purified by recrystallization from chloroform/methanol to afford 7 as a white solid (yield $=1.23 \mathrm{~g}, 58 \%) .{ }^{1} \mathrm{H}$ NMR (400 MHz, $\left.\mathrm{CDCl}_{3}\right): \delta 8.19(\mathrm{~d}, J=1.6 \mathrm{~Hz}, 2 \mathrm{H}), 8.11(\mathrm{~d}, J=2.0 \mathrm{~Hz}, 4 \mathrm{H}), 7.80(\mathrm{~s}, 1 \mathrm{H}), 7.58(\mathrm{dd}, J=8.4,1.6$ $\mathrm{Hz}, 2 \mathrm{H}), 7.52$ (dd, $J=8.8,2.0 \mathrm{~Hz}, 4 \mathrm{H}), 7.18$ (d, $J=8.8 \mathrm{~Hz}, 4 \mathrm{H}), 7.15(\mathrm{~d}, J=6.0 \mathrm{~Hz}, 2 \mathrm{H}), 1.49$ (s, $18 \mathrm{H}), 1.45$ (s, 36H). ${ }^{13} \mathrm{C}\left\{{ }^{1} \mathrm{H}\right\}$ NMR (100 MHz, $\left.\mathrm{CDCl}_{3}\right): \delta 143.67,143.44,140.18,139.26$, 138.80, 137.81, 132.38, 127.87, 124.17, 124.06, 123.71, 123.47, 116.82, 116.63, 109.31, 108.80, 34.86, 34.82, 32.08, 32.01. MS (MALDI-TOF): $\mathrm{m} / z$ calcd $1065.42[\mathrm{M}]^{+}$; found 1065.89.

BBCz-DB (1): To a solution of 7 (2.13 g, $2.0 \mathrm{mmol})$ in tert-butylbenzene was slowly added $n$ $\mathrm{BuLi}(1.6 \mathrm{M}$ in hexane, $3.2 \mathrm{~mL}, 5.0 \mathrm{mmol})$ at $0{ }^{\circ} \mathrm{C}$. After reacting for $4 \mathrm{~h}$ at room temperature, $\mathrm{BBr}_{3}(0.48 \mathrm{~mL}, 5.0 \mathrm{mmol})$ was slowly added at $0{ }^{\circ} \mathrm{C}$, and then the mixture was stirred at room temperature for $12 \mathrm{~h}$. After addition of $\operatorname{NEt}(i-\operatorname{Pr})_{2}(1.3 \mathrm{~mL})$ at $0{ }^{\circ} \mathrm{C}$, the reaction mixture was further stirred at $170^{\circ} \mathrm{C}$ for $24 \mathrm{~h}$. After cooling to room temperature, the reaction mixture was carefully quenched by addition of water. The product was extracted with dichloromethane, and the combined organic layer was dried over anhydrous $\mathrm{Na}_{2} \mathrm{SO}_{4}$. After filtration and evaporation, the crude product was purified by column chromatography on silica gel (eluent: 
hexane/chloroform $=9: 1, \mathrm{v} / \mathrm{v}$ ) and recrystallization from chloroform/methanol to afford $\mathbf{1}$ as a yellow solid (yield $=0.67 \mathrm{~g}, 36 \%) .{ }^{1} \mathrm{H}$ NMR $\left(400 \mathrm{MHz}, \mathrm{CDCl}_{3}\right): \delta 8.71(\mathrm{~s}, 2 \mathrm{H}), 8.60(\mathrm{~s}, 2 \mathrm{H}), 8.33$ (s, 1H), 8.17 (d, $J=1.6 \mathrm{~Hz}, 2 \mathrm{H}), 8.14(\mathrm{~s}, 1 \mathrm{H}), 8.11$ (d, $J=2.0 \mathrm{~Hz}, 2 \mathrm{H}), 7.89$ (d, $J=8.4 \mathrm{~Hz}, 2 \mathrm{H})$, $7.61(\mathrm{dd}, J=8.8,2.0 \mathrm{~Hz}, 2 \mathrm{H}), 1.70(\mathrm{~s}, 18 \mathrm{H}), 1.67(\mathrm{~s}, 18 \mathrm{H}), 1.65(\mathrm{~s}, 18 \mathrm{H}) .{ }^{13} \mathrm{C}\left\{{ }^{1} \mathrm{H}\right\} \mathrm{NMR}(100$ $\left.\mathrm{MHz}, \mathrm{CDCl}_{3}\right): \delta 146.96,144.63,144.48,143.67,142.43,141.60,140.19,137.74,129.10,128.39$, $127.32,124.68,123.82,123.06,122.76,121.29,121.23,119.56,117.11,114.28,114.11,93.21$, 35.23, 35.00, 34.85, 32.33, 32.31, 32.06. MS (MALDI-TOF): $\mathrm{m} / z$ calcd $925.57[\mathrm{M}]^{+}$; found, 925.88. Anal. calcd (\%) for $\mathrm{C}_{66} \mathrm{H}_{69} \mathrm{~B}_{2} \mathrm{~N}_{3}$ : C 85.62, H 7.51, N 4.54; found: C 85.66, H 7.53, N 4.52.

\section{Synthesis of BBCz-SB (2)}
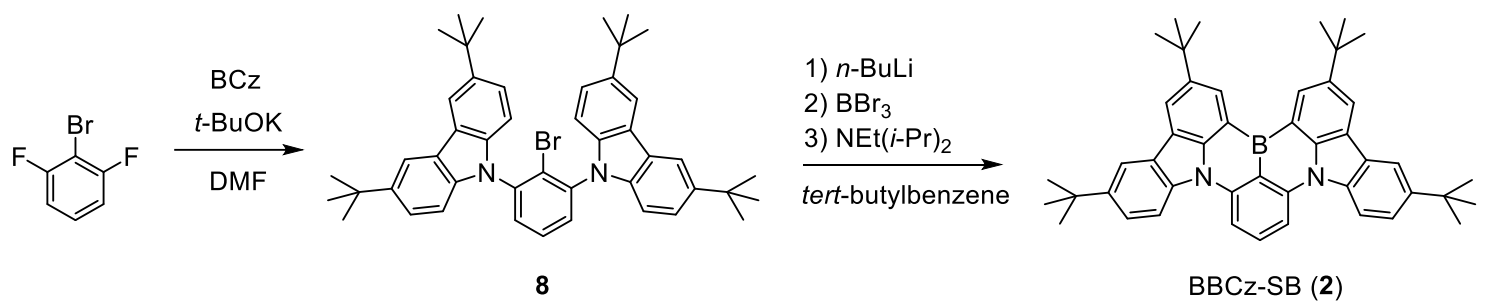

Scheme S2. Synthesis of 2

2-Bromo-1,3-bis(3,6-di-tert-butylcarbazol-9-yl)benzene (8): Compound 8 was synthesized according to the same procedure described above for the synthesis of 7, using 2-bromo-1,3difluorobenzene (1.16 g, $6.0 \mathrm{mmol}), 3,6$-di-tert-butylcarbazole $(\mathrm{BCz}, 4.19 \mathrm{~g}, 15.0 \mathrm{mmol})$, and $t$ $\operatorname{BuOK}(1.68 \mathrm{~g}, 15.0 \mathrm{mmol}$ ), yielding 8 as a white solid (yield $=2.61 \mathrm{~g}, 61 \%) .{ }^{1} \mathrm{H} \mathrm{NMR}(400 \mathrm{MHz}$, $\left.\mathrm{CDCl}_{3}\right): \delta 8.16(\mathrm{~d}, J=2.0 \mathrm{~Hz}, 4 \mathrm{H}), 7.67(\mathrm{dd}, J=9.2,6.0 \mathrm{~Hz}, 1 \mathrm{H}), 7.61(\mathrm{~d}, J=8.0 \mathrm{~Hz}, 2 \mathrm{H}), 7.51$ $(\mathrm{dd}, J=8.4,2.0 \mathrm{~Hz}, 4 \mathrm{H}), 7.11(\mathrm{~d}, J=8.4 \mathrm{~Hz}, 4 \mathrm{H}), 1.47(\mathrm{~s}, 36 \mathrm{H}) \cdot{ }^{13} \mathrm{C}\left\{{ }^{1} \mathrm{H}\right\} \mathrm{NMR}(100 \mathrm{MHz}$, $\left.\mathrm{CDCl}_{3}\right): \delta 143.10,139.67,139.28,130.86,129.24,125.74,123.80,123.39,116.46,109.40,34.80$, 32.07. MS (MALDI-TOF): $\mathrm{m} / z$ calcd $710.32[M]^{+}$; found 710.62 .

BBCz-SB (2): Compound 2 was synthesized according to the same procedure described above for the synthesis of $\mathbf{1}$, using $\mathbf{8}(1.07 \mathrm{~g}, 1.5 \mathrm{mmol}), n$-BuLi (1.6 M, $1.6 \mathrm{~mL}, 2.5 \mathrm{mmol}), \mathrm{BBr}_{3}(0.24$ $\mathrm{mL}, 2.5 \mathrm{mmol}), \mathrm{NEt}(i-\operatorname{Pr})_{2}(0.7 \mathrm{~mL})$, yielding 2 as a yellow solid (yield $\left.=0.23 \mathrm{~g}, 24 \%\right) .{ }^{1} \mathrm{H} \mathrm{NMR}$ (400 MHz, $\left.\mathrm{CDCl}_{3}\right): \delta 9.14(\mathrm{~d}, J=1.6 \mathrm{~Hz}, 2 \mathrm{H}), 8.47(\mathrm{~d}, J=2.0 \mathrm{~Hz}, 2 \mathrm{H}), 8.41(\mathrm{~d}, J=8.8 \mathrm{~Hz}, 2 \mathrm{H})$, $8.35(\mathrm{~d}, J=8.4 \mathrm{~Hz}, 2 \mathrm{H}), 8.27$ (d, $J=2.0 \mathrm{~Hz}, 2 \mathrm{H}), 8.03$ (t, $J=8.0 \mathrm{~Hz}, 1 \mathrm{H}), 7.66$ (dd, $J=8.8,2.2$ $\mathrm{Hz}, 2 \mathrm{H}), 1.67$ (s, $18 \mathrm{H}), 1.54$ (s, $18 \mathrm{H}) .{ }^{13} \mathrm{C}\left\{{ }^{1} \mathrm{H}\right\} \mathrm{NMR}\left(100 \mathrm{MHz}, \mathrm{CDCl}_{3}\right): \delta 145.25,144.58,144.26$, 141.53, 138.35, 132.99, 129.80, 127.05, 124.33, 123.60, 120.63, 117.24, 114.10, 107.93, 35.18, 34.79, 32.20, 31.84. MS (MALDI-TOF): $\mathrm{m} / z$ calcd $640.40[\mathrm{M}]^{+}$; found 640.57. Anal. calcd (\%) for $\mathrm{C}_{46} \mathrm{H}_{49} \mathrm{BN}_{2}$ : C 86.23, H 7.71, N 4.37; found: C 86.41, H 7.78, N 4.37. 


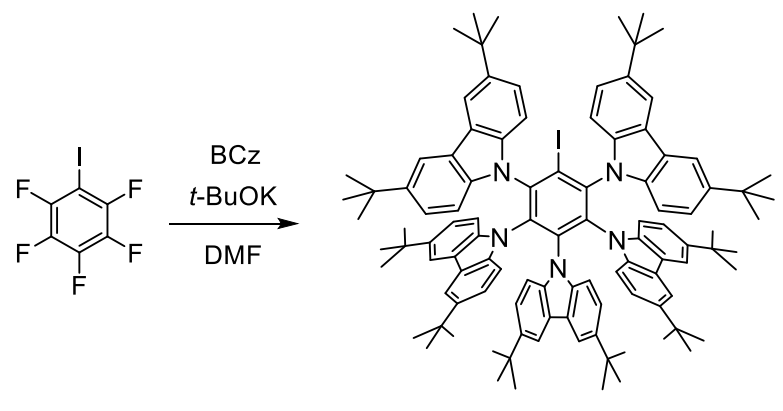

9

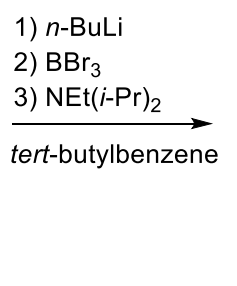

$\underset{\text { tert-butylbenzene }}{\stackrel{\mathrm{NEt}}{ }(i-\mathrm{Pr})_{2}}$

Scheme S3. Synthesis of 3

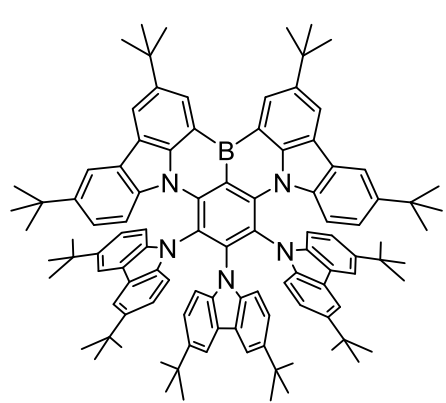

BBCz-G (3)

6-Iodo-1,2,3,4,5-pentakis(3,6-di-tert-butylcarbazol-9-yl)benzene (9): Compound 9 was synthesized according to the same procedure described above for the synthesis of $\mathbf{7}$, using 1,2,3,4,5-pentafluoro-6-iodobenzene (0.88 g, $3.0 \mathrm{mmol}$ ), 3,6-di-tert-butylcarbazole ( $\mathrm{BCz}, 4.61 \mathrm{~g}$, $16.5 \mathrm{mmol}$ ), and $t$-BuOK (1.85 g, $16.5 \mathrm{mmol}$ ), yielding 9 as a white solid (yield $=2.82 \mathrm{~g}, 59 \%$ ). ${ }^{1} \mathrm{H}$ NMR (400 MHz, $\left.\mathrm{CDCl}_{3}\right): \delta 7.60(\mathrm{~d}, J=1.2 \mathrm{~Hz}, 4 \mathrm{H}), 7.21-7.15(\mathrm{~m}, 8 \mathrm{H}), 7.02(\mathrm{dd}, J=8.8,2.0$ $\mathrm{Hz}, 4 \mathrm{H}), 6.93(\mathrm{~d}, J=8.4 \mathrm{~Hz}, 4 \mathrm{H}), 6.81(\mathrm{~d}, J=8.4 \mathrm{~Hz}, 4 \mathrm{H}), 6.67(\mathrm{dd}, J=8.8,2.0 \mathrm{~Hz}, 2 \mathrm{H}), 6.58$ $(\mathrm{dd}, J=8.4,2.0 \mathrm{~Hz}, 4 \mathrm{H}), 1.35$ (s, 36H), 1.22 (s, 36H), $1.12(\mathrm{~s}, 18 \mathrm{H}) .{ }^{13} \mathrm{C}\left\{{ }^{1} \mathrm{H}\right\}$ NMR $(100 \mathrm{MHz}$, $\left.\mathrm{CDCl}_{3}\right): \delta 142.56,142.17,141.30,139.27,137.90,137.54,137.29,137.16,124.08,123.74$, $123.55,122.51,122.36,122.00,121.34,115.50,114.81,114.52,110.71,110.66,110.35,110.25$, 34.46, 34.17, 31.88, 31.76, 31.61. MS (MALDI-TOF): $\mathrm{m} / z$ calcd $1589.86[M]^{+}$; found 1589.80.

BBCz-G (3): Compound 3 was synthesized according to the same procedure described above for the synthesis of $\mathbf{1}$, using $\mathbf{9}$ (3.18 g, $2.0 \mathrm{mmol}), n$-BuLi (1.6 M, $1.9 \mathrm{~mL}, 3.0 \mathrm{mmol}), \mathrm{BBr}_{3}(0.29$ $\mathrm{mL}, 3.0 \mathrm{mmol}), \mathrm{NEt}(i-\operatorname{Pr})_{2}(0.8 \mathrm{~mL})$, yielding 3 as a yellow solid (yield $\left.=0.68 \mathrm{~g}, 23 \%\right) .{ }^{1} \mathrm{H} \mathrm{NMR}$ (400 MHz, $\left.\mathrm{CDCl}_{3}\right): \delta 8.78(\mathrm{~d}, J=2.0 \mathrm{~Hz}, 2 \mathrm{H}), 8.35(\mathrm{~d}, J=1.6 \mathrm{~Hz}, 2 \mathrm{H}), 7.71(\mathrm{~d}, J=2.0 \mathrm{~Hz}, 2 \mathrm{H})$, $7.35(\mathrm{~d}, J=2.0 \mathrm{~Hz}, 4 \mathrm{H}), 7.22(\mathrm{~d}, J=9.2 \mathrm{~Hz}, 2 \mathrm{H}), 7.18(\mathrm{~d}, J=1.6 \mathrm{~Hz}, 2 \mathrm{H}), 6.53(\mathrm{dd}, J=8.6,1.8$ $\mathrm{Hz}, 4 \mathrm{H}), 6.43(\mathrm{~d}, J=8.4 \mathrm{~Hz}, 4 \mathrm{H}), 6.32(\mathrm{dd}, J=8.8,2.0 \mathrm{~Hz}, 2 \mathrm{H}), 6.10(\mathrm{dd}, J=8.8,2.0 \mathrm{~Hz}, 2 \mathrm{H})$, $5.85(\mathrm{~d}, J=8.8 \mathrm{~Hz}, 2 \mathrm{H}), 1.69(\mathrm{~s}, 18 \mathrm{H}), 1.23(\mathrm{~s}, 18 \mathrm{H}), 1.21(\mathrm{~s}, 36 \mathrm{H}), 1.08(\mathrm{~s}, 18 \mathrm{H}) .{ }^{13} \mathrm{C}\left\{{ }^{1} \mathrm{H}\right\} \mathrm{NMR}$ (100 MHz, $\left.\mathrm{CDCl}_{3}\right): \delta 145.85,145.02,143.32,143.27,141.92,141.13,139.63,138.24,137.89$, $137.65,128.85,125.77,125.13,124.24,123.48,122.48,122.23,121.85,121.58,120.94,115.56$, 115.23, 114.94, 114.78, 114.32, 109.51, 109.04, 108.98, 35.26, 34.24, 34.20, 34.18, 32.20, 31.90, 31.76, 31.36. MS (MALDI-TOF): $\mathrm{m} / \mathrm{z}$ calcd $1471.95\left[\mathrm{M}^{+}\right.$; found 1471.37. Anal. calcd (\%) for $\mathrm{C}_{106} \mathrm{H}_{118} \mathrm{BN}_{5}$ : C 86.44, H 8.08, N 4.75; found: C 86.31, H 8.05, N 4.70. 


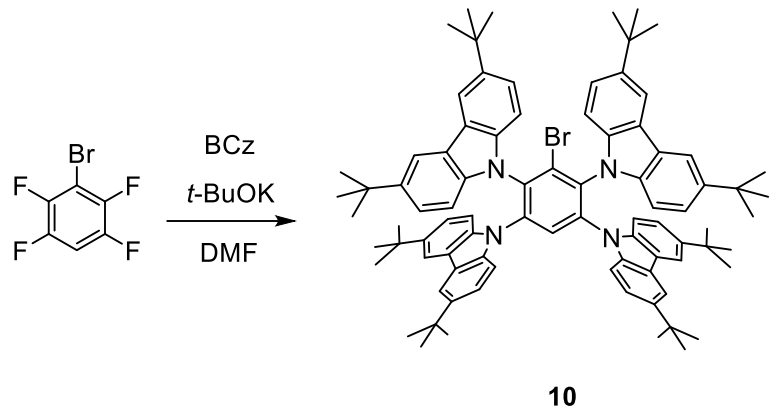

Scheme S4. Synthesis of 4

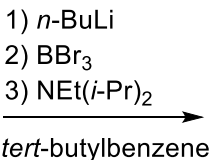

tert-butylbenzene

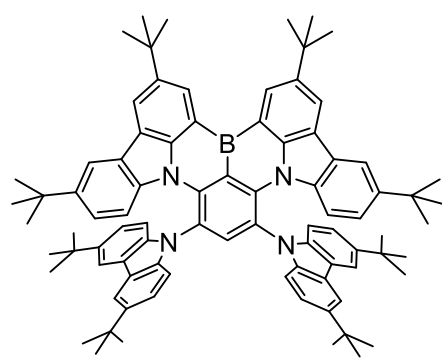

BBCz-Y (4)

3-Bromo-1,2,4,5-tetrakis(3,6-di-tert-butylcarbazol-9-yl)benzene (10): Compound 10 was synthesized according to the same procedure described above for the synthesis of 7 , using 3 bromo-1,2,4,5-tetrafluorobenzene $(0.69 \mathrm{~g}, 3.0 \mathrm{mmol})$, 3,6-di-tert-butylcarbazole $(\mathrm{BCz}, 3.77 \mathrm{~g}$, $13.5 \mathrm{mmol})$, and $t$-BuOK $(1.51 \mathrm{~g}, 13.5 \mathrm{mmol})$. The crude product was purified by column chromatography on silica gel (eluent: hexane/chloroform $=4: 1, \mathrm{v} / \mathrm{v}$ ), followed by recrystallization from chloroform/methanol to afford 10 as a white solid (yield $=2.18 \mathrm{~g}, 57 \%) .{ }^{1} \mathrm{H}$ NMR (400 MHz, $\left.\mathrm{CDCl}_{3}\right): \delta 8.18(\mathrm{~s}, 1 \mathrm{H}), 7.63-7.60(\mathrm{~m}, 8 \mathrm{H}), 7.10-6.95(\mathrm{~m}, 16 \mathrm{H}), 1.38-1.37(\mathrm{~m}, 72 \mathrm{H}) .{ }^{13} \mathrm{C}\left\{{ }^{1} \mathrm{H}\right\}$ NMR (100 MHz, $\left.\mathrm{CDCl}_{3}\right): \delta 143.11,142.92,138.19,137.22,137.18,135.37,131.35,130.50$, 123.88, 123.79, 122.65, 115.53, 115.42, 110.18, 110.08, 109.21, 34.52, 31.93.

BBCz-Y (4): Compound 4 was synthesized according to the same procedure described above for the synthesis of $\mathbf{1}$, using $\mathbf{1 0}(2.53 \mathrm{~g}, 2.0 \mathrm{mmol}), n-\mathrm{BuLi}(1.6 \mathrm{M}, 1.9 \mathrm{~mL}, 3.0 \mathrm{mmol}), \mathrm{BBr}_{3}(0.29$ $\mathrm{mL}, 3.0 \mathrm{mmol})$, and $\mathrm{NEt}(i-\operatorname{Pr})_{2}(0.8 \mathrm{~mL})$, yielding 4 as an orange solid (yield $\left.=0.68 \mathrm{~g}, 28 \%\right) .{ }^{1} \mathrm{H}$ NMR (400 MHz, $\left.\mathrm{CDCl}_{3}\right): \delta 9.22(\mathrm{~d}, J=1.6 \mathrm{~Hz}, 2 \mathrm{H}), 8.36(\mathrm{~d}, J=1.6 \mathrm{~Hz}, 2 \mathrm{H}), 8.25(\mathrm{~s}, 1 \mathrm{H}), 7.92$ $(\mathrm{d}, J=1.6 \mathrm{~Hz}, 4 \mathrm{H}), 7.70(\mathrm{~d}, J=1.6 \mathrm{~Hz}, 2 \mathrm{H}), 7.26(\mathrm{br}, 4 \mathrm{H}), 7.06(\mathrm{br}, 4 \mathrm{H}), 6.72(\mathrm{~d}, J=8.8 \mathrm{~Hz}, 2 \mathrm{H})$, $6.31(\mathrm{dd}, J=8.8,2.0 \mathrm{~Hz}, 2 \mathrm{H}), 1.72(\mathrm{~s}, 18 \mathrm{H}), 1.39(\mathrm{~s}, 36 \mathrm{H}), 1.18(\mathrm{~s}, 18 \mathrm{H}) .{ }^{13} \mathrm{C}\left\{{ }^{1} \mathrm{H}\right\} \mathrm{NMR}(100$ $\left.\mathrm{MHz}, \mathrm{CDCl}_{3}\right): \delta 145.11,144.60,142.79,142.70,138.44,137.92,137.32,136.83,129.28,125.27$, $124.84,124.05,123.49,122.39,121.62,121.38,120.62$, 115.93, 115.11, 113.05, 108.56, 35.29, 34.63, 34.29, 32.22, 31.95, 31.46. MS (MALDI-TOF): $\mathrm{m} / z$ calcd $1194.76\left[\mathrm{M}^{+}\right.$; found 1194.33. Anal. calcd (\%) for $\mathrm{C}_{86} \mathrm{H}_{95} \mathrm{BN}_{4}$ : C 86.40, H 8.01, N 4.69; found: C 86.35, H 8.07, N 4.64. 


\section{Synthesis of BBCz-R (5)}

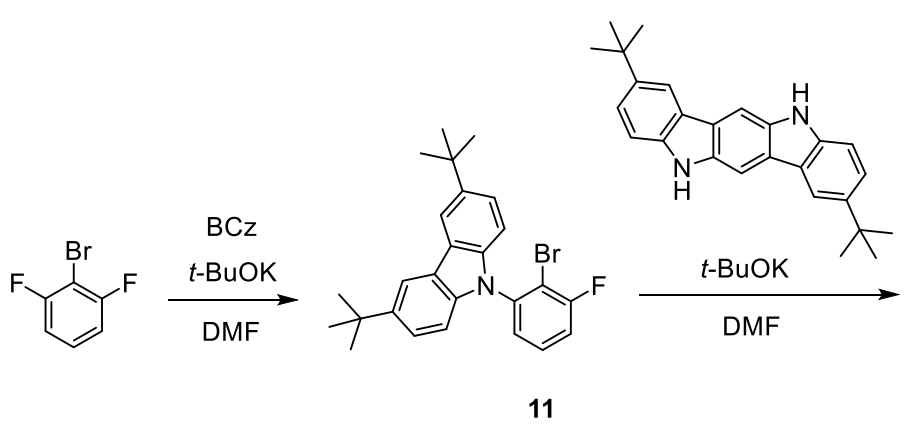

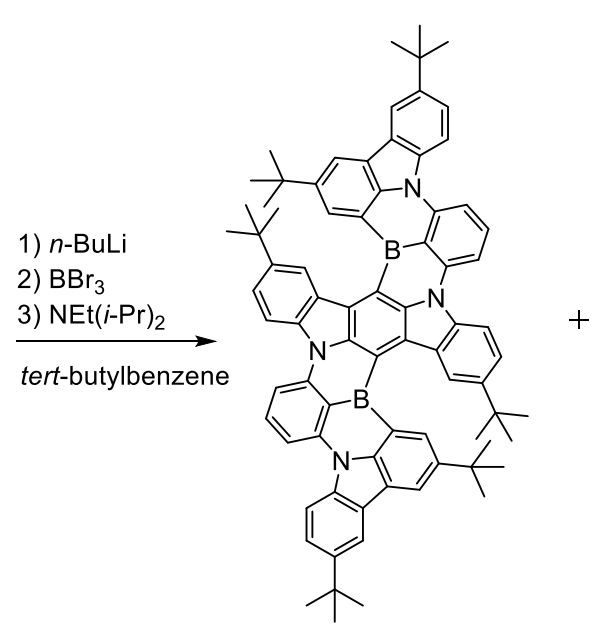

BBCz-R (5)
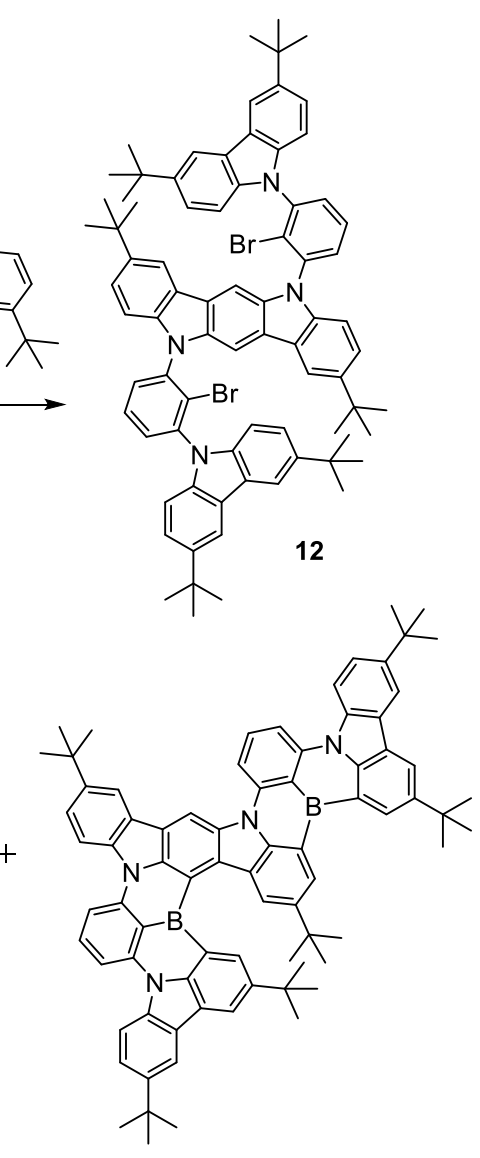

BBCz-Y-II (6)

Scheme S5. Synthesis of 5

2-Bromo-1-fluoro-3-(3,6-di-tert-butylcarbazol-9-yl)benzene (11): Compound 11 was synthesized according to the same procedure described above for the synthesis of 7, using 2bromo-1,3-difluorobenzene $(5.79 \mathrm{~g}, 30.0 \mathrm{mmol})$, 3,6-di-tert-butylcarbazole (BCz, $5.59 \mathrm{~g}, 20.0$ $\mathrm{mmol})$, and $t$-BuOK $(2.24 \mathrm{~g}, 20.0 \mathrm{mmol})$. The product was purified by column chromatography on silica gel (eluent: hexane:chloroform $=4: 1, \mathrm{v} / \mathrm{v}$ ), followed by recrystallization from chloroform/methanol to afford 11 as a white solid (yield $=4.51 \mathrm{~g}, 50 \%$ ). ${ }^{1} \mathrm{H}$ NMR (400 MHz, $\left.\mathrm{CDCl}_{3}\right): \delta 8.14(\mathrm{~d}, J=1.6 \mathrm{~Hz}, 2 \mathrm{H}), 7.46-7.41(\mathrm{~m}, 3 \mathrm{H}), 7.31-7.23(\mathrm{~m}, 2 \mathrm{H}), 6.98(\mathrm{~d}, J=8.8 \mathrm{~Hz}$, 2H), 1.46 (s, 18H). ${ }^{13} \mathrm{C}\left\{{ }^{1} \mathrm{H}\right\}$ NMR $\left(100 \mathrm{MHz}, \mathrm{CDCl}_{3}\right): \delta 143.16,139.42,139.22,129.12,129.04$, 126.32, 123.74, 123.44, 116.43, 116.14, 111.51, 109.53, 34.82, 32.09. MS (MALDI-TOF): $\mathrm{m} / \mathrm{z}$ calcd $451.13[M]^{+}$; found 450.80 .

\section{5,11-Bis(2-bromo-3-(3,6-di-tert-butylcarbazol-9-yl)phenyl)-2,8-di-tert-butyl-indolo[3,2-}

b]carbazole (12): Compound 12 was synthesized using 2,8-di-tert-butyl-5,11-dihydroindolo[3,2$b$ ] carbazole $^{1}(3.69 \mathrm{~g}, 10.0 \mathrm{mmol}), \mathbf{1 1}(11.31 \mathrm{~g}, 25.0 \mathrm{mmol})$, and $t$-BuOK $(2.81 \mathrm{~g}, 25.0 \mathrm{mmol})$. The 
crude product was purified by column chromatography on silica gel (eluent: hexane/chloroform $=3: 1, \mathrm{v} / \mathrm{v}$ ), followed by recrystallization from chloroform/methanol to afford $\mathbf{1 2}$ as a white solid (yield $=4.82 \mathrm{~g}, 39 \%) .{ }^{1} \mathrm{H}$ NMR $\left(400 \mathrm{MHz}, \mathrm{CDCl}_{3}\right): \delta 8.22-8.18(\mathrm{~m}, 6 \mathrm{H}), 7.85(\mathrm{~s}, 2 \mathrm{H}), 7.78-7.69$ (m, 6H), 7.60-7.51 (m, 6H), $7.32(\mathrm{~d}, J=8.8 \mathrm{~Hz}, 2 \mathrm{H}), 7.20(\mathrm{dd}, J=8.4,6.4 \mathrm{~Hz}, 4 \mathrm{H}), 1.52(\mathrm{~s}, 18 \mathrm{H})$, $1.49(\mathrm{~d}, J=2.0 \mathrm{~Hz}, 36 \mathrm{H}) .{ }^{13} \mathrm{C}\left\{{ }^{1} \mathrm{H}\right\} \mathrm{NMR}\left(100 \mathrm{MHz}, \mathrm{CDCl}_{3}\right): \delta 143.11,142.76,140.00,139.78$, 139.21, 139.13, 137.19, 130.91, 130.65, 129.44, 125.73, 124.11, 123.77, 123.67, 123.40, 123.19, 116.47, 109.53, 109.40, 109.31, 100.17, 34.75, 32.03, 31.99 .

BBCz-R (5): Compound 5 was synthesized according to the same procedure described above for the synthesis of $\mathbf{1}$, using 12 (1.11 g, $0.90 \mathrm{mmol}), n$-BuLi (1.6 M, $1.7 \mathrm{~mL}, 2.7 \mathrm{mmol}), \mathrm{BBr}_{3}(0.3$ $\mathrm{mL}, 2.7 \mathrm{~mol}), \mathrm{NEt}(i-\operatorname{Pr})_{2}(0.7 \mathrm{~mL})$, yielding 5 as a black solid (yield $\left.=0.054 \mathrm{~g}, 5 \%\right) .{ }^{1} \mathrm{H} \mathrm{NMR}$ $\left(400 \mathrm{MHz}, \mathrm{CDCl}_{3}\right): \delta 8.67(\mathrm{~d}, J=1.6 \mathrm{~Hz}, 2 \mathrm{H}), 8.46(\mathrm{~d}, J=8.8 \mathrm{~Hz}, 2 \mathrm{H}), 8.42(\mathrm{~d}, J=2.0 \mathrm{~Hz}, 2 \mathrm{H})$, $8.41-8.31(\mathrm{~m}, 10 \mathrm{H}), 8.10(\mathrm{t}, J=8.2 \mathrm{~Hz}, 2 \mathrm{H}), 7.74(\mathrm{dd}, J=8.6,2.0 \mathrm{~Hz}, 2 \mathrm{H}), 7.51(\mathrm{dd}, J=8.4,1.6$ $\mathrm{Hz}, 2 \mathrm{H}), 1.58(\mathrm{~s}, 18 \mathrm{H}), 1.32(\mathrm{~s}, 18 \mathrm{H}), 1.09(\mathrm{~s}, 18 \mathrm{H}) \cdot{ }^{13} \mathrm{C}\left\{{ }^{1} \mathrm{H}\right\} \mathrm{NMR}$ spectrum of 5 was not obtained because of its low solubility in $\mathrm{CDCl}_{3}$. MS (MALDI-TOF): $\mathrm{m} / \mathrm{z}$ calcd $1090.63[\mathrm{M}]^{+}$; found 1089.90 . Anal. calcd (\%) for $\mathrm{C}_{78} \mathrm{H}_{76} \mathrm{~B}_{2} \mathrm{~N}_{4}$ : C 85.86, H 7.02, N 5.13; found: C 85.85, H 6.96, N 5.11.

BBCz-Y-II (6): Compound 6, an asymmetrical isomer of $\mathbf{5}$, was simultaneously obtained as a byproduct during the synthesis of $\mathbf{5}$. This compound was separated by column chromatography and obtained as an orange solid (yield $=0.065 \mathrm{~g}, 7 \%)$. ${ }^{1} \mathrm{H} \mathrm{NMR}\left(400 \mathrm{MHz}, \mathrm{CDCl}_{3}\right): \delta 9.52$ (s, $1 \mathrm{H}), 9.14(\mathrm{dd}, J=10.8,2.0 \mathrm{~Hz}, 2 \mathrm{H}), 8.75(\mathrm{~d}, J=2.0 \mathrm{~Hz}, 1 \mathrm{H}), 8.70(\mathrm{~d}, J=8.0 \mathrm{~Hz}, 1 \mathrm{H}), 8.63$ (d, $J$ $=2.0 \mathrm{~Hz}, 1 \mathrm{H}), 8.50-8.39(\mathrm{~m}, 8 \mathrm{H}), 8.33-8.29(\mathrm{~m}, 3 \mathrm{H}), 8.24(\mathrm{~d}, J=8.0 \mathrm{~Hz}, 1 \mathrm{H}), 8.07(\mathrm{t}, J=8.0 \mathrm{~Hz}$, $1 \mathrm{H}), 7.76-7.69(\mathrm{~m}, 3 \mathrm{H}), 1.68(\mathrm{~s}, 9 \mathrm{H}), 1.60(\mathrm{~s}, 9 \mathrm{H}), 1.58(\mathrm{~s}, 9 \mathrm{H}), 1.56(\mathrm{~s}, 9 \mathrm{H}), 1.26(\mathrm{~s}, 18 \mathrm{H}) .{ }^{13} \mathrm{C}\left\{{ }^{1} \mathrm{H}\right\}$ NMR spectrum of 6 was not obtained because of its low solubility in $\mathrm{CDCl}_{3}$. MS (MALDI-TOF): $m / z$ calcd 1090.63 [M] $]^{+}$; found 1090.10. Anal. calcd (\%) for $\mathrm{C}_{78} \mathrm{H}_{76} \mathrm{~B}_{2} \mathrm{~N}_{4}$ : C 85.86, H 7.02, N 5.13; found: C 85.83, H 6.98, N 5.13. 

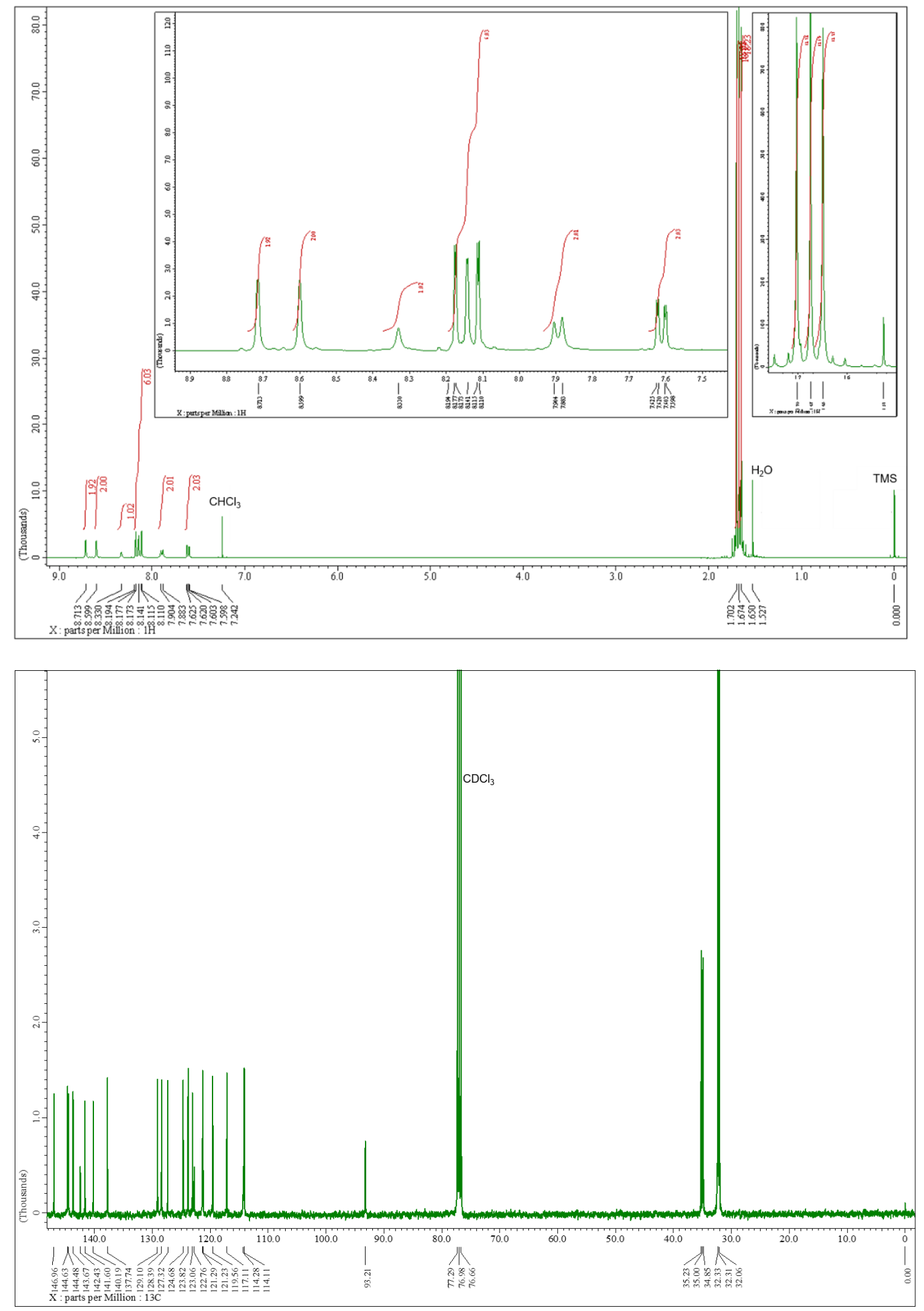

Figure S1. ${ }^{1} \mathrm{H}$ and ${ }^{13} \mathrm{C}\left\{{ }^{1} \mathrm{H}\right\}$ NMR spectra of $\mathbf{1}$ in $\mathrm{CDCl}_{3}$. 

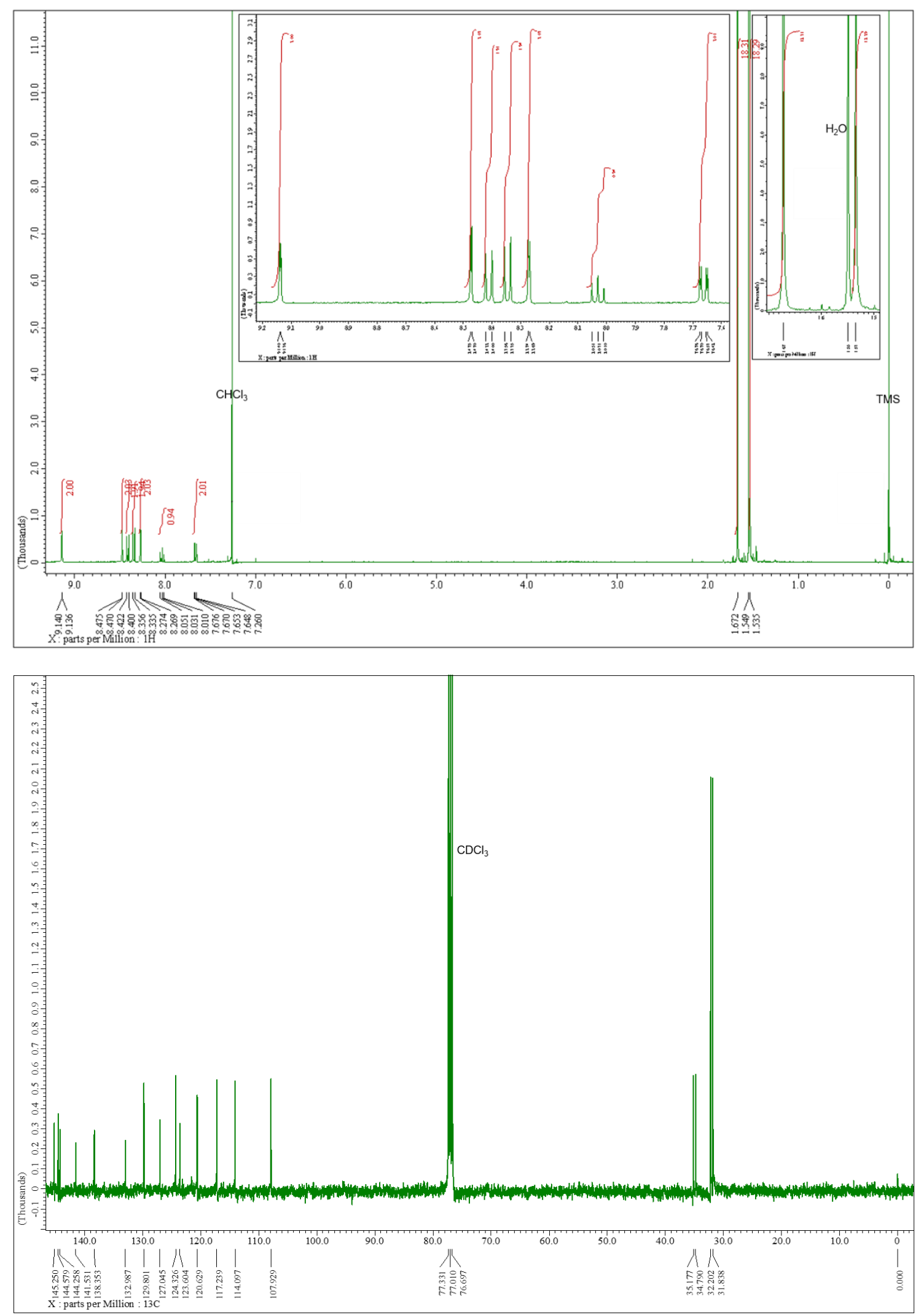

Figure S2. ${ }^{1} \mathrm{H}$ and ${ }^{13} \mathrm{C}\left\{{ }^{1} \mathrm{H}\right\}$ NMR spectra of $\mathbf{2}$ in $\mathrm{CDCl}_{3}$. 

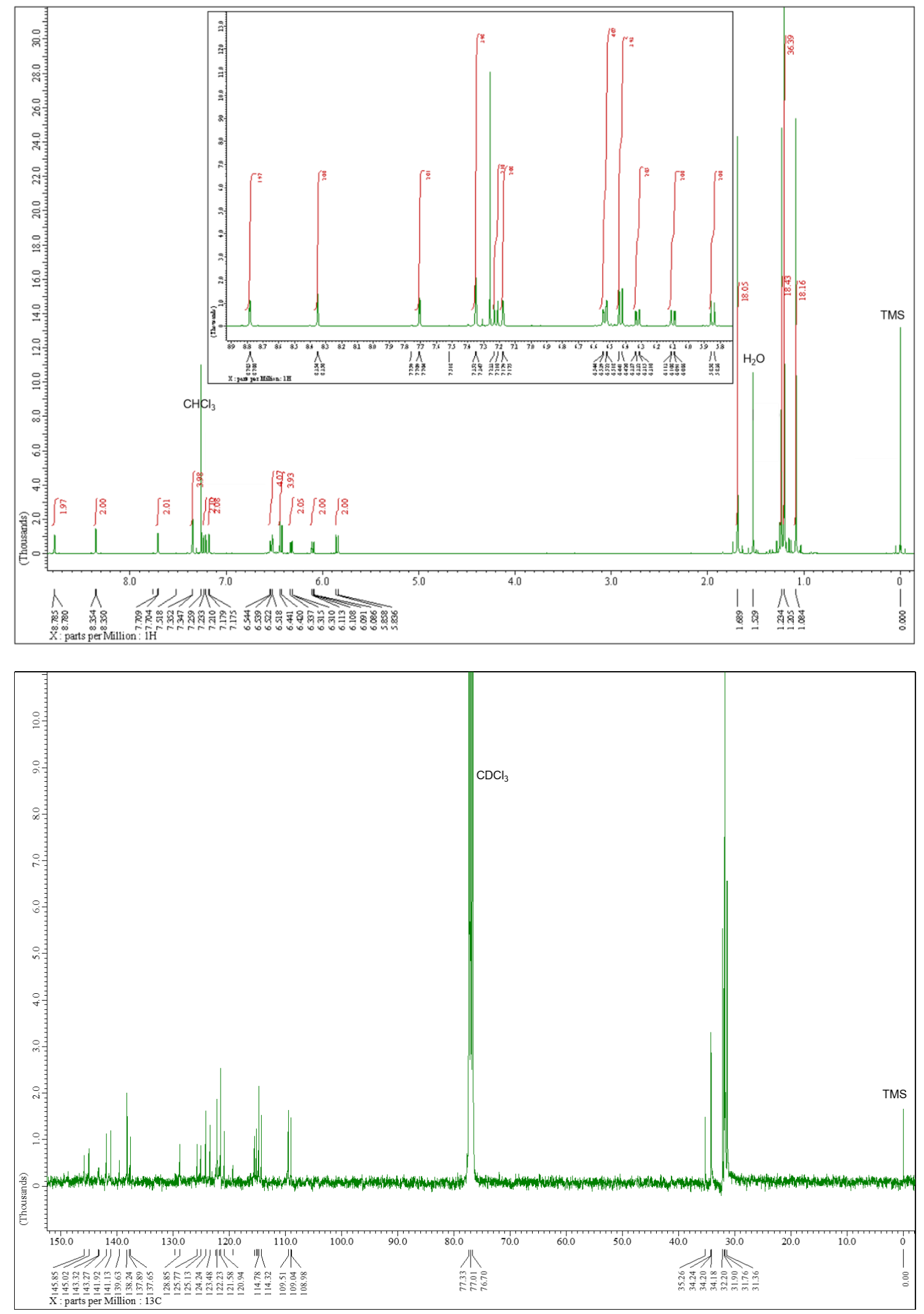

Figure S3. ${ }^{1} \mathrm{H}$ and ${ }^{13} \mathrm{C}\left\{{ }^{1} \mathrm{H}\right\}$ NMR spectra of $\mathbf{3}$ in $\mathrm{CDCl}_{3}$. 

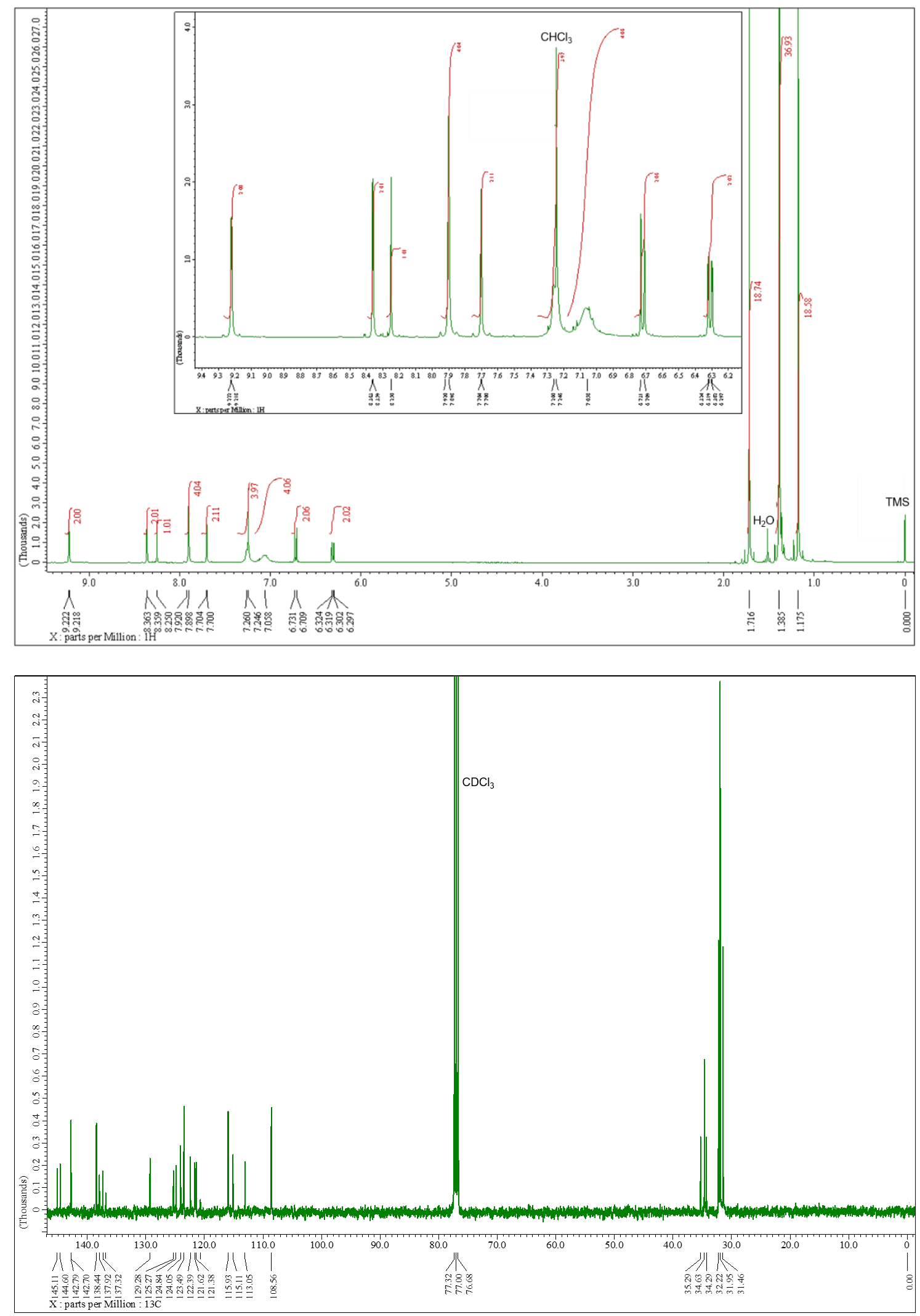

Figure S4. ${ }^{1} \mathrm{H}$ and ${ }^{13} \mathrm{C}\left\{{ }^{1} \mathrm{H}\right\}$ NMR spectra of 4 in $\mathrm{CDCl}_{3}$. 


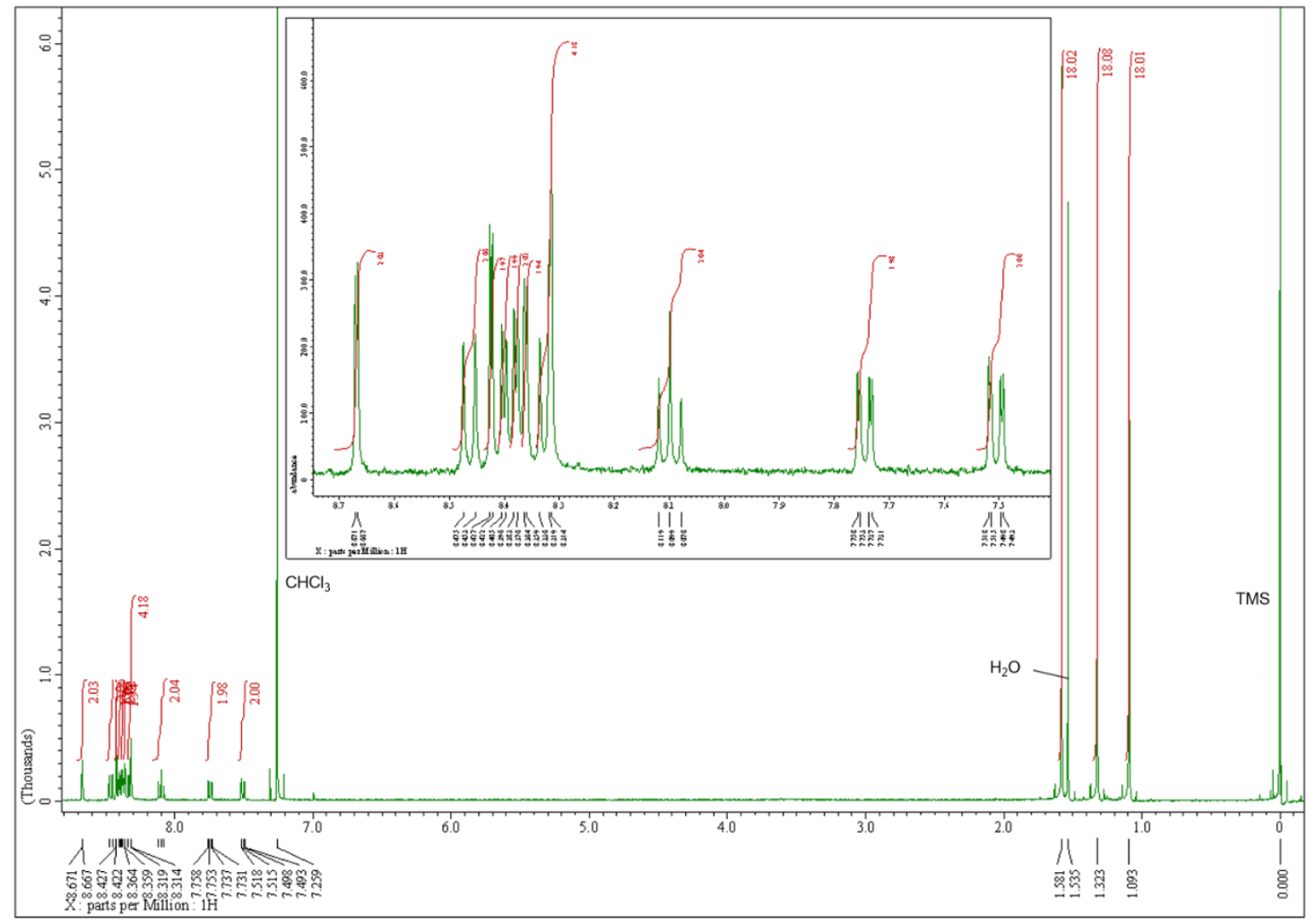

Figure S5. ${ }^{1} \mathrm{H}$ NMR spectrum of 5 in $\mathrm{CDCl}_{3}$.

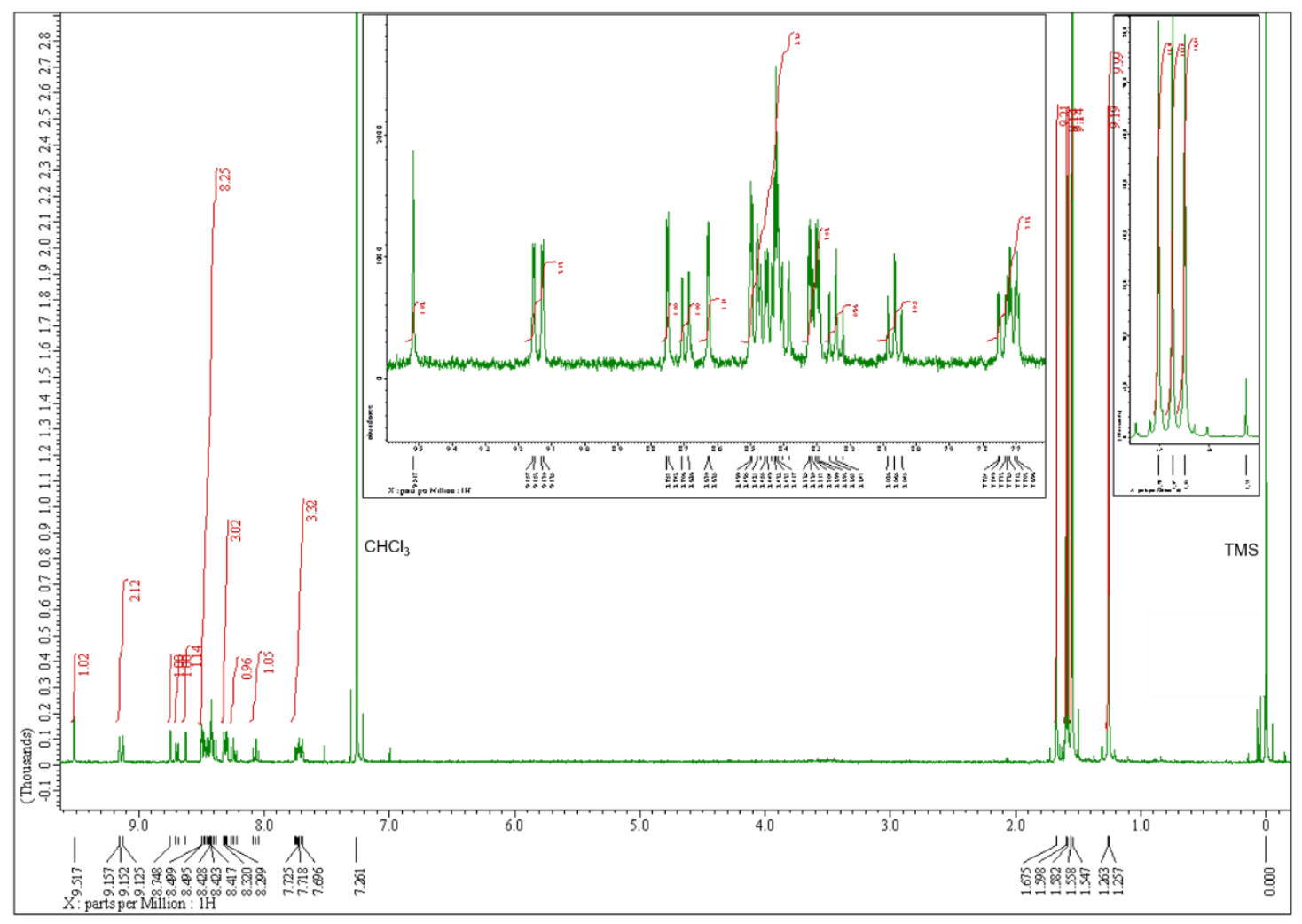

Figure S6. ${ }^{1} \mathrm{H}$ NMR spectrum of 6 in $\mathrm{CDCl}_{3}$. 

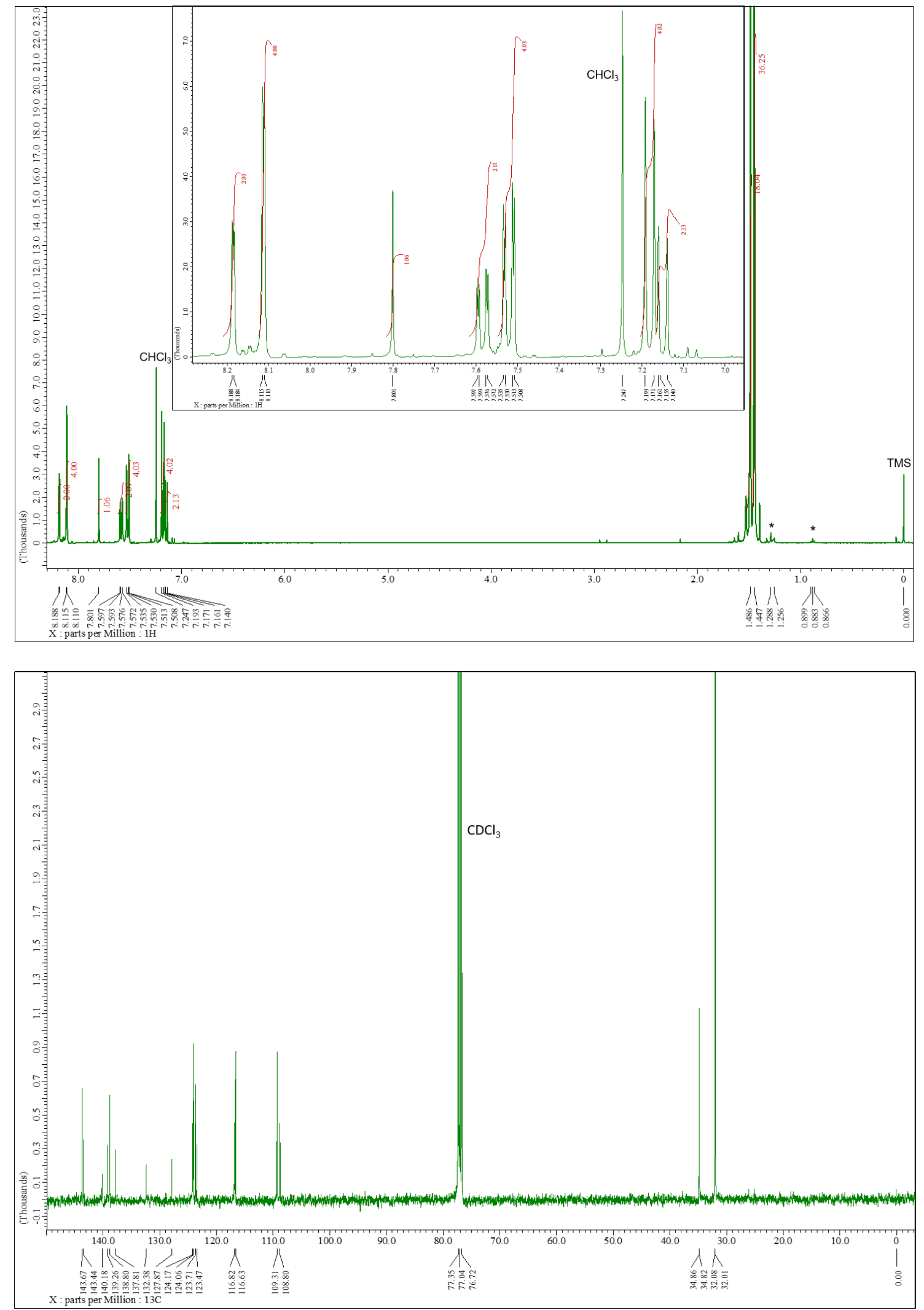

Figure S7. ${ }^{1} \mathrm{H}$ and ${ }^{13} \mathrm{C}\left\{{ }^{1} \mathrm{H}\right\}$ NMR spectra of 7 in $\mathrm{CDCl}_{3}$. ${ }^{*}$ Solvent impurities. 

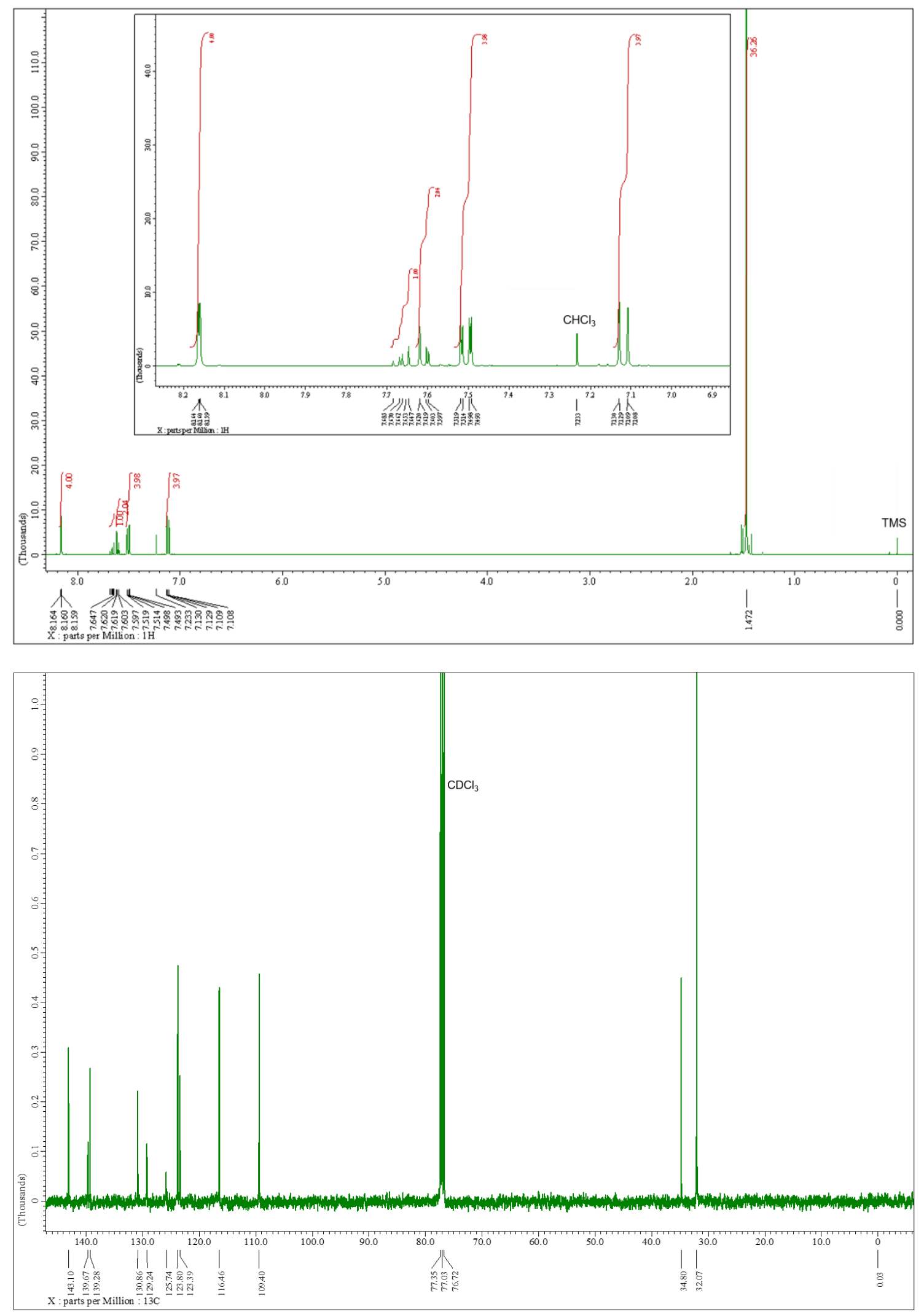

Figure S8. ${ }^{1} \mathrm{H}$ and ${ }^{13} \mathrm{C}\left\{{ }^{1} \mathrm{H}\right\}$ NMR spectra of 8 in $\mathrm{CDCl}_{3}$. 

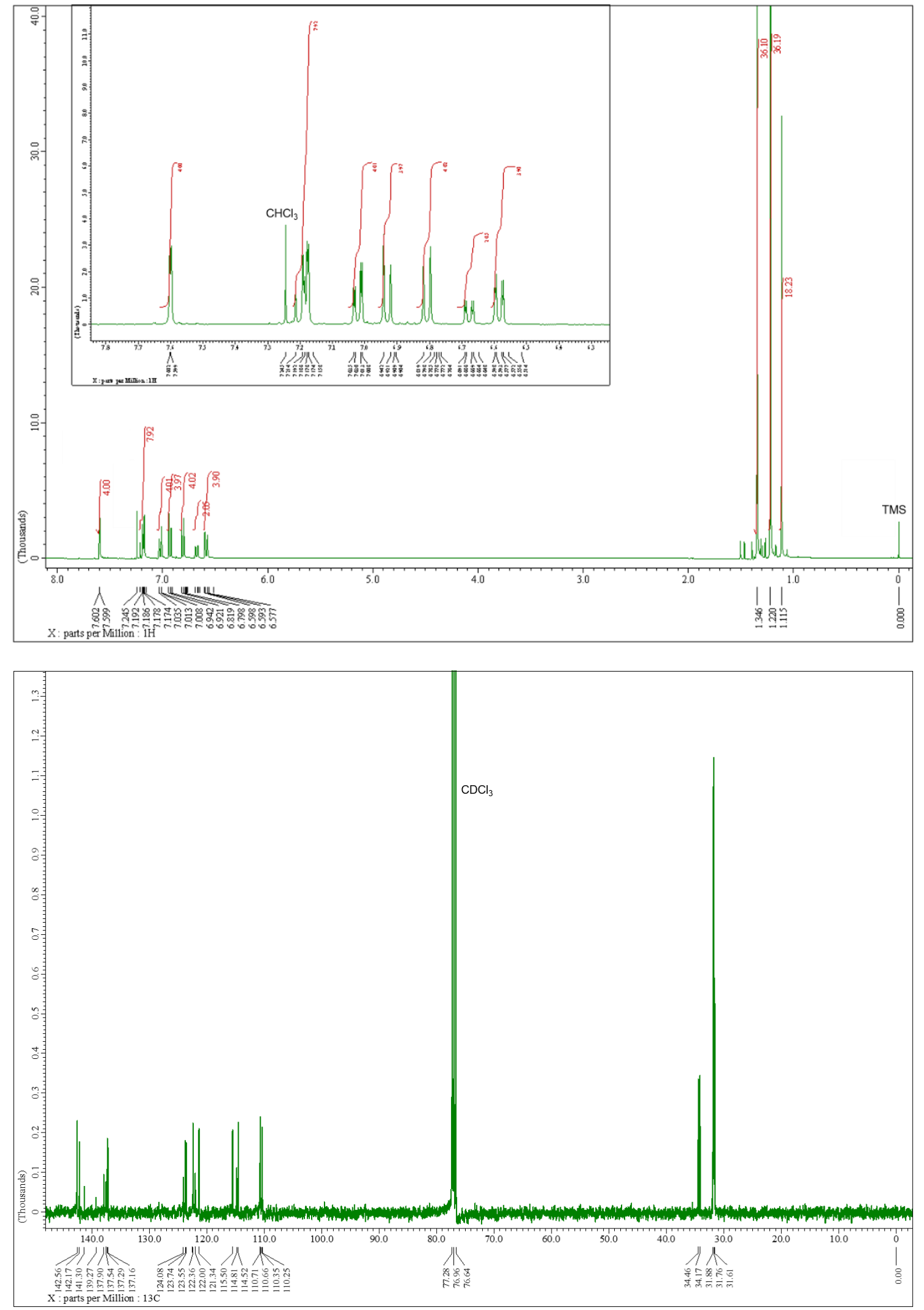

Figure S9. ${ }^{1} \mathrm{H}$ and ${ }^{13} \mathrm{C}\left\{{ }^{1} \mathrm{H}\right\}$ NMR spectra of 9 in $\mathrm{CDCl}_{3}$. 

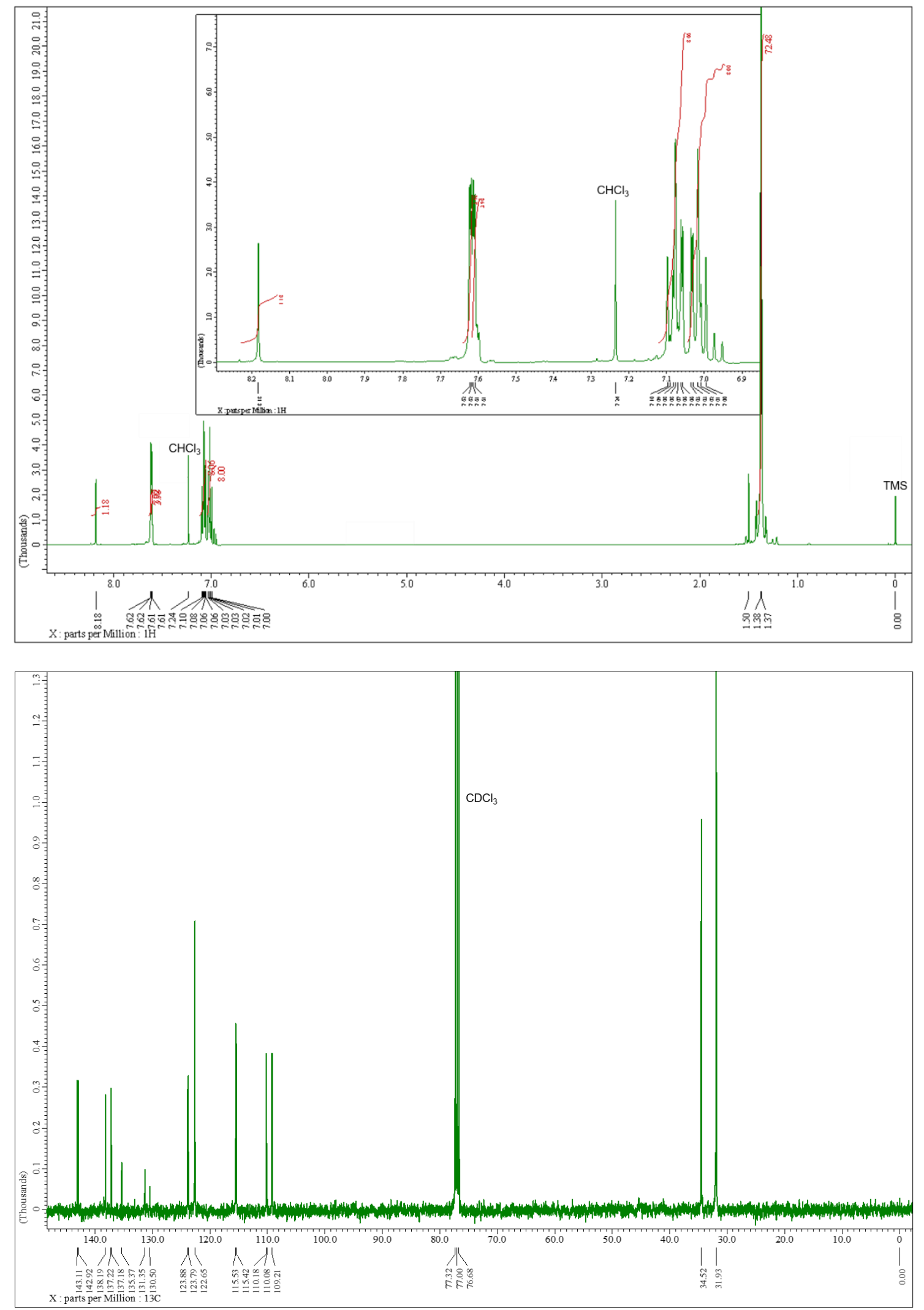

Figure S10. ${ }^{1} \mathrm{H}$ and ${ }^{13} \mathrm{C}\left\{{ }^{1} \mathrm{H}\right\}$ NMR spectra of $\mathbf{1 0}$ in $\mathrm{CDCl}_{3}$. 

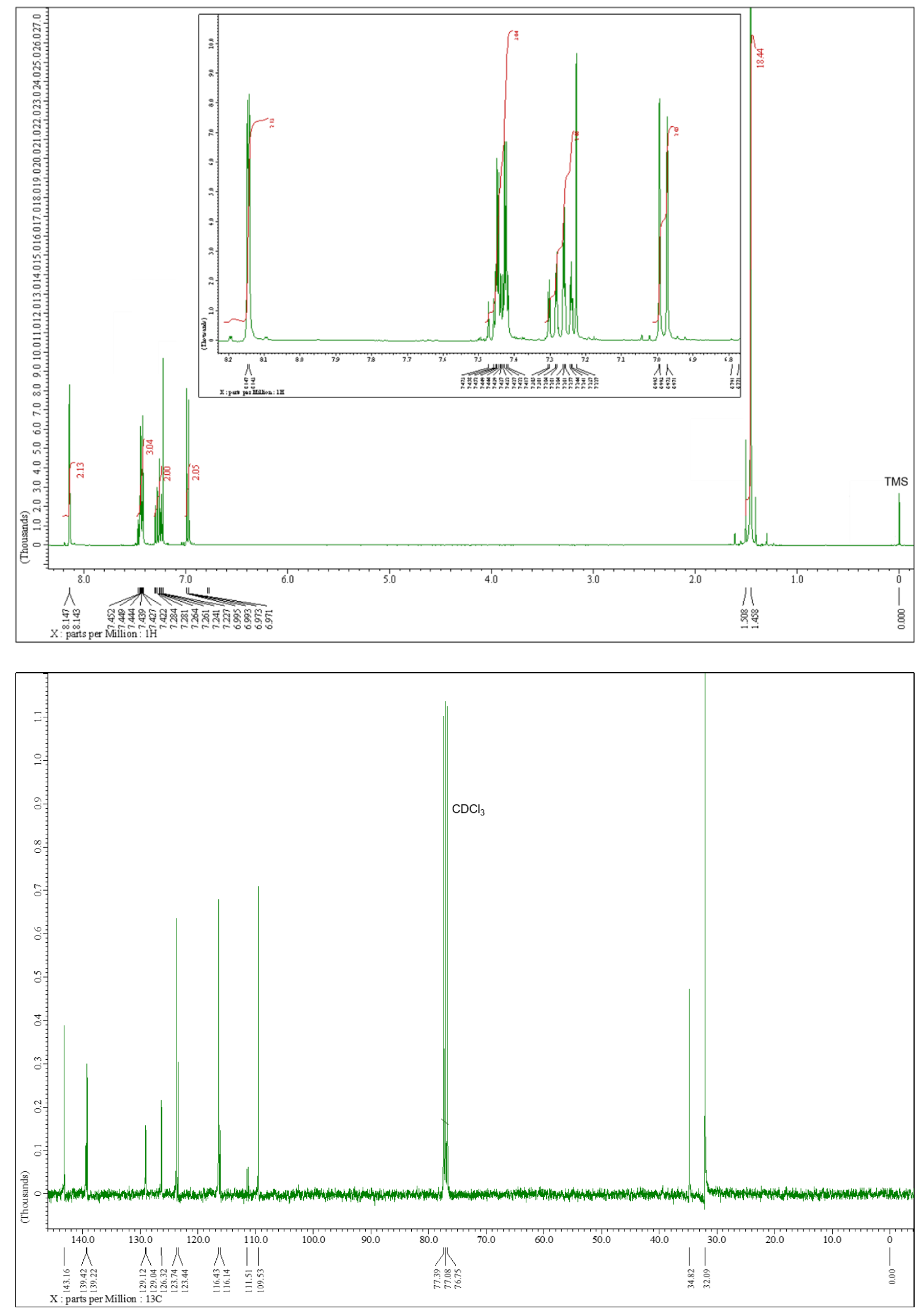

Figure S11. ${ }^{1} \mathrm{H}$ and ${ }^{13} \mathrm{C}\left\{{ }^{1} \mathrm{H}\right\}$ NMR spectra of $\mathbf{1 1}$ in $\mathrm{CDCl}_{3}$. 

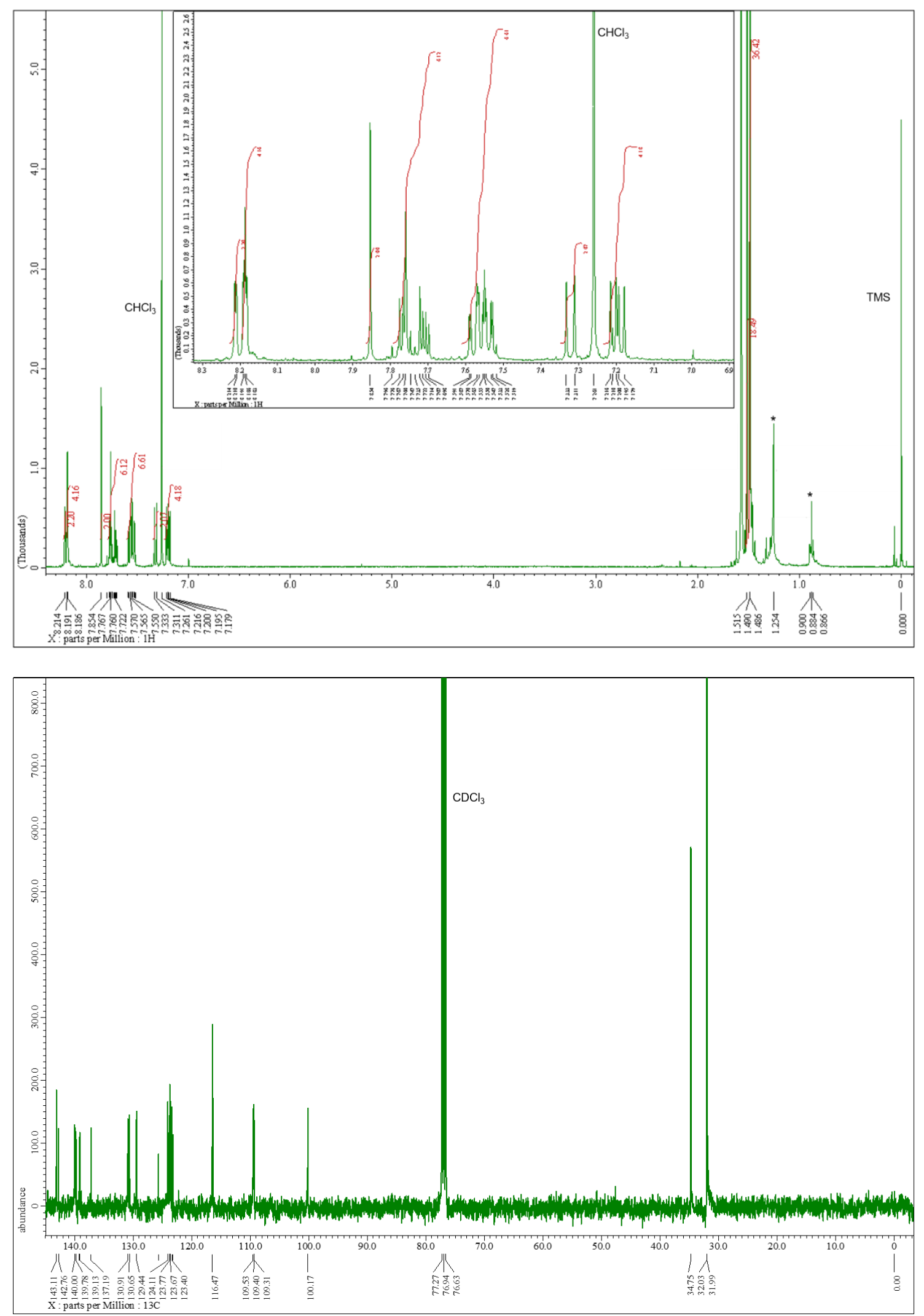

Figure S12. ${ }^{1} \mathrm{H}$ and ${ }^{13} \mathrm{C}\left\{{ }^{1} \mathrm{H}\right\}$ NMR spectra of $\mathbf{1 2}$ in $\mathrm{CDCl}_{3}$. ${ }^{*}$ Solvent impurities. 
Table S1. Crystallography data for $\mathbf{1}-\mathbf{5}$.

\begin{tabular}{|c|c|c|c|c|c|}
\hline $\begin{array}{l}\text { Compound } \\
\text { (CCDC no.) }\end{array}$ & $\begin{array}{c}1 \\
(2032784)\end{array}$ & $\begin{array}{c}2 \\
(2032785)\end{array}$ & $\begin{array}{c}\mathbf{3} \\
(2032786)\end{array}$ & $\begin{array}{c}4 \\
(2032787)\end{array}$ & $\begin{array}{c}\mathbf{5} \\
(2032788)\end{array}$ \\
\hline Empirical formula & $\mathrm{C}_{66} \mathrm{H}_{69} \mathrm{~B}_{2} \mathrm{~N}_{3}$ & $\mathrm{C}_{46} \mathrm{H}_{49} \mathrm{BN}_{2}$ & $\mathrm{C}_{106} \mathrm{H}_{118} \mathrm{BN}_{5}$ & $\mathrm{C}_{86} \mathrm{H}_{95} \mathrm{BN}_{4}$ & $\mathrm{C}_{78} \mathrm{H}_{76} \mathrm{~B}_{2} \mathrm{~N}_{4}$ \\
\hline Formula weight & 1094.68 & 640.68 & 1472.86 & 1314.83 & 1329.78 \\
\hline Temperature (K) & 100 & 100 & 100 & 100 & 100 \\
\hline Wavelength $(\AA)$ & 0.71073 & 0.71073 & 0.71073 & 0.71073 & 0.71073 \\
\hline Crystal system & monoclinic & monoclinic & triclinic & triclinic & triclinic \\
\hline Space group & $\mathrm{P}-1$ & $\mathrm{P}-1$ & $\mathrm{P}-1$ & $\mathrm{P}-1$ & P-1 \\
\hline$a(\AA)$ & $10.4145(3)$ & $27.2250(18)$ & $11.5713(3)$ & $10.2382(5)$ & $11.0522(4)$ \\
\hline$b(\AA)$ & $17.9165(6)$ & $5.6555(3)$ & $16.6467(4)$ & $16.6522(6)$ & $12.5935(5)$ \\
\hline$c(\AA)$ & $32.6339(9)$ & $22.6758(13)$ & $30.6126(7)$ & $24.0090(9)$ & $12.6285(5)$ \\
\hline$\alpha\left(^{\circ}\right)$ & 90 & 90 & $93.081(2)$ & $76.814(3)$ & $95.829(3)$ \\
\hline$\beta\left(^{\circ}\right)$ & $95.738(3)$ & $96.747(6)$ & $100.222(2)$ & $82.531(3)$ & $98.135(3)$ \\
\hline$\gamma\left({ }^{\circ}\right)$ & 90 & 90 & $107.876(2)$ & $79.275(3)$ & 105.999(3) \\
\hline Volume $\left(\AA^{3}\right)$ & $6058.7(3)$ & $3467.2(4)$ & $5486.0(2)$ & $3899.3(3)$ & $1654.28(11)$ \\
\hline$Z$ & 4 & 4 & 2 & 2 & 1 \\
\hline$D_{\text {calcd }}\left(\mathrm{g} \mathrm{cm}^{-3}\right)$ & 1.200 & 1.227 & 0.892 & 1.120 & 1.335 \\
\hline Absorption coeff. $\left(\mathrm{mm}^{-1}\right)$ & 0.132 & 0.070 & 0.051 & 0.163 & 0.310 \\
\hline$F(000)$ & 2332 & 1376 & 1588 & 1404 & 698 \\
\hline Reflections collected & 71828 & 10016 & 105565 & 54377 & 27811 \\
\hline Independent reflections & 13909 & 10016 & 25210 & 17779 & 7580 \\
\hline Completeness (\%) & $\begin{array}{c}0.999 \\
\left(\theta=25.242^{\circ}\right)\end{array}$ & $\begin{array}{c}0.990 \\
\left(\theta=25.242^{\circ}\right)\end{array}$ & $\begin{array}{c}0.999 \\
\left(\theta=25.242^{\circ}\right)\end{array}$ & $\begin{array}{c}0.994 \\
\left(\theta=25.242^{\circ}\right)\end{array}$ & $\begin{array}{c}0.999 \\
\left(\theta=25.242^{\circ}\right)\end{array}$ \\
\hline Data/ restrains / parameters & $13909 / 0 / 968$ & $10016 / 0 / 455$ & $25210 / 0 / 1039$ & $17779 / 6 / 951$ & $7580 / 0 / 424$ \\
\hline Goodness-of-fit on $F^{2}$ & 1.104 & 0.972 & 1.043 & 1.049 & 1.082 \\
\hline Final $R$ indices & $R_{1}=0.0899$ & $R_{1}=0.0538$ & $R_{1}=0.1328$ & $R_{1}=0.0619$ & $R_{1}=0.0474$ \\
\hline$[I>2 \sigma(I)]$ & $\mathrm{w} R_{2}=0.2332$ & $\mathrm{w} R_{2}=0.1177$ & $\mathrm{w} R_{2}=0.3401$ & $\mathrm{w} R_{2}=0.1562$ & $\mathrm{w} R_{2}=0.1232$ \\
\hline \multirow[t]{2}{*}{$R$ indices (all data) } & $R_{1}=0.1129$ & $R_{1}=0.0775$ & $R_{1}=0.1208$ & $R_{1}=0.0896$ & $R_{1}=0.0608$ \\
\hline & $\mathrm{w} R_{2}=0.2470$ & $\mathrm{w} R_{2}=0.1248$ & $\mathrm{w} R 2=0.3475$ & $\mathrm{w} R R_{2}=0.1689$ & $\mathrm{w} R 2=0.1310$ \\
\hline
\end{tabular}




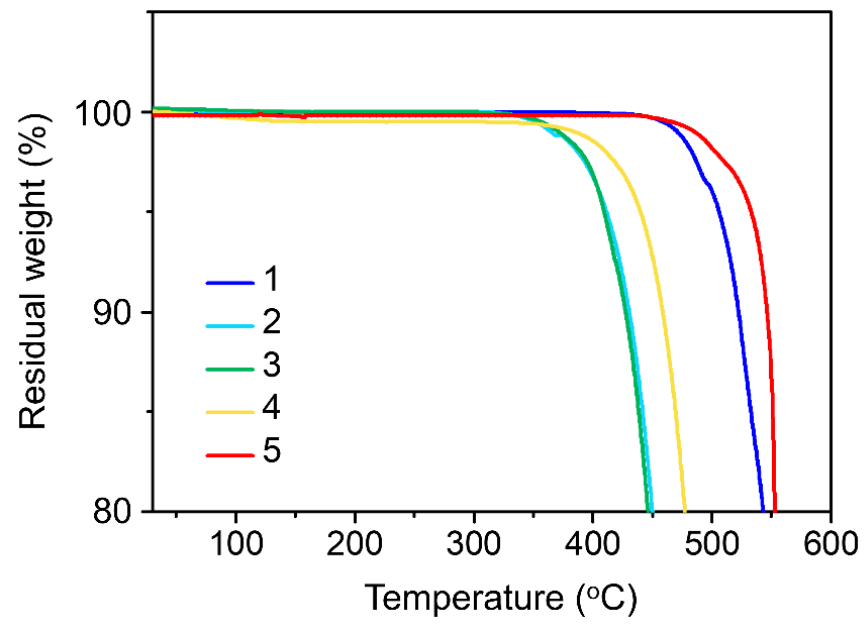

Figure S13. TGA thermograms of $1-5$ at a heating rate of $10{ }^{\circ} \mathrm{C} \min ^{-1}$ under $\mathrm{N}_{2}$.

Table S2. UV/vis absorption data of $\mathbf{1 - 5}$ in toluene solutions (cf. Table $3 \mathrm{a}$ )

\begin{tabular}{cccccc}
\hline Compd & $\mathbf{1}$ & $\mathbf{2}$ & $\mathbf{3}$ & $\mathbf{4}$ & $\mathbf{5}$ \\
\hline $\begin{array}{c}\lambda_{\mathrm{abs}} \\
(\mathrm{nm})\end{array}$ & 453 & 468 & 487 & 505 & 597 \\
$\begin{array}{c}\varepsilon \\
\left(10^{4} \mathrm{~cm}^{-1} \mathrm{M}^{-1}\right)\end{array}$ & 11.5 & 6.2 & 4.2 & 3.2 & 11.4 \\
\hline
\end{tabular}

a)
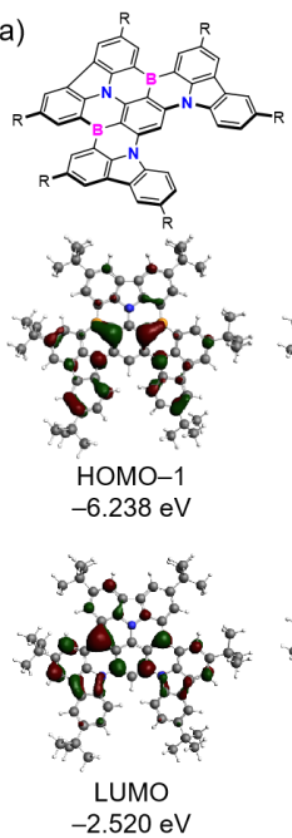

b)

$\mathrm{S}_{1}=2.8360 \mathrm{eV}\left(@ \mathrm{~S}_{0}\right)$ $\mathrm{T}_{1}=2.5843 \mathrm{eV}\left(@ \mathrm{~S}_{0}\right)$ $\Delta E_{\mathrm{ST}}=0.2517 \mathrm{eV}$ $f=0.1177\left(S_{0} \rightarrow S_{1}\right)$
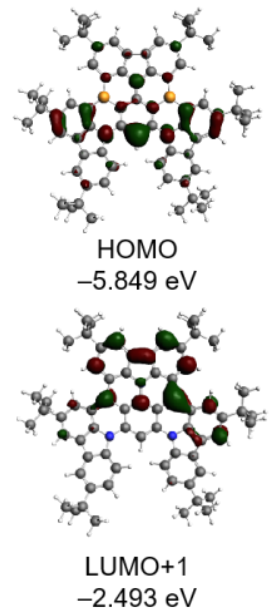

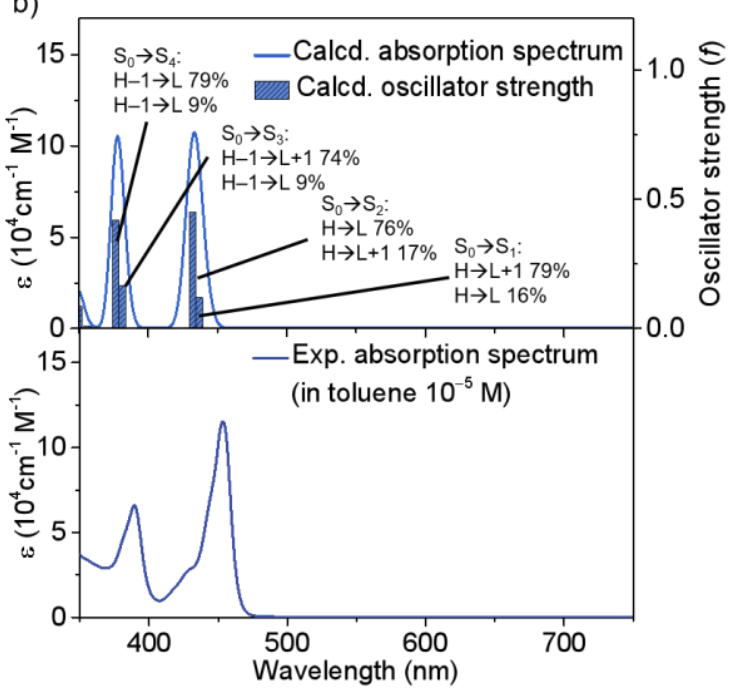

Figure S14. (a) Frontier molecular orbitals of 1 at an optimized $\mathrm{S}_{0}$ geometry and (b) theoretical absorption spectrum of $\mathbf{1}$ simulated with DFT at the PBE/DZP (top) and corresponding experimental absorption spectrum measured in toluene solution (bottom). 
a)

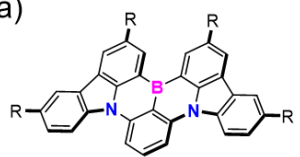

$=2.7265 \mathrm{eV}\left(@ \mathrm{~S}_{0}\right)$

$\mathrm{T}_{1}=2.4477 \mathrm{eV}\left(@ \mathrm{~S}_{0}\right)$

$\Delta E_{\mathrm{ST}}=0.2788 \mathrm{eV}$

$f=0.48653\left(S_{0} \rightarrow S_{1}\right)$
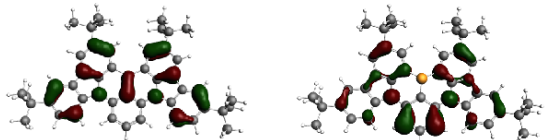

HOMO-1

$-6.457 \mathrm{eV}$

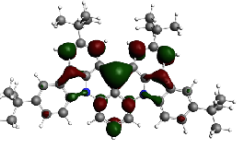

LUMO

$-2.479 \mathrm{eV}$
HOMO

$-5.767 \mathrm{eV}$

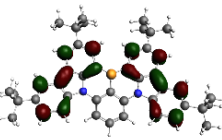

LUMO+1

$-1.488 \mathrm{eV}$

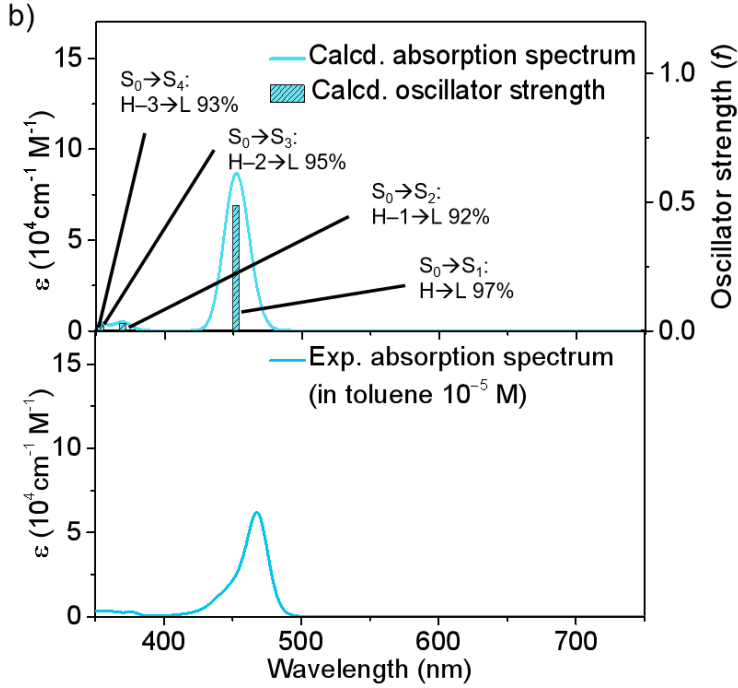

Figure S15. Frontier molecular orbitals of 2 at an optimized $\mathrm{S}_{0}$ geometry and (b) theoretical absorption spectrum of $\mathbf{2}$ simulated with DFT at the PBE/DZP (top) and corresponding experimental absorption spectrum measured in toluene solution (bottom).

a)

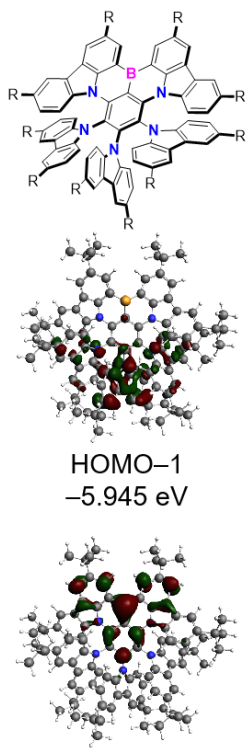

LUMO
$\mathrm{S}_{1}=2.4983 \mathrm{eV}\left(@ \mathrm{~S}_{0}\right)$

$\mathrm{T}_{1}=2.2849 \mathrm{eV}\left(@ \mathrm{~S}_{0}\right)$

$\Delta E_{\mathrm{ST}}=0.2134 \mathrm{eV}$

$f=0.19546\left(\mathrm{~S}_{0} \rightarrow \mathrm{S}_{1}\right)$

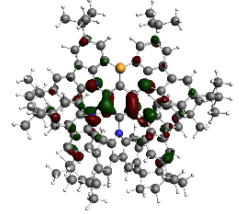

HOMO

$-5.814 \mathrm{eV}$

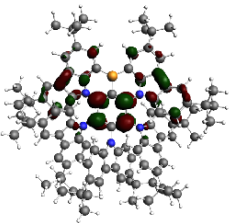

LUMO+1

$-1.882 \mathrm{eV}$ b)

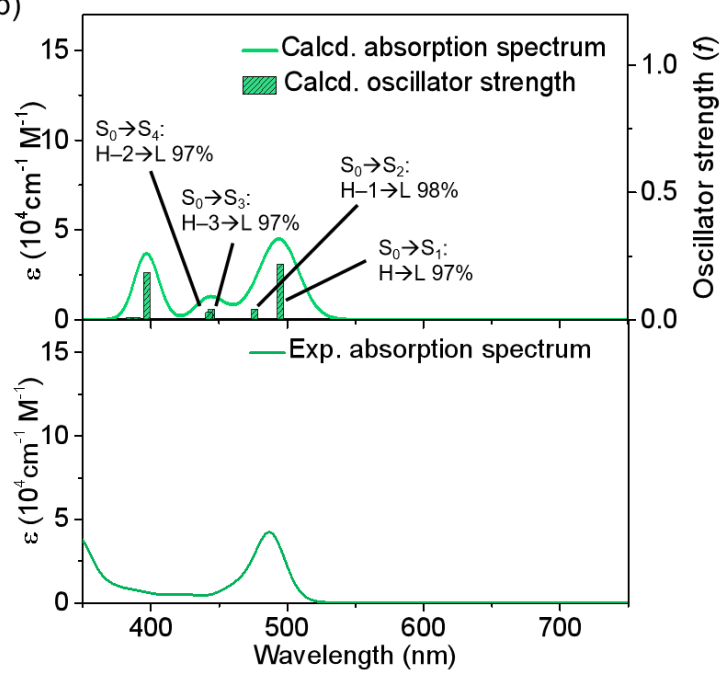

Figure S16. Frontier molecular orbitals of 3 at an optimized $\mathrm{S}_{0}$ geometry and (b) theoretical absorption spectrum of $\mathbf{3}$ simulated with DFT at the PBE/DZP (top) and corresponding experimental absorption spectrum measured in toluene solution (bottom). 

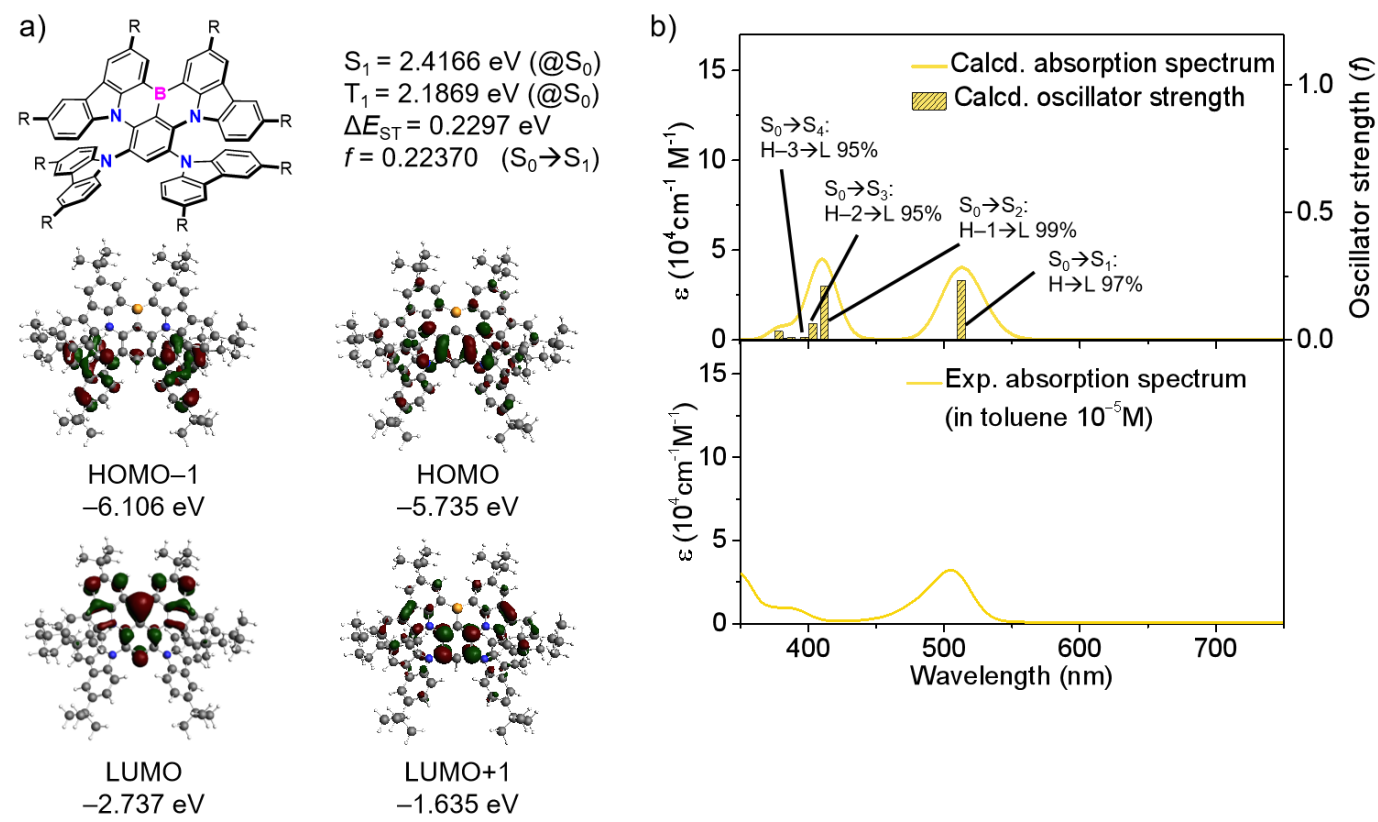

Figure S17. Frontier molecular orbitals of 4 at an optimized $\mathrm{S}_{0}$ geometry and (b) theoretical absorption spectrum of $\mathbf{4}$ simulated with DFT at the PBE/DZP (top) and corresponding experimental absorption spectrum measured in toluene solution (bottom).

a)
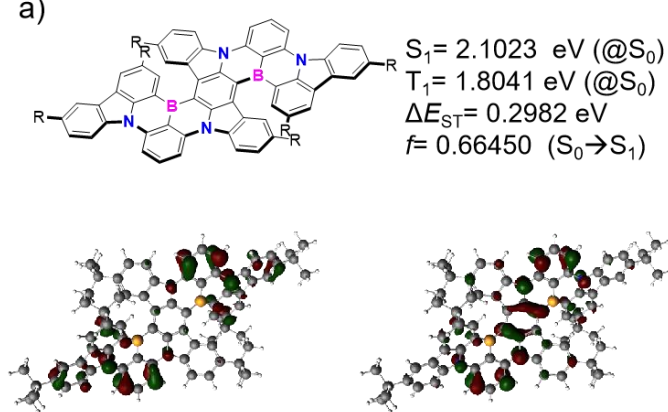

HOMO-1 $-6.118 \mathrm{eV}$

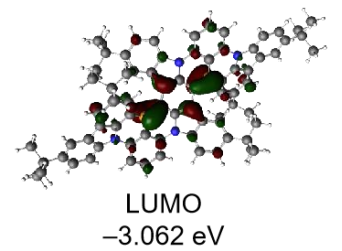

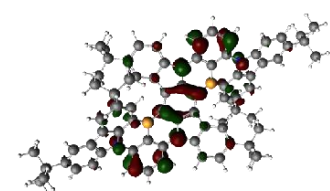

HOMO $-5.686 \mathrm{eV}$

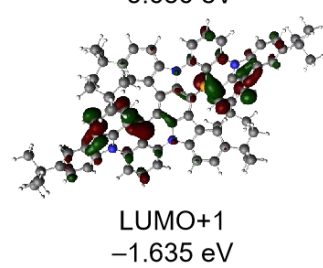

b)

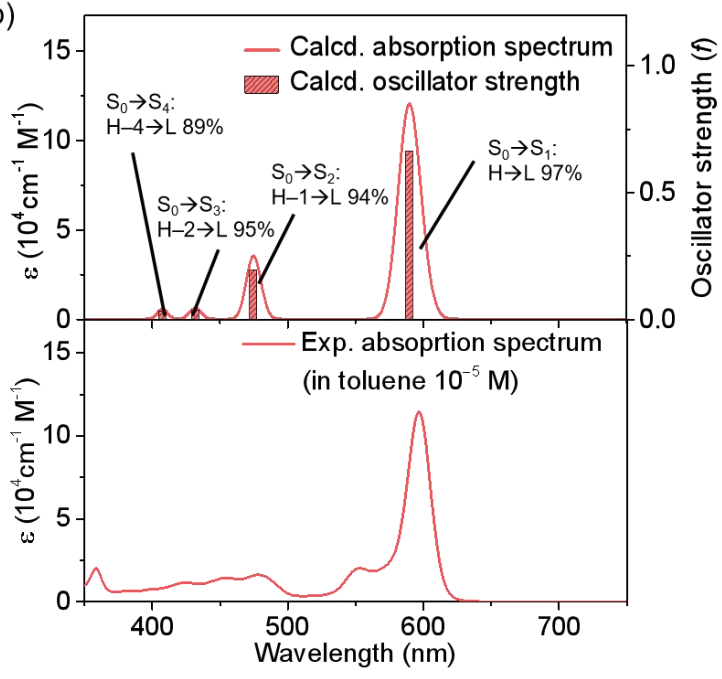

Figure S18. Frontier molecular orbitals of $\mathbf{5}$ at an optimized $\mathrm{S}_{0}$ geometry and (b) theoretical absorption spectrum of $\mathbf{5}$ simulated with DFT at the PBE/DZP (top) and corresponding experimental absorption spectrum measured in toluene solution (bottom). 

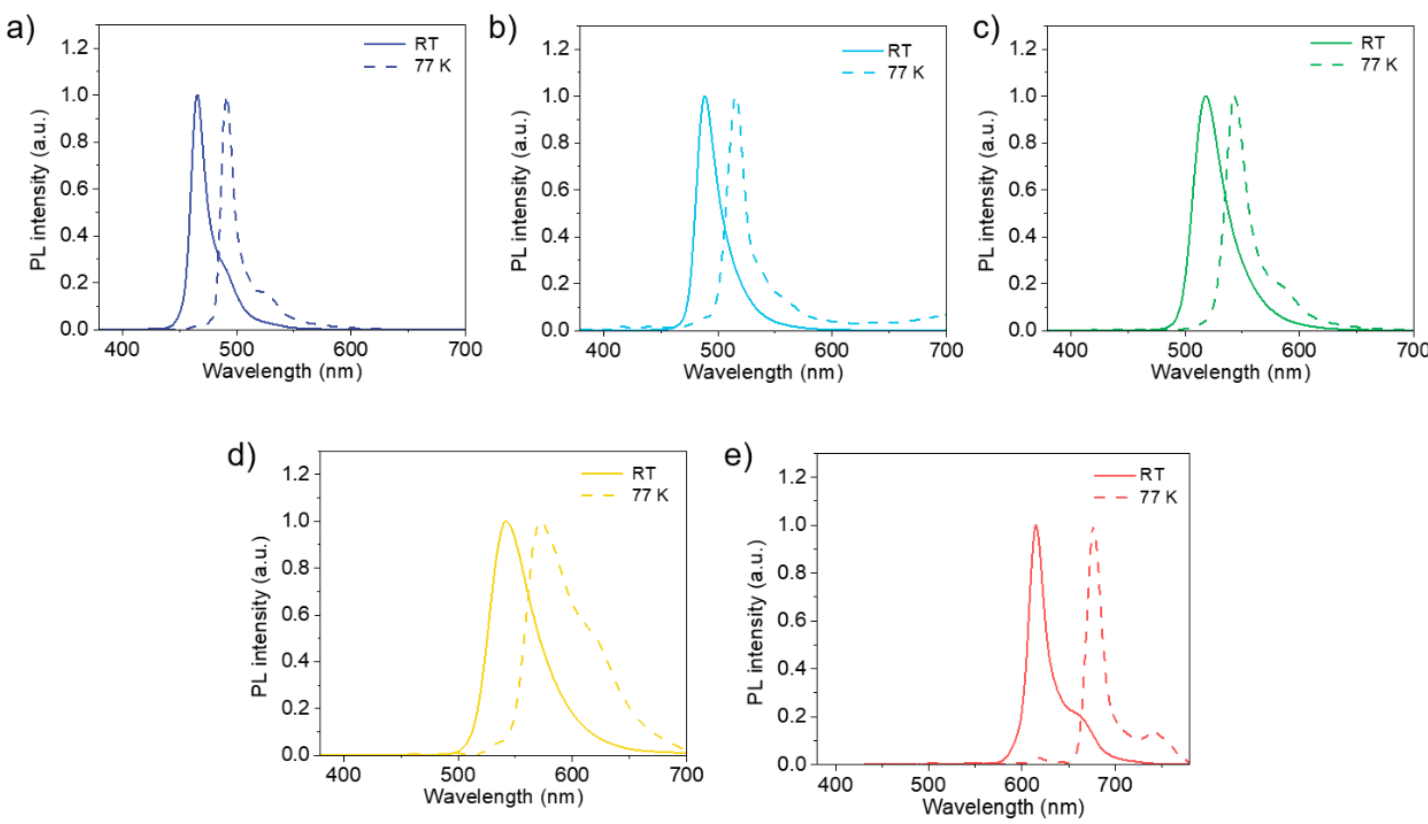

Figure S19. Fluorescence (300 K) and phosphorescence (77 K) spectra of (a) 1, (b) 2, (c) 3, (d) 4, and (e) 5 in toluene solutions $\left(10^{-5} \mathrm{M}\right)$.
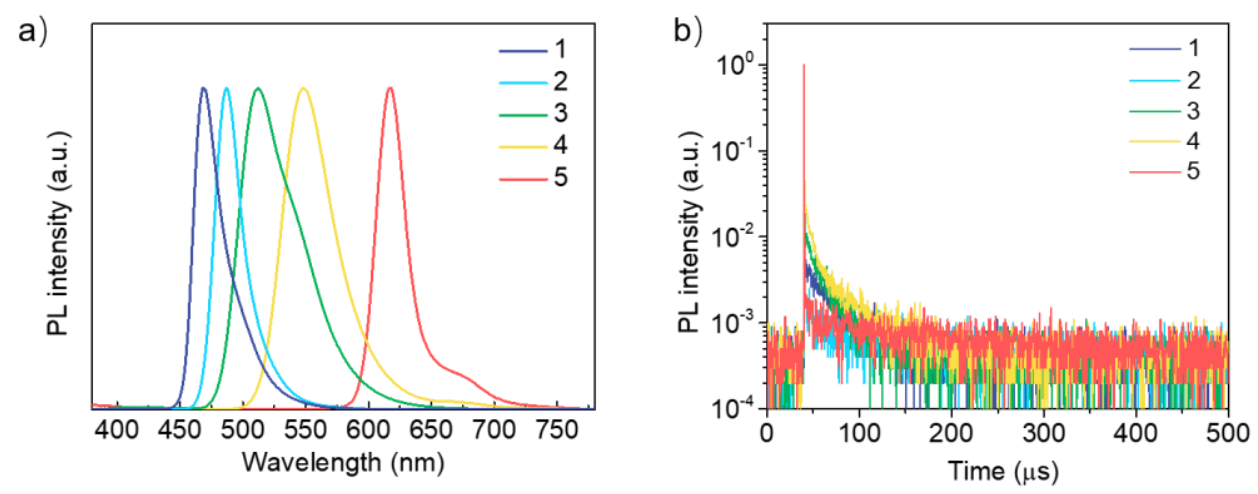

Figure S20. (a) PL spectra and (b) transient PL decay curves of 1-5 in $2 \mathrm{wt} \%$-doped films with mCBP host.

Table S3. Photophysical data of $\mathbf{1}-\mathbf{5}$ in $2 \mathrm{wt} \%$-doped films with mCBP host

\begin{tabular}{ccccccccc}
\hline Compd & $\begin{array}{c}\lambda_{\mathrm{PL}} \\
(\mathrm{nm})\end{array}$ & $\begin{array}{c}\mathrm{FWHM} \\
(\mathrm{nm} / \mathrm{eV})\end{array}$ & $\begin{array}{c}\mathrm{CIE} \\
(\mathrm{x}, \mathrm{y})\end{array}$ & $\begin{array}{c}\Phi_{\mathrm{PL}} \\
(\%)\end{array}$ & $\begin{array}{c}\tau_{\mathrm{p}} \\
(\mathrm{ns})\end{array}$ & $\begin{array}{c}\tau_{\mathrm{d}} \\
(\mu \mathrm{s})\end{array}$ & $\begin{array}{c}k_{\mathrm{r}} \\
\left(10^{7} \mathrm{~s}^{-1}\right)\end{array}$ & $\begin{array}{c}k_{\mathrm{RISC}} \\
\left(10^{4} \mathrm{~s}^{-1}\right)\end{array}$ \\
\hline $\mathbf{1}$ & 471 & $26 / 0.14$ & $(0.12,0.20)$ & 91 & 4.2 & 35 & 19 & 2.2 \\
$\mathbf{2}$ & 490 & $25 / 0.13$ & $(0.09,0.40)$ & 98 & 3.7 & 43 & 20 & 2.0 \\
$\mathbf{3}$ & 519 & $50 / 0.25$ & $(0.23,0.65)$ & 99 & 4.7 & 13 & 8.0 & 19 \\
$\mathbf{4}$ & 549 & $48 / 0.19$ & $(0.39,0.59)$ & 90 & 5.7 & 11 & 7.3 & 11 \\
$\mathbf{5}$ & 619 & $27 / 0.08$ & $(0.68,0.32)$ & 79 & 5.1 & 53 & 13 & 1.2 \\
\hline
\end{tabular}


a)

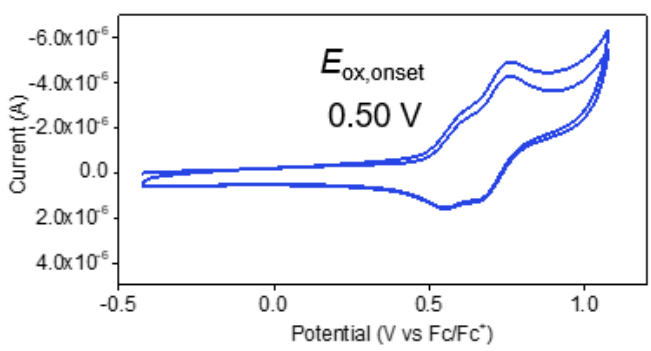

b)

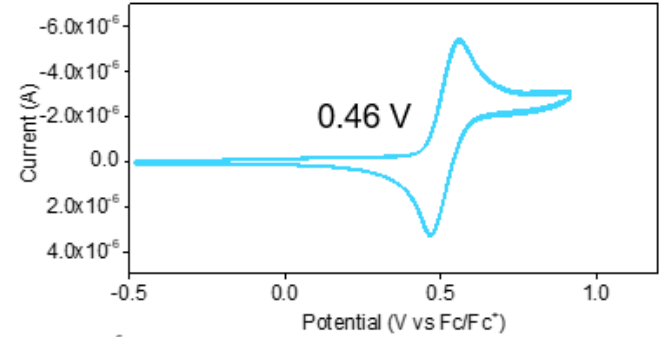

c)

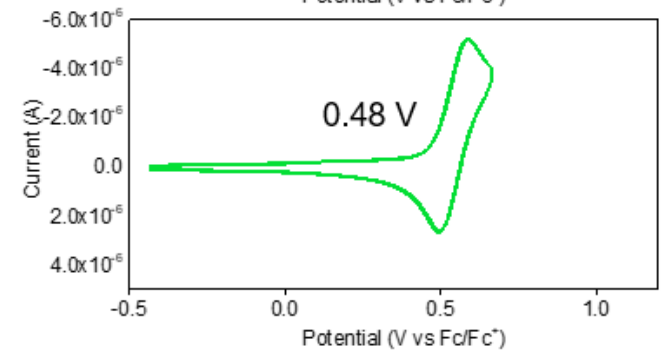

d)

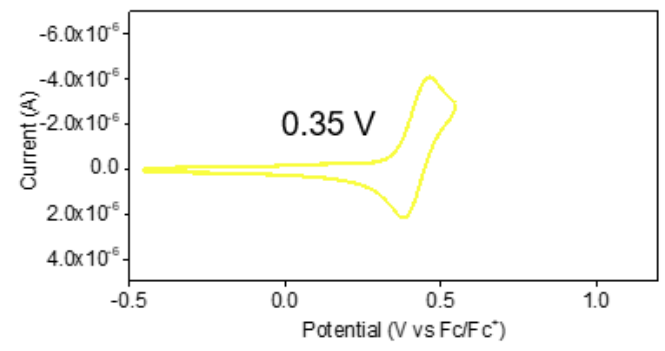

e)

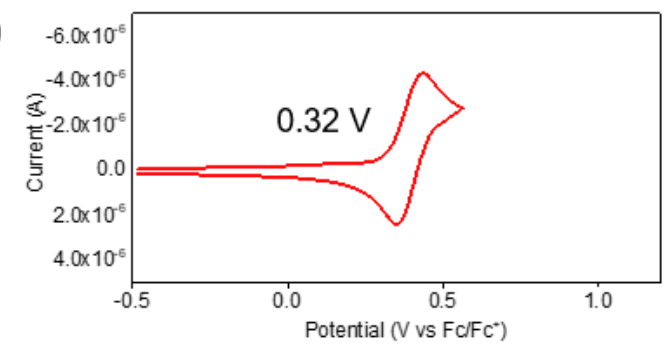

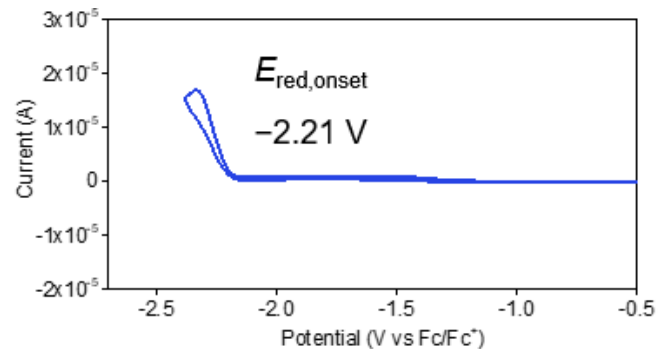
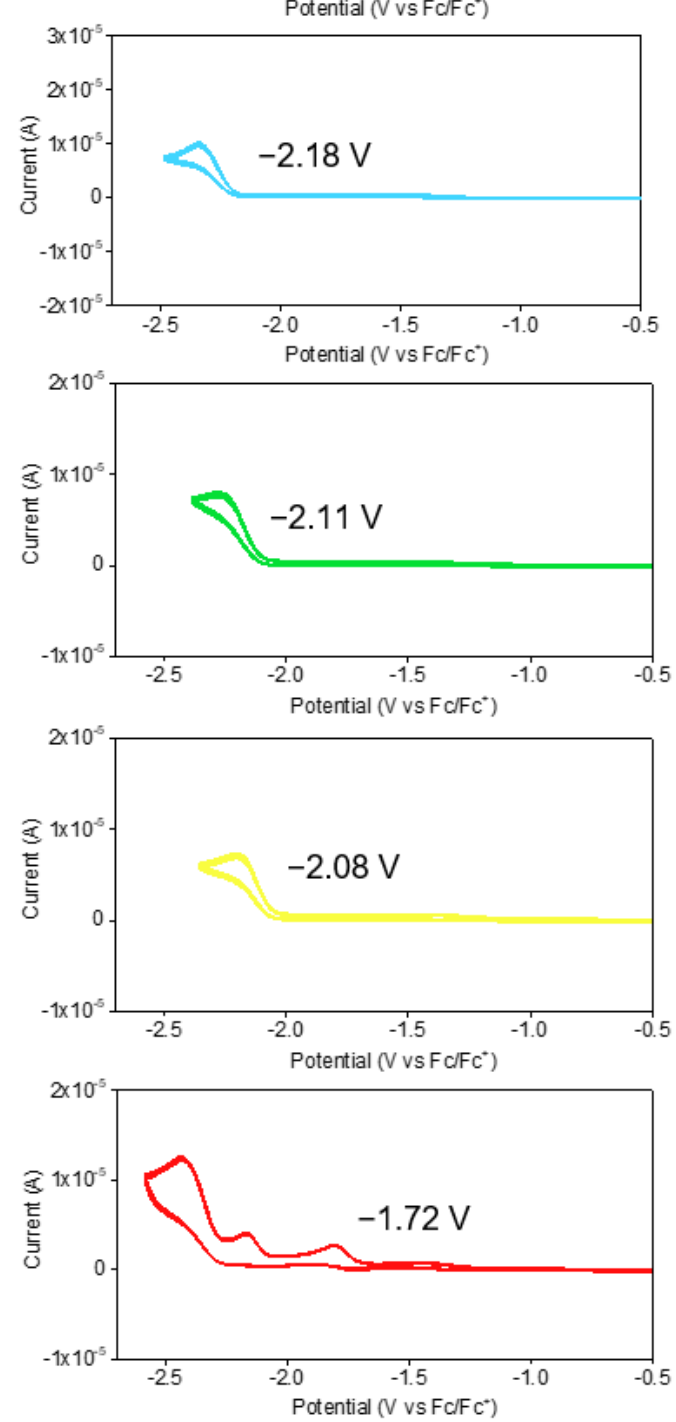

Figure S21. Cyclic voltammograms of (a) 1, (b) 2, (c) 3, (d) 4, and (e) 5 in $\mathrm{Bu}_{4} \mathrm{NClO}_{4} / \mathrm{CH}_{2} \mathrm{Cl}_{2}$ $(0.1 \mathrm{M})$ electrolyte measured at a scanning rate of $100 \mathrm{mV} \mathrm{s}^{-1}$. 


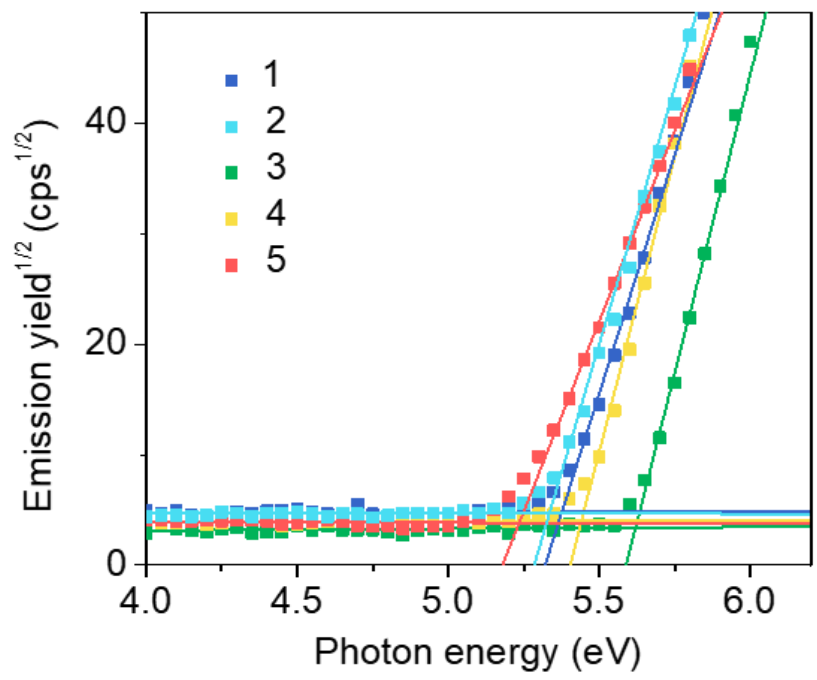

Figure S22. Photoelectron yield spectra of $\mathbf{1}-\mathbf{5}$ in pure neat films.

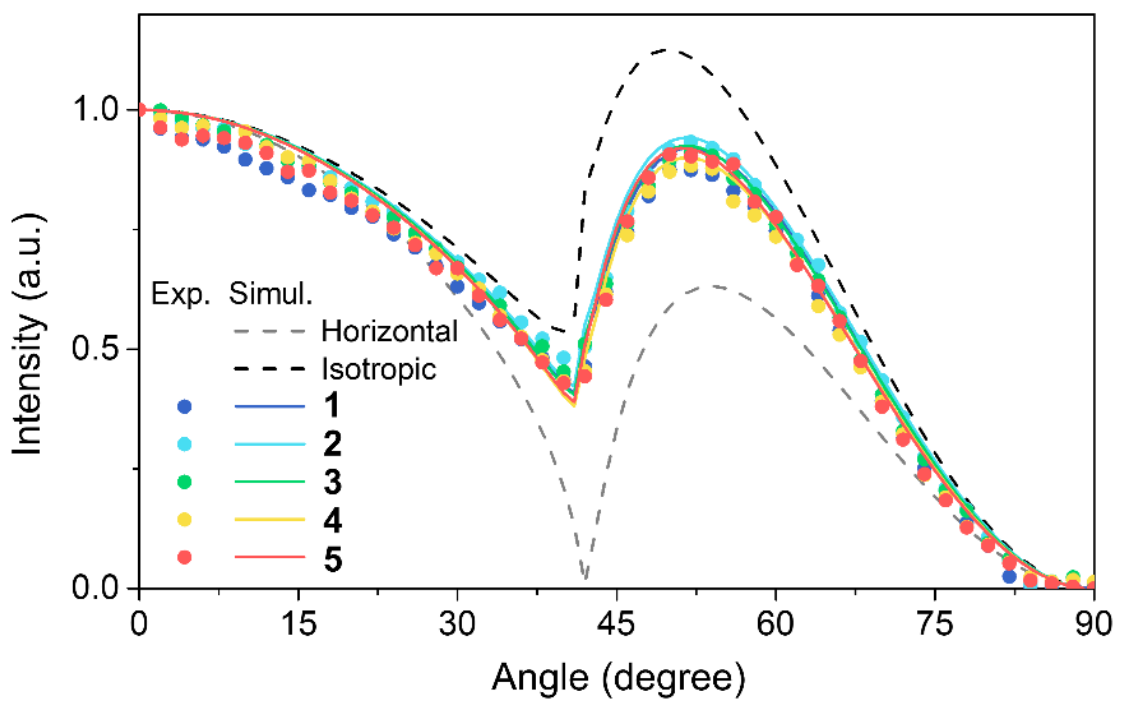

Figure S23. Angle dependent p-polarized PL characteristics of 1-5 in the $2 \mathrm{wt} \%$ doped films. Horizontal transition dipole ratios $(\Theta)$ were calculated to be $0.74,0.73,0.74,0.75$, and 0.74 for $\mathbf{1}-\mathbf{5}$, respectively. 


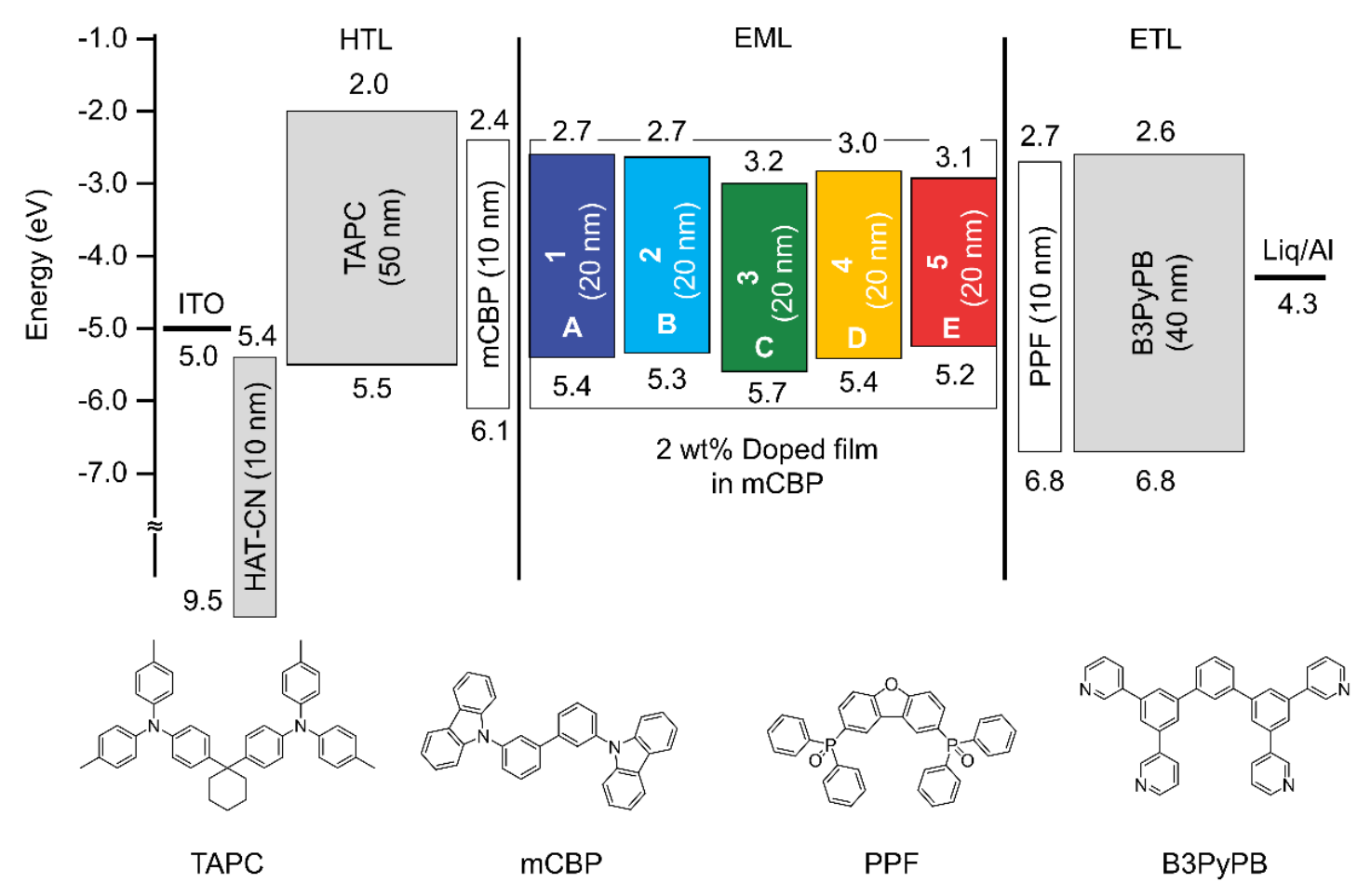

Figure S24. Energy level diagram of OLEDs based on 1-5 (devices A-E) and molecular structures of used materials.

(a)

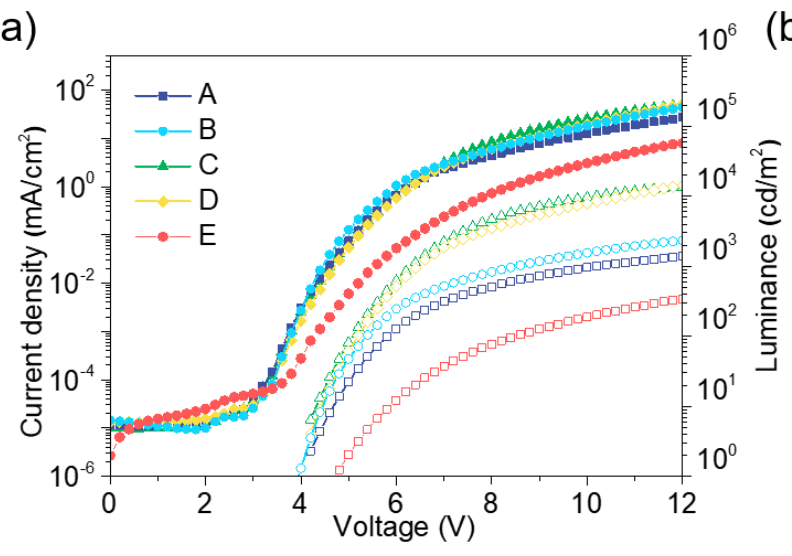

(b)

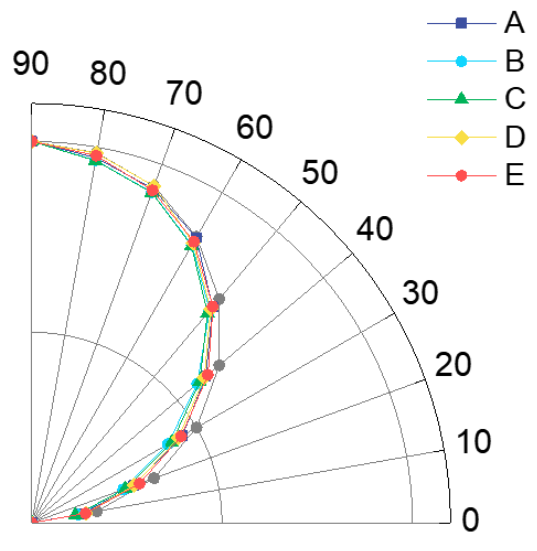

Figure S25. (a) Current density-voltage-luminance $(J-V-L)$ characteristics and (b) angular dependence of the EL intensities with Lambertian distributions of devices A-E. Lambertian factors were calculated to be $0.933,0.893,0.890,0.929$, and 0.943 for devices A-E, respectively. 


\section{References}

(1) Zhu, C.; Ji, X.; You, D.; Chen, T. L.; Mu, A. U.; Barker, K. P.; Klivansky, L. M.; Liu, Y.; Fang, L. J. Am. Chem. Soc. 2018, 140, 18173-18182.

(2) Vecchi, P. A.; Padmaperuma, A. B.; Qiao, H.; Sapochak, L. S.; Burrows, P. E. A. Org. Lett. 2006, 8, 4211-4214.

(3) ADF2018, SCM, Theoretical Chemistry, Vrije Universiteit, Amsterdam, The Netherlands, http://www.scm.com/

(4) (a) Lee, J.; Aizawa, N.; Numata, M.; Adachi, C.; Yasuda, T. Adv. Mater. 2017, 29, 1604856.

(b) Park, I. S.; Matsuo, K.; Aizawa, N.; Yasuda, T. Adv. Funct. Mater. 2018, 28, 1802031. (c)

Matsuo, K.; Yasuda, T. Chem. Sci. 2019, 10, 10687-10697.

(5) Kim, K. H.; Kim, J. J. Adv. Mater. 2018, 30, 1-19. 


\section{Computational geometry data}

Geometry data for $\mathbf{1}$ (S $\mathrm{S}_{0}$ optimization: unit $\AA$ )

\begin{tabular}{|c|c|c|c|}
\hline $\mathrm{C}$ & -1.327600000000 & 0.444000000000 & 2.596700000000 \\
\hline $\mathrm{C}$ & -0.024300000000 & 0.146600000000 & 2.141300000000 \\
\hline $\mathrm{C}$ & 0.236200000000 & 0.496800000000 & 0.790200000000 \\
\hline $\mathrm{C}$ & -0.774600000000 & 0.985300000000 & -0.037800000000 \\
\hline $\mathrm{C}$ & -2.062600000000 & 1.202800000000 & 0.453500000000 \\
\hline $\mathrm{C}$ & -2.373200000000 & 0.993800000000 & 1.822500000000 \\
\hline $\mathrm{C}$ & 2.129700000000 & 0.659500000000 & -0.919400000000 \\
\hline $\mathrm{C}$ & 2.471200000000 & -0.431300000000 & 1.038900000000 \\
\hline $\mathrm{C}$ & 1.715600000000 & 1.491000000000 & -1.953900000000 \\
\hline $\mathrm{C}$ & 3.409700000000 & 0.068400000000 & -0.982800000000 \\
\hline $\mathrm{C}$ & 2.327400000000 & -0.911300000000 & 2.342500000000 \\
\hline $\mathrm{C}$ & 3.622900000000 & -0.628300000000 & 0.271100000000 \\
\hline $\mathrm{C}$ & 2.553800000000 & 1.660200000000 & -3.053100000000 \\
\hline $\mathrm{C}$ & 4.223900000000 & 0.252100000000 & -2.090200000000 \\
\hline $\mathrm{C}$ & 3.414900000000 & -1.656500000000 & 2.819800000000 \\
\hline $\mathrm{C}$ & 4.674400000000 & -1.366800000000 & 0.795300000000 \\
\hline $\mathrm{C}$ & 3.804800000000 & 1.043600000000 & -3.160400000000 \\
\hline $\mathrm{H}$ & 2.205900000000 & 2.312200000000 & -3.844900000000 \\
\hline $\mathrm{H}$ & 5.201600000000 & -0.220400000000 & -2.107400000000 \\
\hline $\mathrm{C}$ & 4.580600000000 & -1.903000000000 & 2.083600000000 \\
\hline $\mathrm{H}$ & 3.343700000000 & -2.075700000000 & 3.816600000000 \\
\hline $\mathrm{H}$ & 5.563700000000 & -1.523400000000 & 0.195700000000 \\
\hline $\mathrm{C}$ & -4.285900000000 & 2.141400000000 & 0.152700000000 \\
\hline $\mathrm{C}$ & -3.129900000000 & 1.849700000000 & -1.777500000000 \\
\hline $\mathrm{C}$ & -4.695300000000 & 2.079600000000 & 1.483100000000 \\
\hline $\mathrm{C}$ & -5.064800000000 & 2.706000000000 & -0.866600000000 \\
\hline $\mathrm{C}$ & -2.283900000000 & 1.440200000000 & -2.801700000000 \\
\hline $\mathrm{C}$ & -4.328000000000 & 2.520200000000 & -2.102700000000 \\
\hline $\mathrm{C}$ & -5.942500000000 & 2.673700000000 & 1.751600000000 \\
\hline $\mathrm{C}$ & -6.287400000000 & 3.269000000000 & -0.549600000000 \\
\hline C & -2.616000000000 & 1.765400000000 & -4.114700000000 \\
\hline $\mathrm{C}$ & -4.634100000000 & 2.833100000000 & -3.418200000000 \\
\hline $\mathrm{C}$ & -6.744600000000 & 3.269800000000 & 0.777700000000 \\
\hline $\mathrm{H}$ & -6.287300000000 & 2.674400000000 & 2.776300000000 \\
\hline $\mathrm{H}$ & -6.891900000000 & 3.713300000000 & -1.335000000000 \\
\hline $\mathrm{C}$ & -3.772700000000 & 2.473600000000 & -4.455900000000 \\
\hline $\mathrm{H}$ & -1.938400000000 & 1.435200000000 & -4.892500000000 \\
\hline $\mathrm{H}$ & -5.563400000000 & 3.352700000000 & -3.632100000000 \\
\hline $\mathrm{N}$ & -3.085000000000 & 1.661800000000 & -0.374300000000 \\
\hline $\mathrm{N}$ & 1.532400000000 & 0.306500000000 & 0.314700000000 \\
\hline $\mathrm{C}$ & 4.718600000000 & 1.225100000000 & -4.378600000000 \\
\hline $\mathrm{C}$ & 4.090300000000 & 2.124200000000 & -5.449800000000 \\
\hline $\mathrm{C}$ & 6.046400000000 & 1.865000000000 & -3.935300000000 \\
\hline $\mathrm{C}$ & 5.002500000000 & -0.148100000000 & -5.013100000000 \\
\hline $\mathrm{H}$ & 3.148300000000 & 1.712100000000 & -5.825400000000 \\
\hline $\mathrm{H}$ & 3.897600000000 & 3.133700000000 & -5.073000000000 \\
\hline $\mathrm{H}$ & 4.773500000000 & 2.215000000000 & -6.300100000000 \\
\hline $\mathrm{H}$ & 6.570500000000 & 1.247000000000 & -3.200600000000 \\
\hline $\mathrm{H}$ & 6.712200000000 & 1.996400000000 & -4.795400000000 \\
\hline $\mathrm{H}$ & 5.875300000000 & 2.847300000000 & -3.483800000000 \\
\hline $\mathrm{H}$ & 5.661100000000 & -0.038300000000 & -5.881700000000 \\
\hline $\mathrm{H}$ & 5.490900000000 & -0.827800000000 & -4.308900000000 \\
\hline $\mathrm{H}$ & 4.075000000000 & -0.624000000000 & -5.347000000000 \\
\hline $\mathrm{C}$ & 5.705100000000 & -2.739900000000 & 2.709700000000 \\
\hline $\mathrm{C}$ & 5.174700000000 & -4.145600000000 & 3.044300000000 \\
\hline $\mathrm{C}$ & 6.905800000000 & -2.897200000000 & 1.769000000000 \\
\hline $\mathrm{C}$ & 6.198000000000 & -2.061400000000 & 4.000500000000 \\
\hline $\mathrm{H}$ & 4.340000000000 & -4.108000000000 & 3.750200000000 \\
\hline $\mathrm{H}$ & 4.824600000000 & -4.656300000000 & 2.141700000000 \\
\hline $\mathrm{H}$ & 5.965800000000 & -4.754700000000 & 3.495600000000 \\
\hline $\mathrm{H}$ & 7.352800000000 & -1.930900000000 & 1.513900000000 \\
\hline
\end{tabular}




\begin{tabular}{|c|c|c|c|}
\hline $\mathrm{H}$ & 7.678700000000 & -3.499300000000 & 2.256700000000 \\
\hline $\mathrm{H}$ & 6.630700000000 & -3.405000000000 & 0.839000000000 \\
\hline $\mathrm{H}$ & 7.001700000000 & -2.649300000000 & 4.457500000000 \\
\hline $\mathrm{H}$ & 6.585600000000 & -1.059100000000 & 3.791800000000 \\
\hline $\mathrm{H}$ & 5.398000000000 & -1.962100000000 & 4.739800000000 \\
\hline $\mathrm{C}$ & -4.127400000000 & 2.838000000000 & -5.902600000000 \\
\hline $\mathrm{C}$ & -4.251500000000 & 4.366900000000 & -6.028400000000 \\
\hline $\mathrm{C}$ & -3.065200000000 & 2.362300000000 & -6.900700000000 \\
\hline $\mathrm{C}$ & -5.468300000000 & 2.185500000000 & -6.283700000000 \\
\hline $\mathrm{H}$ & -5.026100000000 & 4.767300000000 & -5.368000000000 \\
\hline $\mathrm{H}$ & -3.307800000000 & 4.858400000000 & -5.771700000000 \\
\hline $\mathrm{H}$ & -4.512400000000 & 4.645400000000 & -7.055400000000 \\
\hline $\mathrm{H}$ & -2.953900000000 & 1.273400000000 & -6.889600000000 \\
\hline $\mathrm{H}$ & -3.357400000000 & 2.653100000000 & -7.914500000000 \\
\hline $\mathrm{H}$ & -2.087000000000 & 2.809600000000 & -6.697600000000 \\
\hline $\mathrm{H}$ & -5.737300000000 & 2.441100000000 & -7.314500000000 \\
\hline $\mathrm{H}$ & -5.408200000000 & 1.095300000000 & -6.207700000000 \\
\hline $\mathrm{H}$ & -6.282800000000 & 2.520600000000 & -5.635000000000 \\
\hline $\mathrm{C}$ & -8.099500000000 & 3.914100000000 & 1.101800000000 \\
\hline $\mathrm{C}$ & -8.071100000000 & 5.399800000000 & 0.700400000000 \\
\hline $\mathrm{C}$ & -9.211200000000 & 3.195800000000 & 0.315900000000 \\
\hline $\mathrm{C}$ & -8.445100000000 & 3.833200000000 & 2.593700000000 \\
\hline $\mathrm{H}$ & -7.293600000000 & 5.938400000000 & 1.251300000000 \\
\hline $\mathrm{H}$ & -7.873400000000 & 5.529700000000 & -0.367700000000 \\
\hline $\mathrm{H}$ & -9.034500000000 & 5.873300000000 & 0.919800000000 \\
\hline $\mathrm{H}$ & -9.259700000000 & 2.136600000000 & 0.587800000000 \\
\hline $\mathrm{H}$ & -10.185300000000 & 3.648300000000 & 0.532100000000 \\
\hline $\mathrm{H}$ & -9.050300000000 & 3.255400000000 & -0.764500000000 \\
\hline $\mathrm{H}$ & -9.415800000000 & 4.306800000000 & 2.771300000000 \\
\hline $\mathrm{H}$ & -8.515400000000 & 2.797400000000 & 2.940900000000 \\
\hline $\mathrm{H}$ & -7.706100000000 & 4.353300000000 & 3.211600000000 \\
\hline $\mathrm{C}$ & -2.807500000000 & 0.417300000000 & 4.543600000000 \\
\hline $\mathrm{C}$ & -0.722500000000 & -0.384400000000 & 4.816200000000 \\
\hline $\mathrm{C}$ & -3.921900000000 & 0.983100000000 & 3.941500000000 \\
\hline $\mathrm{C}$ & -2.717100000000 & 0.008300000000 & 5.887500000000 \\
\hline $\mathrm{C}$ & 0.578800000000 & -0.767800000000 & 4.525900000000 \\
\hline $\mathrm{C}$ & -1.357100000000 & -0.508200000000 & 6.066800000000 \\
\hline $\mathrm{C}$ & -5.035200000000 & 1.103500000000 & 4.799500000000 \\
\hline $\mathrm{C}$ & -3.837400000000 & 0.165900000000 & 6.680100000000 \\
\hline $\mathrm{C}$ & 1.279600000000 & -1.292800000000 & 5.632000000000 \\
\hline $\mathrm{C}$ & -0.621300000000 & -1.042800000000 & 7.106300000000 \\
\hline $\mathrm{C}$ & -5.019300000000 & 0.712800000000 & 6.139400000000 \\
\hline $\mathrm{H}$ & -5.951700000000 & 1.507200000000 & 4.392200000000 \\
\hline $\mathrm{H}$ & -3.809500000000 & -0.137500000000 & 7.722300000000 \\
\hline $\mathrm{C}$ & 0.716700000000 & -1.438100000000 & 6.900700000000 \\
\hline $\mathrm{H}$ & 2.310900000000 & -1.584600000000 & 5.490100000000 \\
\hline $\mathrm{H}$ & -1.073400000000 & -1.154500000000 & 8.087200000000 \\
\hline $\mathrm{N}$ & -1.603400000000 & 0.174400000000 & 3.910700000000 \\
\hline B & -3.729600000000 & 1.361700000000 & 2.447500000000 \\
\hline B & 1.009100000000 & -0.536900000000 & 3.052200000000 \\
\hline $\mathrm{C}$ & -6.247100000000 & 0.856800000000 & 7.051000000000 \\
\hline $\mathrm{C}$ & -5.901100000000 & 1.774500000000 & 8.237600000000 \\
\hline $\mathrm{C}$ & -7.453200000000 & 1.461500000000 & 6.322100000000 \\
\hline $\mathrm{C}$ & -6.659700000000 & -0.528100000000 & 7.581800000000 \\
\hline $\mathrm{H}$ & -5.073900000000 & 1.377800000000 & 8.833300000000 \\
\hline $\mathrm{H}$ & -5.612800000000 & 2.771300000000 & 7.889000000000 \\
\hline $\mathrm{H}$ & -6.766000000000 & 1.882500000000 & 8.901400000000 \\
\hline $\mathrm{H}$ & -7.776400000000 & 0.838100000000 & 5.482300000000 \\
\hline $\mathrm{H}$ & -8.296300000000 & 1.543400000000 & 7.015200000000 \\
\hline $\mathrm{H}$ & -7.239700000000 & 2.465800000000 & 5.942600000000 \\
\hline $\mathrm{H}$ & -7.532500000000 & -0.440700000000 & 8.238300000000 \\
\hline $\mathrm{H}$ & -6.919400000000 & -1.200300000000 & 6.757900000000 \\
\hline $\mathrm{H}$ & -5.858300000000 & -1.000400000000 & 8.157300000000 \\
\hline $\mathrm{C}$ & 1.504100000000 & -2.014200000000 & 8.087000000000 \\
\hline $\mathrm{C}$ & 0.796800000000 & -3.278300000000 & 8.608300000000 \\
\hline
\end{tabular}




$\begin{array}{cr}\mathrm{C} & 2.939700000000 \\ \mathrm{C} & 1.574900000000 \\ \mathrm{H} & -0.223000000000 \\ \mathrm{H} & 0.739900000000 \\ \mathrm{H} & 1.344600000000 \\ \mathrm{H} & 3.515100000000 \\ \mathrm{H} & 3.453400000000 \\ \mathrm{H} & 2.963700000000 \\ \mathrm{H} & 2.129500000000 \\ \mathrm{H} & 2.082100000000 \\ \mathrm{H} & 0.580300000000 \\ \mathrm{H} & -0.559300000000 \\ \mathrm{H} & -1.401700000000 \\ \mathrm{H} & 0.791600000000\end{array}$

$-2.398800000000$

$-0.968500000000$

$-3.067300000000$

$-4.043800000000$

$-3.698600000000$

$-1.535300000000$

$-2.804300000000$

$-3.166700000000$

$-1.367400000000$

$-0.060500000000$

$-0.681700000000$

1.196800000000

0.841000000000

2.049400000000
7.708600000000

9.214500000000

8.942900000000

7.827900000000

9.458800000000

7.359900000000

8.585900000000

6.928600000000

10.071000000000

8.873300000000

9.568200000000

$-1.062900000000$

$-2.630700000000$

$-1.924700000000$

3.172600000000

2.560300000000

1.269100000000

0.557000000000

1.269700000000

2.556900000000

$-0.759900000000$

1.118400000000

$-1.630200000000$

$-1.174300000000$

2.414300000000

0.028600000000

$-2.985600000000$

$-2.525900000000$

2.595200000000

3.283300000000

0.237600000000

$-3.458200000000$

$-3.716100000000$

$-2.849700000000$

1.530200000000

3.614200000000

$-0.621600000000$

1.135200000000

$-0.734600000000$

2.429300000000

0.058900000000

$-1.602800000000$

$-1.135500000000$

2.614500000000

3.300500000000

0.270900000000

$-2.931300000000$

$-2.465300000000$

1.557600000000

3.632500000000

$-0.580800000000$

$-3.389200000000$

$-3.647300000000$

$-2.775600000000$

0.627100000000

0.617400000000

$-0.960600000000$

$-4.967900000000$

$-5.692300000000$

$-5.499500000000$

$-5.304600000000$

$-5.339000000000$

$-5.531400000000$ 


\begin{tabular}{|c|c|}
\hline $\mathrm{H}$ & 1.721900000000 \\
\hline $\mathrm{H}$ & 3.567700000000 \\
\hline $\mathrm{H}$ & 2.958700000000 \\
\hline $\mathrm{H}$ & 1.831300000000 \\
\hline $\mathrm{H}$ & 4.146300000000 \\
\hline $\mathrm{H}$ & 4.851400000000 \\
\hline $\mathrm{H}$ & 3.986600000000 \\
\hline $\mathrm{C}$ & 6.672200000000 \\
\hline $\mathrm{C}$ & 6.662500000000 \\
\hline $\mathrm{C}$ & 7.782700000000 \\
\hline $\mathrm{C}$ & 7.001100000000 \\
\hline $\mathrm{H}$ & 5.886800000000 \\
\hline $\mathrm{H}$ & 6.473200000000 \\
\hline $\mathrm{H}$ & 7.629300000000 \\
\hline $\mathrm{H}$ & 7.816600000000 \\
\hline $\mathrm{H}$ & 8.759800000000 \\
\hline $\mathrm{H}$ & 7.632200000000 \\
\hline $\mathrm{H}$ & 7.972300000000 \\
\hline $\mathrm{H}$ & 7.061400000000 \\
\hline $\mathrm{H}$ & 6.257800000000 \\
\hline $\mathrm{C}$ & -2.671700000000 \\
\hline $\mathrm{C}$ & -1.399600000000 \\
\hline $\mathrm{C}$ & -2.757300000000 \\
\hline $\mathrm{C}$ & -3.883400000000 \\
\hline $\mathrm{H}$ & -1.316100000000 \\
\hline $\mathrm{H}$ & -0.493800000000 \\
\hline $\mathrm{H}$ & -1.420500000000 \\
\hline $\mathrm{H}$ & -3.658900000000 \\
\hline $\mathrm{H}$ & -2.789600000000 \\
\hline $\mathrm{H}$ & -1.895600000000 \\
\hline $\mathrm{H}$ & -3.879400000000 \\
\hline $\mathrm{H}$ & -4.827700000000 \\
\hline $\mathrm{H}$ & -3.865700000000 \\
\hline $\mathrm{C}$ & -7.084100000000 \\
\hline $\mathrm{C}$ & -6.787300000000 \\
\hline $\mathrm{C}$ & -8.275200000000 \\
\hline $\mathrm{C}$ & -7.485400000000 \\
\hline $\mathrm{H}$ & -5.945900000000 \\
\hline $\mathrm{H}$ & -6.537300000000 \\
\hline $\mathrm{H}$ & -7.661000000000 \\
\hline $\mathrm{H}$ & -8.515600000000 \\
\hline $\mathrm{H}$ & -9.163300000000 \\
\hline $\mathrm{H}$ & -8.067400000000 \\
\hline $\mathrm{H}$ & -8.371500000000 \\
\hline $\mathrm{H}$ & -7.732600000000 \\
\hline $\mathrm{H}$ & -6.692200000000 \\
\hline $\mathrm{H}$ & -2.834700000000 \\
\hline $\mathrm{H}$ & 0.835600000000 \\
\hline $\mathrm{H}$ & -1.237000000000 \\
\hline
\end{tabular}

-1.122900000000
1.634100000000
1.082000000000
1.635600000000
-1.060600000000
-0.568200000000
-2.112300000000
-2.881500000000
-4.259100000000
-2.013300000000
-3.090500000000
-4.902900000000
-4.176200000000
-4.757700000000
-1.026900000000
-2.490200000000
-1.863300000000
-3.586100000000
-2.141300000000
-3.722500000000
3.556400000000
4.342200000000
2.291500000000
4.438400000000
5.252400000000
3.751100000000
4.632700000000
1.716000000000
2.561400000000
1.634400000000
4.691200000000
3.927600000000
5.378000000000
5.410100000000
6.830200000000
4.825700000000
5.511700000000
7.273800000000
6.833500000000
7.476600000000
3.817200000000
5.453500000000
4.764300000000
6.146800000000
4.532800000000
5.958100000000
1.002200000000
-1.154200000000
-0.472300000000

Geometry data for 3 ( $\mathrm{S}_{0}$ optimization: unit $\AA$ )

$\begin{array}{lrr}\mathrm{C} & -3.176300000000 & 1.013900000000 \\ \mathrm{C} & -3.256000000000 & -0.399100000000 \\ \mathrm{C} & -2.047100000000 & -1.125400000000 \\ \mathrm{C} & -0.805500000000 & -0.467900000000 \\ \mathrm{C} & -0.818200000000 & 0.935500000000 \\ \mathrm{C} & -1.980100000000 & 1.699600000000 \\ \mathrm{~N} & 0.304200000000 & 1.643200000000 \\ \mathrm{~N} & -4.263200000000 & 1.802600000000 \\ \mathrm{C} & 1.465500000000 & 1.236200000000 \\ \mathrm{C} & 2.401100000000 & 2.301100000000 \\ \mathrm{C} & 1.753500000000 & 3.436100000000 \\ \mathrm{C} & 0.458800000000 & 3.010200000000 \\ \mathrm{C} & 2.104400000000 & 4.765900000000 \\ \mathrm{C} & 1.162400000000 & 5.676400000000\end{array}$

$-6.771900000000$

$-5.005700000000$

$-6.577200000000$

$-5.333500000000$

$-6.388100000000$

$-4.846200000000$

$-4.978700000000$

1.736200000000

1.050000000000

1.118200000000

3.219100000000

1.476600000000

$-0.024200000000$

1.180800000000

1.591400000000

1.253700000000

0.045200000000

3.313800000000

3.760800000000

3.715500000000

$-4.870900000000$

$-5.236300000000$

$-5.743900000000$

$-5.195700000000$

$-4.634100000000$

$-5.072400000000$

$-6.292500000000$

$-5.511100000000$

$-6.805300000000$

$-5.594300000000$

$-6.260600000000$

$-4.981500000000$

$-4.634300000000$

1.767400000000

1.253600000000

0.987200000000

3.243700000000

1.795200000000

0.188800000000

1.392500000000

1.338500000000

1.119400000000

$-0.084900000000$

3.340700000000

3.666500000000

3.851800000000

3.049200000000

3.053600000000

4.157300000000

$-0.338900000000$

$-0.197400000000$

$-0.056800000000$

0.128000000000

0.360200000000

0.034100000000

0.819400000000

$-0.750100000000$

1.538100000000

1.539500000000

0.905900000000

0.559900000000

0.684700000000

0.165800000000 
5.218500000000

3.882500000000

0.109200000000

$-0.002000000000$

0.994600000000

2.165900000000

3.155300000000

3.662800000000

2.602400000000

1.498700000000

3.950200000000

5.287800000000

5.821000000000

4.991300000000

2.570900000000

1.466100000000

0.426400000000

0.432900000000

7.150100000000

0.856800000000

$-0.492600000000$

0.963400000000

7.475400000000

7.452900000000

8.078100000000

7.288600000000

7.482100000000

8.226300000000

7.704200000000

1.363800000000

2.593500000000

1.243500000000

1.986500000000

$-0.550000000000$

$-6.626300000000$

$-4.968700000000$

$-5.344700000000$

$-0.637100000000$

$-1.416900000000$

1.016800000000

$-0.408700000000$

$-6.978600000000$

$-5.476000000000$

$-6.057200000000$

$-1.504600000000$

0.952700000000

$-0.438600000000$

$-5.738600000000$

$-2.753800000000$

$-3.777300000000$

$-4.193300000000$

$-3.525800000000$

$-1.517500000000$

$-0.562600000000$

0.288300000000

0.218300000000

$-2.118100000000$

$-2.490900000000$

$-1.613900000000$

$-0.738300000000$

$-1.636800000000$

$-0.614900000000$

0.284600000000

0.198400000000 $-2.992300000000$
$-0.067100000000$

0.139600000000

2.309700000000

2.968400000000

2.909200000000

2.199400000000

$-0.402100000000$

$-0.720500000000$

$-1.424200000000$

$-1.490300000000$

0.073000000000

0.364400000000

0.160700000000

$-0.414900000000$

$-2.090100000000$

$-2.870300000000$

$-2.993800000000$

$-2.333500000000$

$-0.120300000000$

3.614000000000

4.342200000000

2.563600000000

0.201900000000

$-1.618700000000$

0.737900000000

0.540500000000

2.056100000000

$-0.253100000000$

0.235100000000

$-3.611300000000$

$-3.369400000000$

$-5.133500000000$

4.658300000000

$-5.553000000000$

$-2.488200000000$

$-3.908800000000$

$-2.462100000000$

5.481800000000

3.936200000000

4.039700000000

4.094800000000

2.009700000000

3.536800000000

1.267000000000

$-4.057100000000$

$-4.006800000000$

$-4.153300000000$

2.068100000000

$-1.919700000000$

$-2.433000000000$

$-1.830500000000$

$-0.675500000000$

1.978700000000

2.994300000000

2.997500000000

2.036900000000

$-0.750800000000$

$-0.136400000000$

0.999500000000

1.027800000000

$-2.115000000000$

$-3.066600000000$

$-3.016000000000$

$-2.062900000000$

1.693600000000 
$-4.082200000000$

$-4.513200000000$

$-3.791800000000$

$-2.302200000000$

$-2.689800000000$

$-1.751000000000$

$-0.826000000000$

$-1.036600000000$

$-1.153500000000$

$-5.656700000000$

0.110400000000

3.250700000000

$-2.547800000000$

$-3.392200000000$

$-4.735100000000$

$-4.708400000000$

$-3.350600000000$

$-5.709400000000$

$-5.376800000000$

$-4.005500000000$

$-2.987200000000$

$-3.081000000000$

$-4.134900000000$

$-5.492100000000$

$-5.772900000000$

5.095000000000

5.920200000000

$-0.667800000000$

$-0.901800000000$

2.994000000000

5.929300000000

5.382500000000

3.429200000000

$-0.423500000000$

$-0.368400000000$

$-0.549900000000$

$-0.615400000000$

$-1.339700000000$

0.873900000000

1.922200000000

0.167800000000

8.526800000000

7.322100000000

6.867000000000

8.500300000000

7.280600000000

6.818600000000

7.939000000000

7.886700000000

9.129900000000

8.523300000000

6.839300000000

7.241000000000

9.273800000000

8.110700000000

8.023600000000

7.093500000000

8.747500000000

7.629400000000

2.725400000000

3.515900000000

2.469400000000

1.165300000000

0.358300000000

2.121900000000
2.139600000000

1.512000000000

0.403000000000

0.568400000000

$-0.068200000000$

$-1.145000000000$

$-1.117500000000$

$-0.046900000000$

$-0.064900000000$

$-1.678100000000$

$-3.116500000000$

0.084900000000

$-0.102400000000$

0.890800000000

0.479800000000

$-0.823400000000$

$-1.148100000000$

$-1.727900000000$

$-2.954000000000$

$-3.246800000000$

$-2.370300000000$

2.134700000000

2.945300000000

2.565300000000

1.319400000000

0.928000000000

$-0.426800000000$

2.434500000000

3.548000000000

2.180200000000

0.768100000000

$-0.636900000000$

$-2.012100000000$

$-3.633800000000$

$-2.507900000000$

4.812400000000

5.133300000000

3.652600000000

3.046800000000

2.034800000000

1.814900000000

$-0.029800000000$

1.262400000000

$-0.391600000000$

$-1.840400000000$

$-1.907700000000$

$-2.251900000000$

0.518300000000

1.806800000000

0.543300000000

2.344700000000

2.644700000000

2.336400000000

0.007600000000

$-1.332600000000$

$-0.038800000000$

0.786600000000

0.532300000000

$-0.833700000000$

$-2.306400000000$

$-3.736100000000$

$-3.902100000000$

$-5.671600000000$

$-5.381600000000$

$-5.513400000000$ 


\begin{tabular}{|c|c|c|c|}
\hline $\mathrm{H}$ & 5.401800000000 & 2.978900000000 & 4.197800000000 \\
\hline $\mathrm{H}$ & 4.664300000000 & 1.932900000000 & 5.419900000000 \\
\hline $\mathrm{H}$ & 6.420600000000 & 1.902100000000 & 5.164000000000 \\
\hline $\mathrm{H}$ & 2.979300000000 & 0.210300000000 & -5.705500000000 \\
\hline $\mathrm{H}$ & 4.507500000000 & -0.419200000000 & -6.338500000000 \\
\hline $\mathrm{H}$ & 3.286000000000 & -1.532300000000 & -5.690000000000 \\
\hline $\mathrm{H}$ & -6.307300000000 & -6.922300000000 & -1.473000000000 \\
\hline $\mathrm{H}$ & -7.150800000000 & -7.456600000000 & -2.942100000000 \\
\hline $\mathrm{H}$ & -5.675600000000 & -6.484500000000 & -3.065500000000 \\
\hline $\mathrm{H}$ & -8.488400000000 & -4.069200000000 & -3.919500000000 \\
\hline $\mathrm{H}$ & -8.429300000000 & -5.783900000000 & -4.374800000000 \\
\hline $\mathrm{H}$ & -6.985900000000 & -4.772700000000 & -4.536400000000 \\
\hline $\mathrm{H}$ & -7.001900000000 & 0.077400000000 & 5.679000000000 \\
\hline $\mathrm{H}$ & -8.555400000000 & -0.523600000000 & 6.277200000000 \\
\hline $\mathrm{H}$ & -7.385100000000 & -1.645600000000 & 5.554100000000 \\
\hline $\mathrm{H}$ & -10.132700000000 & -1.289700000000 & 2.981900000000 \\
\hline $\mathrm{H}$ & -10.341700000000 & -1.269200000000 & 4.737600000000 \\
\hline $\mathrm{H}$ & -9.256500000000 & -2.452900000000 & 3.997700000000 \\
\hline $\mathrm{H}$ & -9.813300000000 & 1.144400000000 & 4.821900000000 \\
\hline $\mathrm{H}$ & -8.290100000000 & 1.786700000000 & 4.188600000000 \\
\hline $\mathrm{H}$ & -9.529900000000 & 1.203000000000 & 3.069900000000 \\
\hline $\mathrm{H}$ & 1.941000000000 & -7.195000000000 & 0.977200000000 \\
\hline $\mathrm{H}$ & 2.753300000000 & -7.860900000000 & 2.409500000000 \\
\hline $\mathrm{H}$ & 1.323100000000 & -6.832900000000 & 2.594000000000 \\
\hline $\mathrm{H}$ & 4.236800000000 & -4.607300000000 & 3.607300000000 \\
\hline $\mathrm{H}$ & 4.101500000000 & -6.344100000000 & 3.948800000000 \\
\hline $\mathrm{H}$ & 2.703000000000 & -5.283500000000 & 4.175000000000 \\
\hline $\mathrm{H}$ & 4.934900000000 & -6.934900000000 & 1.696000000000 \\
\hline $\mathrm{H}$ & 4.214600000000 & -6.283600000000 & 0.218200000000 \\
\hline $\mathrm{H}$ & 5.153600000000 & -5.227200000000 & 1.292800000000 \\
\hline $\mathrm{H}$ & 6.035100000000 & -1.461300000000 & -3.095700000000 \\
\hline $\mathrm{H}$ & 6.253000000000 & -1.338600000000 & -4.846200000000 \\
\hline $\mathrm{H}$ & 5.113700000000 & -2.518100000000 & -4.185200000000 \\
\hline $\mathrm{H}$ & 4.334700000000 & 1.763400000000 & -4.107700000000 \\
\hline $\mathrm{H}$ & 5.542900000000 & 1.055900000000 & -3.026500000000 \\
\hline $\mathrm{H}$ & 5.830900000000 & 1.096100000000 & -4.778200000000 \\
\hline $\mathrm{H}$ & -3.740000000000 & -2.473700000000 & -2.422600000000 \\
\hline $\mathrm{H}$ & -5.103200000000 & -4.272900000000 & -3.335900000000 \\
\hline $\mathrm{H}$ & -7.997700000000 & -3.794400000000 & -0.182000000000 \\
\hline $\mathrm{H}$ & -8.322500000000 & -2.200700000000 & 1.937100000000 \\
\hline $\mathrm{H}$ & -6.159200000000 & 1.037200000000 & 3.778000000000 \\
\hline $\mathrm{H}$ & -4.404900000000 & 0.892400000000 & 2.082400000000 \\
\hline $\mathrm{H}$ & 4.180300000000 & -2.358800000000 & -2.116800000000 \\
\hline $\mathrm{H}$ & 2.172900000000 & 1.086100000000 & -3.746600000000 \\
\hline $\mathrm{H}$ & 0.407300000000 & 0.912000000000 & -2.065700000000 \\
\hline $\mathrm{H}$ & -0.424900000000 & -2.703500000000 & 2.213700000000 \\
\hline $\mathrm{H}$ & 0.850800000000 & -4.618900000000 & 3.008100000000 \\
\hline $\mathrm{H}$ & 3.775100000000 & -4.070000000000 & -0.106700000000 \\
\hline $\mathrm{H}$ & -9.287300000000 & -6.481400000000 & -2.161300000000 \\
\hline $\mathrm{H}$ & -8.536500000000 & -5.960800000000 & -0.647200000000 \\
\hline $\mathrm{H}$ & -9.427900000000 & -4.795400000000 & -1.647100000000 \\
\hline $\mathrm{H}$ & -10.218800000000 & 0.176700000000 & -2.042100000000 \\
\hline $\mathrm{H}$ & -10.971600000000 & 0.012400000000 & -3.642100000000 \\
\hline $\mathrm{H}$ & -9.441400000000 & -0.807300000000 & -3.288700000000 \\
\hline $\mathrm{H}$ & -1.614400000000 & -6.746300000000 & -1.460700000000 \\
\hline $\mathrm{H}$ & -0.223500000000 & -3.720100000000 & -4.193400000000 \\
\hline $\mathrm{H}$ & -0.866600000000 & -1.948500000000 & -2.634100000000 \\
\hline $\mathrm{H}$ & -3.269000000000 & -2.054500000000 & 2.464600000000 \\
\hline $\mathrm{H}$ & -3.996900000000 & -3.889400000000 & 3.908900000000 \\
\hline $\mathrm{H}$ & -2.747000000000 & -6.797600000000 & 0.986500000000 \\
\hline $\mathrm{C}$ & -3.925300000000 & -6.593000000000 & 3.525900000000 \\
\hline $\mathrm{C}$ & -5.437400000000 & -6.409500000000 & 3.799000000000 \\
\hline $\mathrm{C}$ & -3.713600000000 & -8.006800000000 & 2.951800000000 \\
\hline $\mathrm{C}$ & -3.150200000000 & -6.499000000000 & 4.861900000000 \\
\hline $\mathrm{H}$ & -5.655000000000 & -5.435600000000 & 4.249600000000 \\
\hline
\end{tabular}




$\begin{array}{cr}\mathrm{H} & -6.012700000000 \\ \mathrm{H} & -5.800200000000 \\ \mathrm{H} & -2.654400000000 \\ \mathrm{H} & -4.075500000000 \\ \mathrm{H} & -4.263500000000 \\ \mathrm{H} & -3.492400000000 \\ \mathrm{H} & -2.075100000000 \\ \mathrm{H} & -3.290800000000 \\ \mathrm{C} & -0.423500000000 \\ \mathrm{C} & 1.096100000000 \\ \mathrm{C} & -0.703800000000 \\ \mathrm{C} & -1.190100000000 \\ \mathrm{H} & 1.361000000000 \\ \mathrm{H} & 1.665200000000 \\ \mathrm{H} & 1.423300000000 \\ \mathrm{H} & -1.772200000000 \\ \mathrm{H} & -0.376500000000 \\ \mathrm{H} & -0.163700000000 \\ \mathrm{H} & -0.883700000000 \\ \mathrm{H} & -2.271200000000 \\ \mathrm{H} & -1.002200000000\end{array}$

Geometry data for 4 ( $\mathrm{S}_{0}$ optimization: unit $\AA$ )

$\begin{array}{lrr}\text { N } & 3.176400000000 & 10.997800000000 \\ \mathrm{~N} & 4.661300000000 & 12.420200000000 \\ \mathrm{~N} & 6.577900000000 & 8.274400000000 \\ \mathrm{~N} & 5.079800000000 & 6.826900000000 \\ \mathrm{C} & 2.285100000000 & 11.473600000000 \\ \mathrm{C} & 4.643900000000 & 11.008300000000 \\ \mathrm{C} & 5.624400000000 & 8.882600000000 \\ \mathrm{C} & 5.307200000000 & 14.606300000000 \\ \mathrm{C} & 1.795800000000 & 12.462500000000 \\ \mathrm{C} & 4.020700000000 & 10.345800000000 \\ \mathrm{C} & 2.319300000000 & 12.121500000000 \\ \mathrm{C} & 3.065400000000 & 10.562000000000 \\ \mathrm{C} & 3.589900000000 & 9.376100000000 \\ \mathrm{C} & 4.997800000000 & 8.213000000000 \\ \mathrm{C} & 5.250500000000 & 13.328300000000 \\ \mathrm{C} & 3.378000000000 & 9.201800000000 \\ \mathrm{H} & 3.775600000000 & 8.311100000000 \\ \mathrm{C} & 6.594300000000 & 8.379200000000 \\ \mathrm{C} & 4.758200000000 & 14.462500000000 \\ \mathrm{C} & 4.334400000000 & 8.975200000000 \\ \mathrm{C} & 7.814900000000 & 7.748500000000 \\ \mathrm{C} & 0.480700000000 & 14.247100000000 \\ \mathrm{C} & 4.662000000000 & 6.942500000000 \\ \mathrm{C} & 5.249100000000 & 4.880900000000 \\ \mathrm{C} & 1.862700000000 & 12.780500000000 \\ \mathrm{H} & 2.168200000000 & 12.490000000000 \\ \mathrm{C} & 5.445200000000 & 10.259200000000 \\ \mathrm{H} & 6.007900000000 & 10.785000000000 \\ \mathrm{C} & 2.672400000000 & 10.108200000000 \\ \mathrm{C} & 4.999400000000 & 6.265000000000 \\ \mathrm{C} & 4.393600000000 & 13.105000000000 \\ \mathrm{C} & 5.002500000000 & 4.769600000000 \\ \mathrm{C} & 5.755600000000 & 13.123300000000 \\ \mathrm{H} & 5.738500000000 & 12.145500000000 \\ \mathrm{C} & 0.900500000000 & 13.527000000000 \\ \mathrm{H} & 0.522000000000 & 13.767900000000 \\ \mathrm{C} & 0.965200000000 & 13.833100000000 \\ \mathrm{H} & 0.626800000000 & 14.344400000000 \\ \mathrm{C} & 4.690500000000 & 6.139900000000 \\ \mathrm{H} & 4.438800000000 & 6.601200000000 \\ \mathrm{C} & 2.106300000000 & 11.246500000000 \\ \mathrm{H} & 1.521100000000 & 11.948700000000 \\ & & \\ & & \end{array}$

2.869000000000

4.486200000000

2.762900000000

3.667000000000

2.015000000000

5.560100000000

4.700000000000

5.347700000000

$-3.983600000000$

$-4.245500000000$

$-3.501100000000$

$-5.310200000000$

$-4.632500000000$

$-3.322400000000$

$-4.981400000000$

$-3.325200000000$

$-4.263000000000$

$-2.576000000000$

$-6.056800000000$

$-5.157000000000$

$-5.733000000000$

3.991800000000

6.147200000000

7.588500000000

5.457700000000

1.928800000000

5.987800000000

6.728200000000

5.859100000000

2.872000000000

4.901100000000

4.140300000000

2.671600000000

2.148800000000

5.647600000000

5.252400000000

0.758900000000

0.293800000000

8.985200000000

7.193800000000

4.633300000000

7.183000000000

3.857900000000

3.003700000000

4.248600000000

5.280000000000

6.274800000000

6.846400000000

7.610300000000

$-0.042800000000$

4.182600000000

7.339600000000

1.838200000000

3.971400000000

3.502000000000

2.735800000000

1.748400000000

5.116900000000

6.013200000000

1.842300000000

0.895400000000

0.571200000000

$-0.016300000000$ 
4.141600000000

3.078200000000

5.757600000000

4.551100000000

7.842300000000

7.449700000000

12.642600000000

11.603400000000

7.469800000000

14.920800000000

15.510800000000

7.518200000000

7.747600000000

14.218600000000

14.049700000000

15.357200000000

16.395000000000

8.310500000000

7.871700000000

5.721500000000

6.605600000000

8.846300000000

9.231800000000

6.931400000000

6.723800000000

3.998500000000

8.805400000000

9.173200000000

15.683000000000

16.659400000000

2.513400000000

2.390900000000

2.013000000000

1.987100000000

13.556600000000

13.193400000000

6.987100000000

6.806800000000

4.065100000000

3.616500000000

3.520400000000

5.096100000000

6.676900000000

4.633100000000

5.683800000000

4.098600000000

4.588500000000

8.305600000000

15.861000000000

15.435800000000

9.896300000000

16.717000000000

4.491400000000

4.488900000000

8.609700000000

8.620300000000

8.513600000000

7.713300000000

16.341500000000

15.590000000000

17.230100000000

15.953300000000

15.126500000000

14.237000000000

15.969200000000
3.069200000000

3.112600000000

6.382600000000

5.655800000000

10.858300000000

11.218200000000

8.526300000000

8.646500000000

8.325300000000

9.453500000000

3.860100000000

5.900300000000

5.013300000000

3.297900000000

2.300400000000

8.250700000000

8.120100000000

11.755800000000

9.478600000000

7.767400000000

8.350900000000

9.858900000000

9.491000000000

8.178000000000

9.067100000000

0.502200000000

11.224300000000

11.901500000000

5.156700000000

5.632700000000

0.685700000000

1.129100000000

$-0.289200000000$

1.317600000000

9.559300000000

10.482800000000

5.787700000000

4.788500000000

$-0.153500000000$

0.497500000000

$-1.105700000000$

$-0.360000000000$

6.906800000000

$-0.449500000000$

$-0.659400000000$

$-1.407300000000$

$-0.015100000000$

13.282000000000

10.650000000000

3.770000000000

$-1.556600000000$

3.105100000000

8.415000000000

9.495500000000

$-2.069500000000$

$-1.899500000000$

$-3.148800000000$

$-1.593700000000$

1.698200000000

1.736600000000

1.203400000000

1.066000000000

4.590200000000

4.201900000000

4.539700000000 


\begin{tabular}{|c|c|c|c|}
\hline $\mathrm{H}$ & -1.542000000000 & 14.946500000000 & 5.646200000000 \\
\hline $\mathrm{C}$ & 5.523100000000 & 3.338200000000 & 6.327200000000 \\
\hline $\mathrm{H}$ & 5.647200000000 & 2.427300000000 & 5.747700000000 \\
\hline $\mathrm{C}$ & -0.920000000000 & 15.736400000000 & 2.320500000000 \\
\hline $\mathrm{H}$ & -0.061200000000 & 15.986800000000 & 1.687300000000 \\
\hline $\mathrm{H}$ & -1.602200000000 & 16.593900000000 & 2.306300000000 \\
\hline $\mathrm{H}$ & -1.446400000000 & 14.889400000000 & 1.865500000000 \\
\hline $\mathrm{C}$ & 8.142500000000 & 17.291800000000 & 3.902100000000 \\
\hline $\mathrm{H}$ & 7.844000000000 & 17.621400000000 & 4.902900000000 \\
\hline $\mathrm{H}$ & 8.571600000000 & 18.156600000000 & 3.380700000000 \\
\hline $\mathrm{H}$ & 8.931300000000 & 16.540100000000 & 4.020600000000 \\
\hline $\mathrm{C}$ & 0.188200000000 & 16.701400000000 & 4.342000000000 \\
\hline $\mathrm{H}$ & 0.482500000000 & 16.567600000000 & 5.388100000000 \\
\hline $\mathrm{H}$ & -0.494600000000 & 17.558900000000 & 4.293600000000 \\
\hline $\mathrm{H}$ & 1.090300000000 & 16.951000000000 & 3.772100000000 \\
\hline $\mathrm{C}$ & 7.209700000000 & 9.752300000000 & 13.823400000000 \\
\hline $\mathrm{H}$ & 7.979700000000 & 10.388100000000 & 13.371800000000 \\
\hline $\mathrm{H}$ & 7.352600000000 & 9.764300000000 & 14.911100000000 \\
\hline $\mathrm{H}$ & 6.236300000000 & 10.207000000000 & 13.611200000000 \\
\hline $\mathrm{C}$ & 5.476700000000 & 3.286100000000 & 7.727400000000 \\
\hline $\mathrm{C}$ & 11.806900000000 & 6.084000000000 & 6.684100000000 \\
\hline B & 4.181700000000 & 8.400800000000 & 3.191800000000 \\
\hline $\mathrm{C}$ & 5.864900000000 & 17.811400000000 & 2.948500000000 \\
\hline $\mathrm{H}$ & 5.007700000000 & 17.434400000000 & 2.378900000000 \\
\hline $\mathrm{H}$ & 6.271700000000 & 18.681100000000 & 2.417300000000 \\
\hline $\mathrm{H}$ & 5.493200000000 & 18.157700000000 & 3.918600000000 \\
\hline $\mathrm{C}$ & 8.675200000000 & 7.734500000000 & 13.681100000000 \\
\hline $\mathrm{H}$ & 8.795600000000 & 6.695300000000 & 13.354200000000 \\
\hline $\mathrm{H}$ & 8.779700000000 & 7.751400000000 & 14.771900000000 \\
\hline $\mathrm{H}$ & 9.498800000000 & 8.323300000000 & 13.261500000000 \\
\hline $\mathrm{C}$ & 6.209800000000 & 7.438800000000 & 13.954100000000 \\
\hline $\mathrm{H}$ & 5.202900000000 & 7.815400000000 & 13.745100000000 \\
\hline $\mathrm{H}$ & 6.342700000000 & 7.431100000000 & 15.043100000000 \\
\hline $\mathrm{H}$ & 6.258900000000 & 6.403200000000 & 13.598200000000 \\
\hline $\mathrm{C}$ & 0.957600000000 & 9.799600000000 & -1.870200000000 \\
\hline $\mathrm{H}$ & 0.501700000000 & 8.954300000000 & -1.342300000000 \\
\hline $\mathrm{H}$ & 0.798500000000 & 9.655300000000 & -2.946000000000 \\
\hline $\mathrm{H}$ & 0.419200000000 & 10.706400000000 & -1.574700000000 \\
\hline $\mathrm{C}$ & 3.076700000000 & 11.092700000000 & -2.328200000000 \\
\hline $\mathrm{H}$ & 2.611000000000 & 12.041700000000 & -2.042300000000 \\
\hline $\mathrm{H}$ & 2.933700000000 & 10.964100000000 & -3.408200000000 \\
\hline $\mathrm{H}$ & 4.152300000000 & 11.177800000000 & -2.135200000000 \\
\hline $\mathrm{C}$ & 12.661800000000 & 7.069500000000 & 5.852100000000 \\
\hline $\mathrm{H}$ & 12.214100000000 & 7.273100000000 & 4.873600000000 \\
\hline $\mathrm{H}$ & 13.663900000000 & 6.657000000000 & 5.680200000000 \\
\hline $\mathrm{H}$ & 12.772700000000 & 8.027200000000 & 6.373400000000 \\
\hline $\mathrm{C}$ & 7.029300000000 & 1.336300000000 & 8.093600000000 \\
\hline $\mathrm{H}$ & 7.136400000000 & 1.172100000000 & 7.016100000000 \\
\hline $\mathrm{H}$ & 7.167700000000 & 0.369800000000 & 8.594100000000 \\
\hline $\mathrm{H}$ & 7.838700000000 & 2.003600000000 & 8.410800000000 \\
\hline $\mathrm{C}$ & 11.687500000000 & 4.742300000000 & 5.922500000000 \\
\hline $\mathrm{H}$ & 11.094300000000 & 4.019900000000 & 6.494700000000 \\
\hline $\mathrm{H}$ & 12.680900000000 & 4.308900000000 & 5.751300000000 \\
\hline $\mathrm{H}$ & 11.206700000000 & 4.868600000000 & 4.946800000000 \\
\hline $\mathrm{C}$ & 12.545300000000 & 5.815300000000 & 8.009100000000 \\
\hline $\mathrm{H}$ & 12.692400000000 & 6.734600000000 & 8.587500000000 \\
\hline $\mathrm{H}$ & 13.535600000000 & 5.394000000000 & 7.801600000000 \\
\hline $\mathrm{H}$ & 12.006200000000 & 5.097500000000 & 8.637800000000 \\
\hline $\mathrm{C}$ & 5.568400000000 & 2.074600000000 & 9.981600000000 \\
\hline $\mathrm{H}$ & 6.346700000000 & 2.740300000000 & 10.371400000000 \\
\hline $\mathrm{H}$ & 5.708000000000 & 1.092900000000 & 10.448200000000 \\
\hline $\mathrm{H}$ & 4.594800000000 & 2.458400000000 & 10.307200000000 \\
\hline $\mathrm{C}$ & 4.542500000000 & 15.353000000000 & 11.877100000000 \\
\hline $\mathrm{H}$ & 4.241600000000 & 14.341000000000 & 12.167800000000 \\
\hline $\mathrm{H}$ & 4.376800000000 & 16.010300000000 & 12.739 \\
\hline
\end{tabular}




$\begin{array}{ll}\mathrm{H} & 5.617800000000 \\ \mathrm{C} & 5.648000000000 \\ \mathrm{C} & 4.196300000000 \\ \mathrm{H} & 5.268000000000 \\ \mathrm{H} & 4.006200000000 \\ \mathrm{H} & 3.647900000000 \\ \mathrm{C} & 2.240000000000 \\ \mathrm{H} & 1.652400000000 \\ \mathrm{H} & 2.053400000000 \\ \mathrm{C} & 4.535600000000 \\ \mathrm{H} & 3.543700000000 \\ \mathrm{H} & 4.649200000000 \\ \mathrm{H} & 4.564600000000 \\ \mathrm{H} & 1.862100000000\end{array}$

Geometry data for 5 ( $\mathrm{S}_{0}$ optimization: unit $\AA$ )

$\begin{array}{lrr}\mathrm{C} & -3.896400000000 & 0.776400000000 \\ \mathrm{C} & -4.108300000000 & -0.616300000000 \\ \mathrm{C} & -2.980000000000 & -1.317300000000 \\ \mathrm{C} & -1.680800000000 & -0.776500000000 \\ \mathrm{C} & -1.468900000000 & 0.616200000000 \\ \mathrm{C} & -2.597200000000 & 1.317200000000 \\ \mathrm{~N} & -2.964500000000 & -2.686200000000 \\ \mathrm{C} & -1.625400000000 & -3.073000000000 \\ \mathrm{C} & -0.807700000000 & -1.916700000000 \\ \mathrm{~N} & -2.612700000000 & 2.686100000000 \\ \mathrm{C} & -3.951800000000 & 3.072900000000 \\ \mathrm{C} & -4.769500000000 & 1.916500000000 \\ \mathrm{C} & -1.051500000000 & -4.344800000000 \\ \mathrm{C} & 0.336000000000 & -4.441900000000 \\ \mathrm{C} & 1.184900000000 & -3.315500000000 \\ \mathrm{C} & 0.588500000000 & -2.053200000000 \\ \mathrm{C} & -4.525700000000 & 4.344700000000 \\ \mathrm{C} & -5.913100000000 & 4.441800000000 \\ \mathrm{C} & -6.762100000000 & 3.315400000000 \\ \mathrm{C} & -6.165700000000 & 2.053100000000 \\ \mathrm{C} & -4.136400000000 & -3.445800000000 \\ \mathrm{C} & -1.440800000000 & 3.445700000000 \\ \mathrm{C} & -5.305900000000 & -2.929300000000 \\ \mathrm{C} & -6.469000000000 & -3.757100000000 \\ \mathrm{C} & -6.463600000000 & -5.010600000000 \\ \mathrm{C} & -5.322900000000 & -5.427700000000 \\ \mathrm{C} & -4.160800000000 & -4.663300000000 \\ \mathrm{~N} & -7.586600000000 & -3.289100000000 \\ \mathrm{C} & -0.271300000000 & 2.929100000000 \\ \mathrm{C} & 0.891800000000 & 3.757000000000 \\ \mathrm{C} & 0.886400000000 & 5.010400000000 \\ \mathrm{C} & -0.254300000000 & 5.427600000000 \\ \mathrm{C} & -1.416400000000 & 4.663200000000 \\ \mathrm{C} & -7.459800000000 & -2.240700000000 \\ \mathrm{C} & -8.576100000000 & -2.206800000000 \\ \mathrm{C} & -9.487000000000 & -3.237500000000 \\ \mathrm{C} & -8.864700000000 & -3.871200000000 \\ \mathrm{C} & -6.396300000000 & -1.333600000000 \\ \mathrm{C} & -6.456500000000 & -0.426600000000 \\ \mathrm{C} & -7.504600000000 & -0.403400000000 \\ \mathrm{C} & -8.583800000000 & -1.295700000000 \\ \mathrm{C} & -10.764100000000 & -3.617300000000 \\ \mathrm{C} & -1.469300000000 & -4.616100000000 \\ \mathrm{C} & -9.566900000000 & -5.191100000000 \\ \mathrm{C} & 1.882600000000 & 3.2890000000000 \\ \mathrm{C} & 2.240600000000 \\ \mathrm{C} & 2.966700000000 \\ \mathrm{C} & & 3.237400000000 \\ \mathrm{C} & & \end{array}$

11.666000000000

8.449300000000

10.358300000000

10.136600000000

11.234800000000

9.514600000000

10.993500000000

10.145100000000

11.849600000000

7.997800000000

8.243100000000

8.500400000000

6.918100000000

11.252800000000

$-0.407100000000$

$-0.272200000000$

0.204900000000

0.407300000000

0.272400000000

$-0.204600000000$

0.487300000000

0.687200000000

0.649400000000

$-0.487100000000$

$-0.687000000000$

$-0.649100000000$

0.757300000000

0.788400000000

0.737400000000

0.666800000000

$-0.757100000000$

$-0.788200000000$

$-0.737200000000$

$-0.666600000000$

0.588900000000

$-0.588700000000$

$-0.035500000000$

$-0.050000000000$

0.577100000000

1.256700000000

1.281200000000

$-0.760800000000$

0.035700000000

0.050200000000

$-0.576900000000$

$-1.256500000000$

$-1.280900000000$

$-1.674000000000$

$-2.533100000000$

$-2.064800000000$

$-0.956700000000$

$-1.748100000000$

$-2.831700000000$

$-3.765200000000$

$-3.582500000000$

$-2.470100000000$

$-1.782500000000$

$-0.659900000000$

$-0.231600000000$

0.761000000000

1.674200000000

2.533300000000

2.065000000000 


\begin{tabular}{|c|c|c|c|}
\hline $\mathrm{C}$ & 3.287500000000 & 3.871100000000 & 0.957000000000 \\
\hline $\mathrm{C}$ & 0.819100000000 & 1.333500000000 & 1.748300000000 \\
\hline $\mathrm{C}$ & 0.879300000000 & 0.426500000000 & 2.832000000000 \\
\hline $\mathrm{C}$ & 1.927300000000 & 0.403300000000 & 3.765400000000 \\
\hline $\mathrm{C}$ & 3.006500000000 & 1.295600000000 & 3.582700000000 \\
\hline $\mathrm{C}$ & 5.186900000000 & 3.617200000000 & 2.470400000000 \\
\hline $\mathrm{C}$ & 5.892100000000 & 4.616000000000 & 1.782700000000 \\
\hline $\mathrm{C}$ & 5.271500000000 & 5.191000000000 & 0.660200000000 \\
\hline $\mathrm{C}$ & 3.989700000000 & 4.832100000000 & 0.231900000000 \\
\hline B & -5.289500000000 & -1.533400000000 & -0.689800000000 \\
\hline B & -0.287700000000 & 1.533300000000 & 0.690000000000 \\
\hline $\mathrm{H}$ & -1.649700000000 & -5.245900000000 & 0.723900000000 \\
\hline $\mathrm{H}$ & 0.770300000000 & -5.436900000000 & 0.818800000000 \\
\hline $\mathrm{H}$ & 1.197600000000 & -1.162700000000 & 0.591500000000 \\
\hline $\mathrm{H}$ & -3.927500000000 & 5.245800000000 & -0.723700000000 \\
\hline $\mathrm{H}$ & -6.347500000000 & 5.436800000000 & -0.818600000000 \\
\hline $\mathrm{H}$ & -6.774800000000 & 1.162600000000 & -0.591300000000 \\
\hline $\mathrm{H}$ & -7.310500000000 & -5.675800000000 & 0.507900000000 \\
\hline $\mathrm{H}$ & -5.336900000000 & -6.385200000000 & 1.770700000000 \\
\hline $\mathrm{H}$ & -3.304400000000 & -4.998500000000 & 1.849900000000 \\
\hline $\mathrm{H}$ & 1.733300000000 & 5.675700000000 & -0.507600000000 \\
\hline $\mathrm{H}$ & -0.240300000000 & 6.385100000000 & -1.770500000000 \\
\hline $\mathrm{H}$ & -2.272800000000 & 4.998400000000 & -1.849700000000 \\
\hline $\mathrm{H}$ & -5.636400000000 & 0.272200000000 & -2.941000000000 \\
\hline $\mathrm{H}$ & -9.418700000000 & -1.289200000000 & -4.277600000000 \\
\hline $\mathrm{H}$ & -11.215200000000 & -3.116800000000 & -3.322700000000 \\
\hline $\mathrm{H}$ & -11.373700000000 & -5.941000000000 & -0.079400000000 \\
\hline $\mathrm{H}$ & -9.173500000000 & -5.276200000000 & 0.673000000000 \\
\hline $\mathrm{H}$ & 0.059100000000 & -0.272300000000 & 2.941200000000 \\
\hline $\mathrm{H}$ & 3.841500000000 & 1.289100000000 & 4.277900000000 \\
\hline $\mathrm{H}$ & 5.638000000000 & 3.116700000000 & 3.323000000000 \\
\hline $\mathrm{H}$ & 5.796600000000 & 5.940800000000 & 0.079700000000 \\
\hline $\mathrm{H}$ & 3.596300000000 & 5.276100000000 & -0.672800000000 \\
\hline $\mathrm{C}$ & 7.299900000000 & 5.024100000000 & 2.256800000000 \\
\hline $\mathrm{C}$ & 7.220200000000 & 5.549500000000 & 3.710000000000 \\
\hline $\mathrm{C}$ & 8.239700000000 & 3.795900000000 & 2.208100000000 \\
\hline $\mathrm{C}$ & 7.916400000000 & 6.132100000000 & 1.381800000000 \\
\hline $\mathrm{H}$ & 6.567900000000 & 6.427900000000 & 3.773700000000 \\
\hline $\mathrm{H}$ & 6.826700000000 & 4.792000000000 & 4.396000000000 \\
\hline $\mathrm{H}$ & 8.216200000000 & 5.838200000000 & 4.067900000000 \\
\hline $\mathrm{H}$ & 8.321000000000 & 3.405300000000 & 1.187300000000 \\
\hline $\mathrm{H}$ & 9.245700000000 & 4.070400000000 & 2.548900000000 \\
\hline $\mathrm{H}$ & 7.882200000000 & 2.982800000000 & 2.848600000000 \\
\hline $\mathrm{H}$ & 8.909800000000 & 6.393400000000 & 1.763700000000 \\
\hline $\mathrm{H}$ & 8.038200000000 & 5.810900000000 & 0.341000000000 \\
\hline $\mathrm{H}$ & 7.309100000000 & 7.044400000000 & 1.390400000000 \\
\hline $\mathrm{C}$ & 1.918100000000 & -0.521700000000 & 4.999900000000 \\
\hline $\mathrm{C}$ & 0.756400000000 & -1.533600000000 & 4.966800000000 \\
\hline $\mathrm{C}$ & 3.241000000000 & -1.317400000000 & 5.092000000000 \\
\hline $\mathrm{C}$ & 1.766100000000 & 0.349400000000 & 6.270900000000 \\
\hline $\mathrm{H}$ & -0.219700000000 & -1.036600000000 & 4.989500000000 \\
\hline $\mathrm{H}$ & 0.793900000000 & -2.169200000000 & 4.075200000000 \\
\hline $\mathrm{H}$ & 0.811600000000 & -2.185300000000 & 5.846300000000 \\
\hline $\mathrm{H}$ & 4.113700000000 & -0.663100000000 & 5.188600000000 \\
\hline $\mathrm{H}$ & 3.226600000000 & -1.974100000000 & 5.970300000000 \\
\hline $\mathrm{H}$ & 3.389500000000 & -1.942300000000 & 4.204600000000 \\
\hline $\mathrm{H}$ & 1.757900000000 & -0.279900000000 & 7.169700000000 \\
\hline $\mathrm{H}$ & 2.589300000000 & 1.065700000000 & 6.370300000000 \\
\hline $\mathrm{H}$ & 0.829900000000 & 0.918600000000 & 6.245300000000 \\
\hline $\mathrm{C}$ & -12.877100000000 & -5.024200000000 & -2.256500000000 \\
\hline $\mathrm{C}$ & -13.816900000000 & -3.796000000000 & -2.207800000000 \\
\hline $\mathrm{C}$ & -12.797400000000 & -5.549600000000 & -3.709700000000 \\
\hline $\mathrm{C}$ & -13.493600000000 & -6.132200000000 & -1.381500000000 \\
\hline $\mathrm{H}$ & -13.898200000000 & -3.405400000000 & -1.187000000000 \\
\hline $\mathrm{H}$ & 00000000 & -2.982900000000 & \\
\hline
\end{tabular}


$-4.070500000000$

$-6.428000000000$

$-5.838300000000$

$-4.792100000000$

$-6.393500000000$

$-7.044500000000$

$-5.811000000000$

0.521700000000

1.317300000000

1.533500000000

$-0.349500000000$

0.663000000000

1.942200000000

1.974000000000

1.036600000000

2.185300000000

2.169100000000

0.279900000000

$-0.918600000000$

$-1.065800000000$

3.521200000000

4.336600000000

2.187500000000

4.299400000000

5.325600000000

3.817100000000

4.486700000000

1.592000000000

2.384900000000

1.577100000000

4.459800000000

3.743500000000

5.281000000000

$-3.521300000000$

$-2.187600000000$

$-4.336700000000$

$-4.299600000000$

$-1.592100000000$

$-1.577200000000$

$-2.385100000000$

$-5.325700000000$

$-4.486800000000$

$-3.817200000000$

$-4.459900000000$

$-5.281200000000$

$-3.743700000000$
$-2.548600000000$

$-3.773500000000$

$-4.067600000000$

$-4.395800000000$

$-1.763400000000$

$-1.390100000000$

$-0.340700000000$

$-4.999600000000$

$-5.091700000000$

$-4.966500000000$

$-6.270600000000$

$-5.188300000000$

$-4.204300000000$

$-5.969900000000$

$-4.989300000000$

$-5.846000000000$

$-4.074900000000$

$-7.169400000000$

$-6.245100000000$

$-6.370000000000$

$-0.694200000000$

$-1.927000000000$

$-0.685100000000$

0.591400000000

$-1.961800000000$

$-2.858400000000$

$-1.904100000000$

0.206000000000

$-0.679400000000$

$-1.566600000000$

0.641100000000

1.485800000000

0.628400000000

0.694400000000

0.685200000000

1.927200000000

$-0.591200000000$

$-0.205800000000$

1.566800000000

0.679600000000

1.962000000000

1.904300000000

2.858600000000

$-0.641000000000$

$-0.628200000000$

$-1.485700000000$ 